\title{
BOUNDARY VALUE PROBLEMS FOR THE INVISCID PRIMITIVE EQUATIONS IN LIMITED DOMAINS
}

\author{
A. ROUSSEAU ${ }^{b}$, R. TEMAM* $*$ AND J. TRIBBIA ${ }^{\sharp}$ \\ Date: January 10, 2008 \\ bInstitut National de Recherche en Informatique et Automatique (INRIA), France. \\ ${ }^{*}$ The Institute for Scientific Computing and Applied Mathematics, \\ Indiana University, Bloomington, IN, USA. \\ ${ }^{\sharp}$ National Center for Atmospheric Research, Boulder, Colorado, USA.
}

\section{Contents}

Abstract 2

1. Introduction 2

1.1. The Inviscid Primitive Equations 4

1.2. Normal Modes Expansion 8

1.3. Position of the Problem 12

2. Two Dimensionnal $x-z$ Case $\quad 12$

2.1. Linear and Nonlinear 2D Primitive Equations 13

2.2. The Modal Equations and Boundary Conditions 14

2.3. Well-Posedness Results 17

2.4. Numerical Simulations 32

2.5. Numerical Results 36

3. Space Dimension 2.5 44

3.1. Motivations 44

3.2. The 2.5D Primitive Equations 46

3.3. Well-Posedness of the Linear System 52

3.4. Special Case of Impenetrable Boundaries 69

4. The Full 3D Linear Case 75

4.1. Equations and Preliminary Results $\quad 75$

4.2. Subcritical Modes 84

4.3. Supercritical Modes 90

4.4. The initial and boundary value problem for the full system 98

5. Conclusion 105

$\begin{array}{ll}\text { References } & 106\end{array}$ 


\section{Abstract}

This work aims to contribute to what is considered as a major computational issue for the geophysical fluid dynamics (GFD) for the coming years, that is the boundary conditions for numerical computations in a limited domain, with a boundary that has (at least partly) no physical justification. Numerical computations in limited domains in ocean and atmosphere are "constantly" required (and sometimes lead to commercial softwares) in order to provide forecasts for agriculture, tourism industry, insurances, aircraft navigation, etc. This article focuses on the nonviscous primitive equations in a limited domain, in space dimension 2, 2.5 and 3 and provides in each case a set of boundary conditions wich is shown to lead to a well-posed problem. The suitability of these new boundary conditions is also computationnally evidenced in space dimension two.

\section{INTRODUCTION}

Limited area models (LAM) are constantly used for numerical simulations of geophysical flows. Indeed when refined information are needed, it would be far too costly and in fact not computationnally feasible at all, to perform simulations for the whole atmosphere, or for the whole ocean. Numerical computations in limited domains in ocean and atmosphere are hence "constantly" required (and sometimes lead to commercial softwares) in order to provide forecasts for agriculture, tourism industry, insurances, aircraft navigation, etc. LAM are used in an essential way for strongly perturbed systems such as tropical storms, squall lines or mid-latitude cyclones, to overcome the error due to the parametrization schemes and numerical truncation errors introduced purely by insufficient model resolutions for large areas or global models.

The penalty for using a limited area model is the appearance of a domain with nonphysical boundaries where no physical law will provide natural boundary conditions. Hence beside the usual difficulty of writing boundary conditions on top or bottom of the ocean or atmosphere, now appears the difficulty of writing boundary conditions on the nonphysical lateral boundary. Such a difficulty has been known since early works of J. von Neumann and J.G. Charney and various remedies have been proposed and implemented over the years; see e.g. Charney, Fjörtoft, and von Neumann (1950), Bennett and Kloeden (1978), Bennett and Chua (1999). However it is expected that, for the 
high resolution models which will be used in coming years with the increase of computing powers and computer memory, the remedies which have been used will lead to spurious modes which will damage the whole computation; see for example in Temam and Tribbia (2003, Figures 3 and 4), the effect of spurious modes and their resolution. The tutorial article by Warner, Peterson, and Treadon (1997) describes the motivations and the computational difficulties for this problem. The present work aims to contribute to what is considered as a major computational issue for the geophysical fluid dynamics (GFD) for the coming years, that is the boundary conditions for numerical computations in a limited domain, with a boundary that has (at least partly) no physical justification.

Beside the computational difficulty, a mathematical difficulty arises. Indeed, as we explain below, for the equations that we consider (the primitive equations without viscosity), there is no set of boundary conditions which produces a well-posed problem. Hence, for the boundary conditions that we propose, we need also to address questions of wellposedness, which we do in the context of the linearized equations.

The equations concerned by this kind of applications are well-known: primitive equations (PEs) and shallow water equations (SWEs). This work is fully dedicated to the PEs, that we will recall later on. More precisely, we only consider inviscid PEs since the viscosity effects mainly appear after a few days, that is beyond the forecast period that we are interested in. For the case of viscous PEs, for which the mathematical aspects have been widely studied from the initial works of Lions, Temam, and Wang (1992a,b) to the most recent results of Cao and Titi (2007), Kobelkov (2006), Kukavica and Ziane (2007), and numerous authors in between, the reader is referred to the review papers Temam and Ziane (2004) and its updated form Petcu, Temam, and Ziane (2008) in this volume. To the best of our knowledge, the inviscid case has been left unexplored for years since the negative result of Oliger and Sundström (1978), that showed that inviscid PEs could not be wellposed for any set of boundary conditions of local type. Recent needs in geophysical fluid dynamics put these problems back on the frontstage, and the issue of open boundary conditions has been recently studied in Temam and Tribbia (2003), Rousseau, Temam, and Tribbia (2005b, 2007), Blayo and Debreu (2005). The use of such boundary conditions is now taken into account in realistic numerical simulations, at least for the so-called barotropic mode (see e.g. Madec, Delecluse, Imbard, and Lévy (1998), Dumas and Lazure (2007)). 
We focus our study on the linearized PEs, since the boundary condition difficulty is already fully present in the linear case. However, the numerical simulations presented in the sequel relate as well to nonlinear simulations. They seem to indicate that the nonlinear system behaves like the linear one, at least for sufficiently small times and initial data, as far as the boundary conditions are concerned.

Before recalling the equations and the negative result Oliger and Sundström (1978) quoted above, let us draw the outline of this article. In Section 2, we both provide the mathematical and numerical results obtained in Rousseau, Temam, and Tribbia (2005b, 2007), and that concern the two-dimensional $(x-z)$ case. Then, before going into the full 3D model, we propose in Section 3 a simplified 2.5D model, in view of performing (in dimension two) computations of physical significance ; see the introduction to Section 3 for a detailed description of the motivations for this model. Finally, we end this overview with the most recent mathematical results on the full three dimensional case presented in Section 4.

In summary our contributions in this article are as follows:

(1) In space dimension 2, 2.5 and 3 we propose sets of boundary conditions which lead to well-posed initial and boundary value problems for the linearized primitive equations without viscosity.

(2) Numerical simulations performed in space dimension two support the conjecture that the nonlinear analog of these boundary conditions produce a well-posed nonlinear problem.

(3) Numerical simulations performed in space dimension two show that the boundary conditions that we propose satisfactorily solve the problem of lateral boundary conditions for the limited area models (LAM), with a precision of a few percents.

The rest of this section is devoted to describing the primitive equations, their linearization around a stratified state, and the normal mode expansion in the vertical direction.

1.1. The Inviscid Primitive Equations. We now recall the Primitive Equations (PEs); the emphasis will be on the case of the ocean. The case of the atmosphere can be studied similarly with minor changes, as well as the coupled atmosphere and ocean; see e.g. the article Petcu, Temam, and Ziane (2008) in this volume. The equations are derived from the Boussinesq equations by making the hydrostatic assumption 
which amounts to replacing the conservation of momentum in the vertical direction by the hydrostatic equation. Hence the equations

$$
\begin{array}{r}
\frac{\partial \widetilde{\mathbf{v}}}{\partial t}+(\widetilde{\mathbf{v}} \cdot \nabla) \widetilde{\mathbf{v}}+\widetilde{w} \frac{\partial \widetilde{\mathbf{v}}}{\partial z}+f k \times \widetilde{\mathbf{v}}+\frac{1}{\rho_{0}} \nabla \widetilde{p}=F_{\widetilde{\mathbf{v}}} \\
\frac{\partial \widetilde{p}}{\partial z}=-\widetilde{\rho} g, \\
\nabla \widetilde{\mathbf{v}}+\frac{\partial \widetilde{w}}{\partial z}=0, \\
\frac{\partial \widetilde{T}}{\partial t}+(\widetilde{\mathbf{v}} \cdot \nabla) \widetilde{T}+\widetilde{w} \frac{\partial \widetilde{T}}{\partial z}=Q_{\widetilde{T}}, \\
\widetilde{\rho}=\rho_{0}\left(1-\alpha\left(\widetilde{T}-T_{0}\right)\right) .
\end{array}
$$

In these equations $\widetilde{\mathbf{v}}=(\widetilde{u}, \widetilde{v})$ is the horizontal velocity, $\widetilde{w}$ the vertical velocity, $\widetilde{p}$ the pressure, $\widetilde{\rho}$ the density, and $\widetilde{T}$ the temperature; $g$ is the gravitational acceleration, and $f$ the Coriolis parameter. The horizontal gradient is denoted by $\nabla$. Equation (1.1e) is the equation of state of the fluid, $\rho_{0}$ and $T_{0}$ are constant reference values of $\widetilde{\rho}$ and $\widetilde{T}$, and $\alpha>0$ is constant; this equation of state is linear, although more involved nonlinear state equations could be considered.

Equation $(1.1 \mathrm{~b})$ is the so-called hydrostatic equation. The other equations correspond to the Boussinesq approximation (see e.g. Pedlosky (1987), Washington and Parkinson (1986) and Salmon (1998) for more details).

In the physical context the forcing terms $F_{\widetilde{\mathbf{v}}}=\left(F_{\widetilde{u}}, F_{\widetilde{v}}\right)$ and $Q_{\widetilde{T}}$ do not exist, but we introduce them here for mathematical generality and to study the case of nonhomogeneous boundary conditions by homogenization of the boundary contitions.

We now consider a reference stratified flow with constant velocity $\overline{\mathbf{v}}_{0}=\left(\bar{U}_{0}, 0\right)=\bar{U}_{0} e_{x}$, and density, temperature and pressure of the 
form $\rho_{0}+\bar{\rho}, T_{0}+\bar{T}, p_{0}+\bar{p}$ with $d \bar{p} / d z$ constant and thus

$$
\begin{aligned}
\bar{T}(z) & =\frac{N^{2}}{\alpha g} z \\
\bar{\rho}(z) & =-\rho_{0} \alpha \bar{T}(z)=-\frac{\rho_{0} N^{2}}{g} z \\
\frac{d \bar{T}}{d z}(z) & =\frac{N^{2}}{\alpha g} \\
\frac{d \bar{\rho}}{d z}(z) & =-\frac{\rho_{0}}{g} N^{2} \\
\frac{d \bar{p}}{d z}(z) & =-\left(\rho_{0}+\bar{\rho}\right) g .
\end{aligned}
$$

Here $N$ is the buoyancy frequency or Brunt-Väisälä frequency, assumed to be constant.

We then decompose the unknown functions $\widetilde{\mathbf{v}}, \widetilde{\rho}, \widetilde{T}, \widetilde{p}$ in the following way:

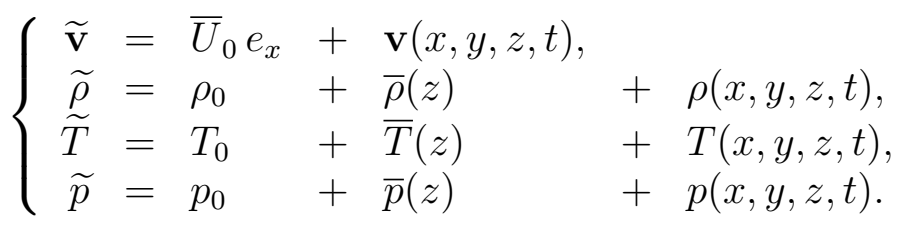

Equations (1.1b), (1.1d) and (1.1e) become

$$
\begin{aligned}
& \frac{\partial p}{\partial z}=-\rho g \\
& \frac{\partial T}{\partial t}+(\widetilde{\mathbf{v}} \cdot \nabla) T+w \frac{\partial T}{\partial z}+\frac{N^{2}}{\alpha g} w=F_{T}, \\
& \rho=-\rho_{0} \alpha T .
\end{aligned}
$$


We infer from (1.1) and (1.8)-(1.10) the following equations for $u, v$, $w, \phi=p / \rho_{0}$ and $\psi=\phi_{z}=\alpha g T$ :

$$
\frac{\partial u}{\partial t}+\bar{U}_{0} \frac{\partial u}{\partial x}+u \frac{\partial u}{\partial x}+v \frac{\partial u}{\partial y}+w \frac{\partial u}{\partial z}
$$

$$
-f v+\frac{\partial \phi}{\partial x}=F_{u}
$$

$$
\frac{\partial v}{\partial t}+\bar{U}_{0} \frac{\partial v}{\partial x}+u \frac{\partial v}{\partial x}+v \frac{\partial v}{\partial y}+w \frac{\partial v}{\partial z}
$$

$$
+f u+\frac{\partial \phi}{\partial y}=F_{v}-f \bar{U}_{0},
$$

$\frac{\partial \psi}{\partial t}+\bar{U}_{0} \frac{\partial \psi}{\partial x}+u \frac{\partial \psi}{\partial x}+v \frac{\partial \psi}{\partial y}+w \frac{\partial \psi}{\partial z}+N^{2} w=F_{\psi}$,

$$
\frac{\partial \phi}{\partial z}=-\frac{\rho}{\rho_{0}} g=\psi
$$

$$
\frac{\partial u}{\partial x}+\frac{\partial v}{\partial y}+\frac{\partial w}{\partial z}=0
$$

From equation (1.11e)we find:

$$
w(x, y, z)=\int_{z}^{0}\left(\frac{\partial u}{\partial x}\left(x, y, z^{\prime}\right)+\frac{\partial v}{\partial y}\left(x, y, z^{\prime}\right)\right) d z^{\prime},
$$

which makes the vertical velocity $w$ a diagnostic variable, whereas it is a prognostic one in the Navier Stokes Equations.

The PEs (1.11a)-(1.11e), linearized around the stratified flow $\overline{\mathbf{v}}_{0}=$ $\bar{U}_{0} e_{x}, \bar{\rho}, \bar{T}, \bar{p}$, read:

$$
\begin{aligned}
\frac{\partial u}{\partial t}+\bar{U}_{0} \frac{\partial u}{\partial x}-f v+\frac{\partial \phi}{\partial x} & =F_{u}, \\
\frac{\partial v}{\partial t}+\bar{U}_{0} \frac{\partial v}{\partial x}+f u+\frac{\partial \phi}{\partial y} & =F_{v}-f \bar{U}_{0}, \\
\frac{\partial \psi}{\partial t}+\bar{U}_{0} \frac{\partial \psi}{\partial x}+N^{2} w & =F_{\psi}, \\
\frac{\partial \phi}{\partial z}=-\frac{\rho}{\rho_{0}} g & =\psi, \\
\frac{\partial u}{\partial x}+\frac{\partial v}{\partial y}+\frac{\partial w}{\partial z} & =0 .
\end{aligned}
$$

We will consider the flow in the three-dimensional domain $\mathcal{M}=$ $\mathcal{M}^{\prime} \times\left(-L_{3}, 0\right)$ where $\mathcal{M}^{\prime}$ is the interface atmosphere/ocean, $\mathcal{M}^{\prime}=$ $\left(0, L_{1}\right) \times\left(0, L_{2}\right)$. Naturally, we supplement equation (1.13) with the 
following top and bottom boundary conditions (just imposed by kinematics):

$$
w\left(x, y, z=-L_{3}, t\right)=w(x, y, z=0, t)=0, \quad \forall(x, y) \in \mathcal{M}^{\prime}, t>0 .
$$

The aim of this work is to introduce some lateral boundary conditions at $x=0, L_{1}$, and $y=0, L_{2}$, that are both physically reasonable and computationnally satisfying $^{1}$, and that lead to the well-posedness of the problem (1.13).

\subsection{Normal Modes Expansion.}

The first step of the analysis of (1.13) consists, by separation of variables, in looking for solutions of the form

$$
\left\{\begin{array}{l}
u(x, y, z, t)=\mathcal{U}(z) \hat{u}(x, y, t), v(x, y, z, t)=\mathcal{V}(z) \hat{v}(x, y, t) \\
\psi(x, y, z, t)=\Psi(z) \hat{\psi}(x, y, t) \\
w(x, y, z, t)=\mathcal{W}(z) \hat{w}(x, y, t), \phi(x, y, z, t)=\Phi(z) \hat{\phi}(x, y, t) .
\end{array}\right.
$$

Substituting these expressions into (1.13), we find that $\mathcal{U}, \mathcal{V}, \Phi$ must be proportional and $\mathcal{W}$ proportional to $\Psi$. So we just take $\mathcal{V}=\Phi=\mathcal{U}$, and $\Psi=\mathcal{W}$. Indeed the third equation (1.13) implies that

$$
-\frac{\hat{\psi}_{t}+\bar{U}_{0} \hat{\psi}_{x}}{N^{2} \hat{w}}=\frac{\mathcal{W}}{\Psi}\left(=c_{1}^{\prime}\right)
$$

and these quantities are constant since the left-hand side of the last equation depends ${ }^{2}$ on $x, y$ and $t$ and the right-hand side depends on $z$ only. For the sake of simplicity we can take this constant $c_{1}^{\prime}$ equal to one, that is $\mathcal{W}=\Psi$. Similarly, applying the operator $\partial / \partial t+\bar{U}_{0} \partial / \partial x$ to the first and second equations (1.13) we obtain that $\mathcal{U}, \mathcal{V}$ and $\Phi$ must be proportional, and so we can take $\mathcal{U}=\mathcal{V}=\Phi$. Finally the fourth and fifth equations (1.13) imply that

$$
-\frac{\hat{u}_{x}+\hat{v}_{y}}{\hat{w}}=\frac{\mathcal{W}^{\prime}}{\mathcal{U}}=c_{2}^{\prime}, \quad \frac{\hat{\phi}}{\hat{\psi}}=\frac{\Psi}{\Phi^{\prime}}=c_{3}^{\prime},
$$

\footnotetext{
${ }^{1}$ Assuming that we are willing to pay the price of a nonlocal (mode by mode) boundary condition, for increased accuracy. The necessity of nonlocal boundary conditions appears below.

${ }^{2}$ We recall that the buoyancy frequency $N$ is assumed to be constant.
} 
where $c_{2}^{\prime}, c_{3}^{\prime}$ are constant; hence $\mathcal{W}=c_{2}^{\prime} \mathcal{U}^{\prime}$ and

$$
\mathcal{U}^{\prime \prime}+\lambda^{2} \mathcal{U}=0, \quad \mathcal{W}^{\prime \prime}+\lambda^{2} \mathcal{W}=0
$$

with $\lambda^{2}=-c_{2}^{\prime} / c_{3}^{\prime}$. By (1.14), the natural boundary conditions for $w$ and $\mathcal{W}$ are $\mathcal{W}=0$ at $z=0$ and $-L_{3}$; thus $\mathcal{U}$ and $\mathcal{W}$ are solutions of the two-point boundary value problems consisting of (1.16) and

$$
\mathcal{U}^{\prime}(0)=\mathcal{U}^{\prime}\left(-L_{3}\right)=\mathcal{W}(0)=\mathcal{W}\left(-L_{3}\right)=0
$$

We denote by $\lambda_{n}^{2}$ the corresponding eigenvalues and write (1.18)

$$
\left\{\begin{array}{l}
\lambda_{n}=\frac{n \pi}{L_{3}}, \lambda_{n}^{2}=\frac{1}{g H_{n}}, \text { i.e. } H_{n}=\frac{L_{3}^{2}}{g n^{2} \pi^{2}}, \\
\mathcal{W}_{n}=\sqrt{\frac{2}{L_{3}}} \sin \left(\lambda_{n} z\right), \mathcal{U}_{n}=\sqrt{\frac{2}{L_{3}}} \cos \left(\lambda_{n} z\right), n \geq 1, \mathcal{U}_{0}=\frac{1}{\sqrt{L_{3}}} .
\end{array}\right.
$$

As usual the functions $\mathcal{U}_{n}, \mathcal{W}_{n}$ have been chosen to form an orthonormal set in $L^{2}\left(-L_{3}, 0\right)$.

The equations satisfied by $\hat{u}, \hat{v}$, etc., will appear below. Indeed having found these special solutions to equation (1.13), we now look for the general solution in the form

$$
\left\{\begin{array}{l}
(u, v, \phi)=\sum_{n \geq 0} \mathcal{U}_{n}(z)\left(u_{n}, v_{n}, \phi_{n}\right)(x, y, t) \\
(w, \psi)=\sum_{n \geq 1} \mathcal{W}_{n}(z)\left(w_{n}, \psi_{n}\right)(x, y, t) .
\end{array}\right.
$$


Substituting these expressions in (1.13), we arrive at the following systems, for $n \geq 1$,

$$
\left\{\begin{array}{l}
\frac{\partial u_{n}}{\partial t}+\bar{U}_{0} \frac{\partial u_{n}}{\partial x}-f v_{n}+\frac{\partial \phi_{n}}{\partial x}=0 \\
\frac{\partial v_{n}}{\partial t}+\bar{U}_{0} \frac{\partial v_{n}}{\partial x}+f u_{n}+\frac{\partial \phi_{n}}{\partial y}=0 \\
\frac{\partial \psi_{n}}{\partial t}+\bar{U}_{0} \frac{\partial \psi_{n}}{\partial x}+N^{2} w_{n}=0 \\
\phi_{n}=-\frac{1}{\lambda_{n}} \psi_{n}, \quad w_{n}=-\frac{1}{\lambda_{n}}\left(\frac{\partial u_{n}}{\partial x}+\frac{\partial v_{n}}{\partial y}\right) .
\end{array}\right.
$$

For $n=0, w_{0}=\psi_{0}=0$ and there remains

$$
\left\{\begin{array}{l}
\frac{\partial u_{0}}{\partial t}+\bar{U}_{0} \frac{\partial u_{0}}{\partial x}-f v_{0}+\frac{\partial \phi_{0}}{\partial x}=0 \\
\frac{\partial v_{0}}{\partial t}+\bar{U}_{0} \frac{\partial v_{0}}{\partial x}+f u_{0}+\frac{\partial \phi_{0}}{\partial y}=0 \\
\frac{\partial u_{0}}{\partial x}+\frac{\partial v_{0}}{\partial y}=0
\end{array}\right.
$$

Note that, since the considered problem is linear, there is no coupling between the equations of modes $m$ and $n$ for $m \neq n$.

In the sequel, we will always study the barotropic mode $(n=0)$ separately and, for $n \geq 1$, we use the last two equations (1.20) and rewrite the first three equations in the form

$$
\left\{\begin{array}{l}
\frac{\partial u_{n}}{\partial t}+\bar{U}_{0} \frac{\partial u_{n}}{\partial x}-f v_{n}-\frac{1}{\lambda_{n}} \frac{\partial \psi_{n}}{\partial x}=0 \\
\frac{\partial v_{n}}{\partial t}+\bar{U}_{0} \frac{\partial v_{n}}{\partial x}+f u_{n}-\frac{1}{\lambda_{n}} \frac{\partial \psi_{n}}{\partial y}=0 \\
\frac{\partial \psi_{n}}{\partial t}+\bar{U}_{0} \frac{\partial \psi_{n}}{\partial x}-\frac{N^{2}}{\lambda_{n}}\left(\frac{\partial u_{n}}{\partial x}+\frac{\partial v_{n}}{\partial y}\right)=0
\end{array}\right.
$$

As indicated before, our aim is to propose boundary conditions for (1.20)-(1.22) which make these equations well-posed and consequently the equations (1.13) also. As we shall see in Sections 2-4 below (see also Rousseau, Temam, and Tribbia (2005b)), the boundary conditions 
are different depending on whether

$$
1 \leq n \leq n_{c}, \quad \text { or } \quad n>n_{c}
$$

where $n_{c}, \lambda_{n_{c}}$ are such that

$$
\frac{n_{c} \pi}{L_{3}}=\lambda_{n_{c}}<\frac{N}{\bar{U}_{0}}<\lambda_{n_{c}+1}=\frac{\left(n_{c}+1\right) \pi}{L_{3}} .
$$

We will not study the non generic case where $L_{3} N / \pi \bar{U}_{0}$ is an integer, that is we will assume throughout that

$$
\bar{U}_{0} \neq \frac{N}{\lambda_{n}}, \forall n \geq 1 \text {, or equivalently } \frac{N L_{3}}{\pi \bar{U}_{0}} \notin \mathbb{N} \text {. }
$$

The modes $0 \leq n \leq n_{c}$ are called subcritical, and the modes $n>$ $n_{c}$ are called supercritical. It is convenient to introduce the sub and supercritical components of the functions defined by:

$$
u^{0}=P_{0} u=\mathcal{U}_{0} u_{0}, u^{I}=P_{I} u=\sum_{n=1}^{n_{c}} \mathcal{U}_{n} u_{n}, \quad u^{I I}=P_{I I} u=\sum_{n>n_{c}}^{\infty} \mathcal{U}_{n} u_{n}
$$

and similarly for all the other functions; of course the zero mode $u^{0}$ is a subcritical mode, but, as we will see, we need to treat it separately. With these notations, the equations (1.13), (1.20), (1.22) are equivalent to the following system:

$$
\begin{gathered}
\left\{\begin{array}{c}
u_{t}^{0}+\bar{U}_{0} u_{x}^{0}-f v^{0}+\phi_{x}^{0}=0, \\
v_{t}^{0}+\bar{U}_{0} v_{x}^{0}+f u^{0}+\phi_{y}^{0}=0, \\
u_{x}^{0}+v_{y}^{0}=0,
\end{array}\right. \\
\left\{\begin{array}{c}
u_{t}^{I}+\bar{U}_{0} u_{x}^{I}-f v^{I}+\phi_{x}^{I}=0, \\
v_{t}^{I}+\bar{U}_{0} v_{x}^{I}+f u^{I}+\phi_{y}^{I}=0, \\
\psi_{t}^{I}+\bar{U}_{0} \psi_{x}^{I}+N^{2} w^{I}=0,
\end{array}\right. \\
\left\{\begin{array}{c}
u_{t}^{I I}+\bar{U}_{0} u_{x}^{I I}-f v^{I I}+\phi_{x}^{I I}=0, \\
v_{t}^{I I}+\bar{U}_{0} u_{x}^{I I}+f u^{I I}+\phi_{y}^{I I}=0, \\
\psi_{t}^{I I}+\bar{U}_{0} \psi_{x}^{I I}+N^{2} w^{I I}=0,
\end{array}\right.
\end{gathered}
$$


with the additional relations $\phi=\phi(\psi), w=w(u, v)$ :

$$
\left\{\begin{array}{l}
\phi^{I}=-\sum_{n=1}^{n_{c}} \frac{1}{\lambda_{n}} \psi_{n} \mathcal{U}_{n}, \quad w^{I}=-\sum_{n=1}^{n_{c}} \frac{1}{\lambda_{n}}\left(u_{n x}+v_{n y}\right) \mathcal{W}_{n} \\
\phi^{I I}=-\sum_{n>n_{c}} \frac{1}{\lambda_{n}} \psi_{n} \mathcal{U}_{n}, \quad w^{I I}=-\sum_{n>n_{c}} \frac{1}{\lambda_{n}}\left(u_{n x}+v_{n y}\right) \mathcal{W}_{n}
\end{array}\right.
$$

We will also set $U=(u, v, \psi), U^{0}=P_{0} U, U^{I}=P_{I} U, U^{I I}=P_{I I} U$.

1.3. Position of the Problem. Let us focus on system (1.22) for the moment. Considering for the sake of simplicity that the functions do not depend on $y$ (see Section 2 below), one can notice that the characteristic values of the resulting system are $\left(\bar{U}_{0}, \bar{U}_{0}-N / \lambda_{n}, \bar{U}_{0}+N / \lambda_{n}\right)$. Since $\bar{U}_{0}>0, N / \lambda_{n}>0$, we always have at least two positive eigenvalues. But $\bar{U}_{0}-N / \lambda_{n}$ can either be positive or negative ${ }^{3}$. We say that the corresponding mode is supercritical in the first case and subcritical in the second case (see above); it appears then that the subcritical modes require two boundary conditions on the left of the domain $(x=0)$ and one boundary condition on the right $\left(x=L_{1}\right)$, whereas the supercritical modes require three boundary conditions at $x=0$. Based on this remark, Oliger and Sundström concluded in Oliger and Sundström (1978) that the boundary value problem associated with (1.13) is ill-posed for any set of local boundary conditions (see also Temam and Tribbia (2003)).

Hereafter, our aim will be to study separately the subcritical and supercritical modes, proposing suitable boundary conditions for them, and to combine them and obtain existence, uniqueness and regularity of solutions for the whole linearized problem. In each case we will study one (subcritical/supercritical) mode separately and then combine them for the whole subcritical and supercritical components.

\section{Two Dimensionnal $x-z$ Case}

We start our study with the $2 D(x, z)$ model, for which both theoretical and numerical results have been established. We first rewrite the equations, assuming that the functions do not depend on the $y$ variable, both in their nonlinear and linear formulations. Then, a wellposedness theorem is established in Section 2.3 for the linearized PEs.

\footnotetext{
${ }^{3}$ In the ocean, taking e.g. the following values $\bar{U}_{0}=1 \mathrm{~m} . \mathrm{s}^{-1}, N=0.001 \mathrm{~s}^{-1}$ and $L_{3}=1000 \mathrm{~m}$, we end up with three subcritical modes. Realistic physical situations usually lead to a number $n_{c}$ of subcritical modes between one and five, see Temam and Tribbia (2003) for more details.
} 
After introducing the numerical scheme that is used, we end this study of the 2D-case with some numerical simulations for the linear and nonlinear cases that achieve two objectives. On the one hand the absence of blow-up in these computations indicates that the nonlinear inviscid PEs are well-posed when supplemented with the boundary conditions that we propose. On the other hand they show a very good coincidence on the subdomain $\Omega_{1}$ of the two solutions, thus showing also the computational relevance of these new boundary conditions ${ }^{4}$.

2.1. Linear and Nonlinear 2D Primitive Equations. Let us consider the nonlinear primitive equations without viscosity (1.11), without any dependance on the $y$ variable:

$$
\begin{aligned}
\frac{\partial u}{\partial t}+u \frac{\partial u}{\partial x}+w \frac{\partial u}{\partial z}-f v+\frac{\partial \phi}{\partial x} & =F_{u}, \\
\frac{\partial v}{\partial t}+u \frac{\partial v}{\partial x}+w \frac{\partial v}{\partial z}+f u & =F_{v}, \\
\frac{\partial \psi}{\partial t}+u \frac{\partial \psi}{\partial x}+\left(N^{2}+\frac{\partial \psi}{\partial z}\right) w & =F_{\psi}, \\
\frac{\partial \phi}{\partial z}=-\frac{\rho}{\rho_{0}} g & =\psi, \\
\frac{\partial u}{\partial x}+\frac{\partial w}{\partial z} & =0 .
\end{aligned}
$$

We will consider the flow in the $2 \mathrm{D}$ domain $\mathcal{M}=\left(0, L_{1}\right) \times\left(-L_{3}, 0\right)$, and supplement equation (2.1) with an initial data $u_{0}, v_{0}, \psi_{0}$. The top and bottom boundary conditions (just imposed by kinematics) are the same as for the complete 3D problem (see Section 1 above):

$$
w\left(x, z=-L_{3}, t\right)=w(x, z=0, t)=0, \quad \forall x \in\left(0, L_{1}\right), t>0 .
$$

and we also have:

$$
\begin{aligned}
\frac{\partial \phi}{\partial x}(x, z) & =\phi_{s}^{\prime}(x)-\int_{z}^{0} \frac{\partial \psi}{\partial x}\left(x, z^{\prime}\right) d z^{\prime}, \\
w(x, z) & =\int_{z}^{0} \frac{\partial u}{\partial x}\left(x, z^{\prime}\right) d z^{\prime}
\end{aligned}
$$

where $\phi_{s}(x, t)=\phi(x, z=0, t)$ is the surface pressure divided by $\rho_{0}$, and $\phi_{s}^{\prime}$ its derivative with respect to $x$.

\footnotetext{
${ }^{4}$ See Section 2.4 for the full description of this numerical test.
} 
The 2D PEs (2.1), linearized around the stratified flow $\overline{\mathbf{v}}_{0}=\bar{U}_{0} e_{x}, \bar{\rho}, \bar{T}, \bar{p}$, read:

$$
\begin{aligned}
\frac{\partial u}{\partial t}+\bar{U}_{0} \frac{\partial u}{\partial x}-f v+\frac{\partial \phi}{\partial x} & =F_{u}, \\
\frac{\partial v}{\partial t}+\bar{U}_{0} \frac{\partial v}{\partial x}+f u & =F_{v}-f \bar{U}_{0}, \\
\frac{\partial \psi}{\partial t}+\bar{U}_{0} \frac{\partial \psi}{\partial x}+N^{2} w & =F_{\psi}, \\
\frac{\partial \phi}{\partial z}=-\frac{\rho}{\rho_{0}} g & =\psi, \\
\frac{\partial u}{\partial x}+\frac{\partial w}{\partial z} & =0 .
\end{aligned}
$$

The aim of this section is to consider some lateral boundary conditions at $x=0$ and $x=L_{1}$ that are both physically reasonable and computationnally satisfying, and that lead to the well-posedness of the problem (2.5).

\subsection{The Modal Equations and Boundary Conditions.}

We consider a normal mode decomposition of the solution of the following form (see Section 1 above for the details and the justifications):

$$
\begin{aligned}
(u, v, \phi) & =\sum_{n \geq 0} \mathcal{U}_{n}(z)\left(u_{n}, v_{n}, \phi_{n}\right)(x, t), \\
(w, \psi) & =\sum_{n \geq 1} \mathcal{W}_{n}(z)\left(w_{n}, \psi_{n}\right)(x, t) .
\end{aligned}
$$

We now introduce the expansion (2.6)-(2.7) into equations (2.5). We multiply $(2.5 \mathrm{a}),(2.5 \mathrm{~b})$ and $(2.5 \mathrm{e})$ by $\mathcal{U}_{n},(2.5 \mathrm{c})$ and $(2.5 \mathrm{~d})$ by $\mathcal{W}_{n}$ and integrate on $\left(-L_{3}, 0\right)$, and we find the same equations (1.20) but with no depence on the $y$ variable, namely, for $n \geq 1$ :

$$
\left\{\begin{aligned}
\frac{\partial u_{n}}{\partial t}+\bar{U}_{0} \frac{\partial u_{n}}{\partial x}-f v_{n}+\frac{\partial \phi_{n}}{\partial x} & =F_{u, n} \\
\frac{\partial v_{n}}{\partial t}+\bar{U}_{0} \frac{\partial v_{n}}{\partial x}+f u_{n} & =F_{v, n} \\
\frac{\partial \psi_{n}}{\partial t}+\bar{U}_{0} \frac{\partial \psi_{n}}{\partial x}+N^{2} w_{n} & =F_{\psi, n} \\
\phi_{n} & =-\frac{1}{\lambda_{n}} \psi_{n} \\
w_{n} & =-\frac{1}{\lambda_{n}} \frac{\partial u_{n}}{\partial x}
\end{aligned}\right.
$$


The constant mode in $z(\mathrm{n}=0)$ is different (simpler), and we postpone its study to Section 2.3.5 below. Taking into account the last two equations of (2.8) the first three become:

$$
\left\{\begin{aligned}
\frac{\partial u_{n}}{\partial t}+\bar{U}_{0} \frac{\partial u_{n}}{\partial x}-f v_{n}-\frac{1}{\lambda_{n}} \frac{\partial \psi_{n}}{\partial x} & =F_{u, n} \\
\frac{\partial v_{n}}{\partial t}+\bar{U}_{0} \frac{\partial v_{n}}{\partial x}+f u_{n} & =F_{v, n} \\
\frac{\partial \psi_{n}}{\partial t}+\bar{U}_{0} \frac{\partial \psi_{n}}{\partial x}-\frac{N^{2}}{\lambda_{n}} \frac{\partial u_{n}}{\partial x} & =F_{\psi, n}
\end{aligned}\right.
$$

For the nonlinear PEs, the normal modes decomposition reads

$$
\begin{array}{r}
\frac{\partial u_{n}}{\partial t}+\bar{U}_{0} \frac{\partial u_{n}}{\partial x}-f v_{n}+\frac{\partial \phi_{n}}{\partial x}+B_{u, n}(U)=F_{u, n} \\
\frac{\partial v_{n}}{\partial t}+\bar{U}_{0} \frac{\partial v_{n}}{\partial x}+f u_{n}+B_{v, n}(U)=F_{v, n} \\
\frac{\partial \psi_{n}}{\partial t}+\bar{U}_{0} \frac{\partial \psi_{n}}{\partial x}+N^{2} w_{n}+B_{\psi, n}(U)=F_{\psi, n}
\end{array}
$$

where $B_{u, n}, B_{v, n}$ and $B_{\psi, n}$ are the following modal parts of the nonlinearities:

$$
\begin{aligned}
B_{u, n} & =\int_{-L_{3}}^{0}\left(u \frac{\partial u}{\partial x}+w \frac{\partial u}{\partial z}\right) \mathcal{U}_{n} d z \\
B_{v, n} & =\int_{-L_{3}}^{0}\left(u \frac{\partial v}{\partial x}+w \frac{\partial v}{\partial z}\right) \mathcal{U}_{n} d z, \\
B_{\psi, n} & =\int_{-L_{3}}^{0}\left(u \frac{\partial \psi}{\partial x}+w \frac{\partial \psi}{\partial z}\right) \mathcal{W}_{n} d z
\end{aligned}
$$

with $u, v, \psi, w$ truncated to $M$ modes.

Let us now introduce the lateral boundary conditions which, for each $n \geq 1$, will supplement this system. We recall (see Section 1.3 above) that lateral boundary conditions at $x=0$ and $x=L_{1}$ cannot be imposed without separating the subcritical and supercritical modes.

The boundary conditions for the subcritical modes were discussed in Rousseau, Temam, and Tribbia (2005a, 2004), they are recalled below. The boundary conditions for the supercritical modes are less problematic, we now present them. For $n>n_{c}$, a set of natural boundary conditions for system (2.9) is:

$$
\left\{\begin{array}{c}
u_{n}(0, t)=0, \\
v_{n}(0, t)=0, \\
\psi_{n}(0, t)=0 .
\end{array}\right.
$$


In (2.12) and (2.14) we chose, for simplicity, homogeneous boundary conditions, but we discuss in Section 2.3.4 below the case of nonzero boundary values.

For $1 \leq n \leq n_{c}, \bar{U}_{0}-N / \lambda_{n}<0$, and the corresponding eigenvector is $\eta_{n}=u_{n}+\psi_{n} / N$. The eigenvectors related to $\bar{U}_{0}$ and $\bar{U}_{0}+N / \lambda_{n}$ are respectively $v_{n}$ and $\xi_{n}=u_{n}-\psi_{n} / N$. Thanks to (1.20), we have, for $n \geq 1,\left(\xi_{n}, \eta_{n}\right)=\left(u_{n}+\lambda_{n} \phi_{n}, u_{n}-\lambda_{n} \phi_{n}\right)$.

Using the variables $\xi_{n}, v_{n}, \eta_{n}$ we rewrite (2.9) as follows:

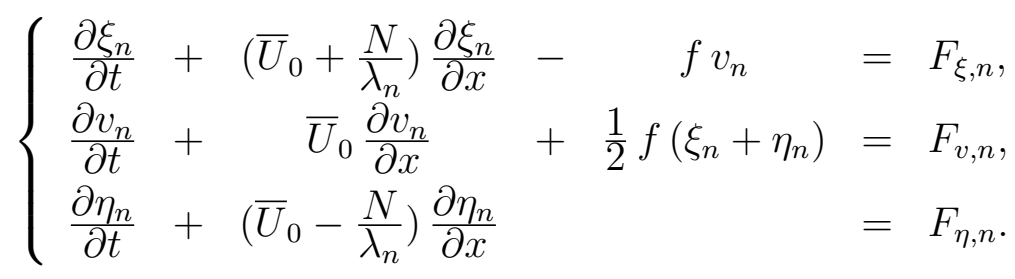

Hence, for these subcritical modes $\left(n \leq n_{c}\right)$, a set of natural and nonreflective boundary conditions is the following

$$
\left\{\begin{aligned}
\xi_{n}(0, t) & =0 \\
v_{n}(0, t) & =0 \\
\eta_{n}\left(L_{1}, t\right) & =0
\end{aligned}\right.
$$

In Section 2.3, we will prove the well-posedness of the linear Primitive Equations (2.5) (equivalent mode by mode to (2.8)) with the modal boundary conditions (2.12) and (2.14).

For the nonlinear case, we perform the same change of variables $\xi_{n}=u_{n}-\psi_{n} / N, \eta_{n}=u_{n}+\psi_{n} / N$, and obtain the nonlinear version of (2.13), namely:

$$
\begin{gathered}
\frac{\partial \xi_{n}}{\partial t}+\left(\bar{U}_{0}+\frac{N}{\lambda_{n}}\right) \frac{\partial \xi_{n}}{\partial x}-f v_{n}+B_{\xi, n}(U)=F_{\xi, n} \\
\frac{\partial v_{n}}{\partial t}+\bar{U}_{0} \frac{\partial v_{n}}{\partial x}+f \frac{\xi_{n}+\eta_{n}}{2}+B_{v, n}(U)=F_{v, n} \\
\frac{\partial \eta_{n}}{\partial t}+\left(\bar{U}_{0}-\frac{N}{\lambda_{n}}\right) \frac{\partial \eta_{n}}{\partial x}-f v_{n}+B_{\eta, n}(U)=F_{\eta, n}
\end{gathered}
$$

where $B_{\xi, n}=B_{u, n}-B_{\psi, n} / N$ and $B_{\eta, n}=B_{u, n}+B_{\psi, n} / N$.

We assume in the following that the initial data is such that the nonlinear part is small compared to the stratified flow $\left(\bar{U}_{0}, 0,0\right)$, so that the characteristic values do not change sign, at least during a certain period of time. Assuming so, we conjecture that the boundary conditions 
provided for the linearized system will give a well-posed problem for the nonlinear equations, at least for some time. We leave the theoretical analysis to subsequent studies, and perform hereafter the corresponding numerical simulations based on this hypothesis, which is conforted by the lack of numerical blow-up. Hence the boundary conditions that we consider for the nonlinear case are also (2.12)-(2.14).

2.3. Well-Posedness Results. We aim to implement the boundary conditions (2.12) and (2.14) in the linear case, and we first set the functional framework appropriate for these boundary conditions.

2.3.1. Theoretical Framework. We aim to write the initial value problem under consideration as a functional evolution in an appropriate Hilbert space $H$ :

$$
\left\{\begin{array}{l}
\frac{d U}{d t}+A U=F, \\
U(0)=U_{0} .
\end{array}\right.
$$

Here $A$ is an unbounded operator with domain $D(A) \subset H$, the forcing $F$ taking is values in $H$ and the initial data $U_{0} \in D(A)$ are given.

We define $H$ by setting

$$
\begin{gathered}
H=H_{u} \times H_{v} \times H_{\psi}, \\
H_{u}=\left\{u \in L^{2}(\mathcal{M}) / \int_{-L_{3}}^{0} u(x, z) d z=0 \text { a.e. on }\left(0, L_{1}\right)\right\}, \\
H_{v}=H_{\psi}=L^{2}(\mathcal{M}),
\end{gathered}
$$

where $\mathcal{M}$ is the $2 \mathrm{D}$ domain $\left(0, L_{1}\right) \times\left(-L_{3}, 0\right)$. We endow $H$ with the scalar product ${ }^{5}$

$$
(U, \widetilde{U})_{H}=\int_{\mathcal{M}}\left(u \widetilde{u}+v \widetilde{v}+\frac{1}{N^{2}} \psi \widetilde{\psi}\right) d \mathcal{M}, \quad \forall(U, \widetilde{U}) \in H^{2},
$$

and the associated norm

$$
|U|_{H}=\left\{(U, U)_{H}\right\}^{1 / 2}, \quad \forall U \in H .
$$

The space $H_{u}$ is clearly closed in $L^{2}(\mathcal{M})$, and $H=H_{u} \times H_{v} \times H_{\psi}$ is a closed subspace of $\left(L^{2}(\mathcal{M})\right)^{3}$, which we endow with the scalar product and norm derived from $(2.18)$ and equivalent to those of $\left(L^{2}(\mathcal{M})\right)^{3}$. We

\footnotetext{
${ }^{5}$ It is not surprising to have $1 / N^{2}$ as a multiplicative coefficient in front of the last term of $(U, \widetilde{U})_{H}$, since $\int_{\mathcal{M}}\left(u^{2}+v^{2}\right) d \mathcal{M}$ represents the kinetic energy whereas $N^{-2} \int_{\mathcal{M}} \psi^{2} d \mathcal{M}$ is the available potential energy so that the square of the norm in $H$ represents the total energy.
} 
denote by $P$ the orthogonal projector from $L^{2}(\mathcal{M})$ onto $H_{u}$. For every $g \in L^{2}(\mathcal{M})$

$$
\begin{array}{r}
P(g)(x, z)=g(x, z)-\frac{1}{L_{3}} \int_{-L_{3}}^{0} g\left(x, z^{\prime}\right) d z^{\prime} \\
(I-P)(g)(x, z)=\frac{1}{L_{3}} \int_{-L_{3}}^{0} g\left(x, z^{\prime}\right) d z^{\prime}
\end{array}
$$

It is easily checked that $P g \in H_{u}$ and $(I-P) g \perp P g$. Finally $H_{u}^{\perp}$ is identical to $L_{x}^{2}\left(0, L_{1}\right)$. Indeed for $g \in H_{u}^{\perp},(I-P) g=g$, so that $g$ does not depend on $z$ and belongs to $L_{x}^{2}\left(0, L_{1}\right)$. Conversely if $h \in L_{x}^{2}\left(0, L_{1}\right)$, then for every $u \in H_{u},(u, h)_{L^{2}(\mathcal{M})}=\int_{0}^{L_{1}} h(x) \int_{-L_{3}}^{0} u(x, z) d z d x=0$ and $h \in H_{u}^{\perp}$.

We are now in position to define the operator $A$; its domain $D(A)$ is defined by

$$
D(A)=\left\{\begin{array}{l|l}
U=(u, v, \psi) \in H / & \begin{array}{l}
\left(u_{x}, v_{x}, \psi_{x}\right) \in L^{2}(\mathcal{M}) \\
(u, v, \psi) \text { verify }(2.23) \text { and }(2.24)
\end{array}
\end{array}\right\} .
$$

Here and in the sequel $u_{x}, u_{z}$ denote the partial derivatives $\partial u / \partial x$, $\partial u / \partial z$ of a function $u$.

The boundary conditions (2.23) and (2.24), identical to (2.12) and $(2.14)$, are written in the following form ${ }^{6}$. For the subcritical modes $\left(1 \leq n \leq n_{c}\right)$ :

$$
\left\{\begin{array}{l}
\int_{-L_{3}}^{0} u(0, z) \mathcal{U}_{n}(z) d z-\frac{1}{N} \int_{-L_{3}}^{0} \psi(0, z) \mathcal{W}_{n}(z) d z=0 \\
\int_{-L_{3}}^{0} v(0, z) \mathcal{U}_{n}(z) d z=0 \\
\int_{-L_{3}}^{0} u\left(L_{1}, z\right) \mathcal{U}_{n}(z) d z+\frac{1}{N} \int_{-L_{3}}^{0} \psi\left(L_{1}, z\right) \mathcal{W}_{n}(z) d z=0
\end{array}\right.
$$

\footnotetext{
${ }^{6}$ We note that the boundary conditions on $v$ do not depend on the modes (see also the boundary condition on the constant mode $v_{0}$ in Section 2.3.5 below), hence they could be written in the form $v(0, z)=0, \forall z \in\left(-L_{3}, 0\right)$. However we keep the modal notation by analogy with the other functions $u$ and $\psi$, and because this is the way this boundary condition is actually implemented in numerical simulations, see Section 2.5 below
} 
and for the supercritical ones $\left(n>n_{c}\right)$ :

$$
\left\{\begin{array}{l}
\int_{-L_{3}}^{0} u(0, z) \mathcal{U}_{n}(z) d z=0 \\
\int_{-L_{3}}^{0} v(0, z) \mathcal{U}_{n}(z) d z=0 \\
\int_{-L_{3}}^{0} \psi(0, z) \mathcal{W}_{n}(z) d z=0
\end{array}\right.
$$

For every $U=(u, v, \psi) \in D(A), A U$ is given by:

$$
A U=\left(\begin{array}{l}
P\left[\bar{U}_{0} u_{x}-f v-\int_{z}^{0} \psi_{x}\left(x, z^{\prime}\right) d z^{\prime}\right] \\
\bar{U}_{0} v_{x}+f u \\
\bar{U}_{0} \psi_{x}+N^{2} w
\end{array}\right)
$$

where $w=w(u)$ is given by $(2.4)$.

We now intend to prove the well-posedness of equation (2.16), corresponding to the linearized PEs supplemented with the boundary conditions (2.23) and (2.24), in the context of the linear semi-group theory.

2.3.2. Main Theorem. To prove the well-posedness of the initial value problem (2.16), we will use the following version of the Hille-Yosida theorem borrowed from Burq and Gérard (2003) (see also Brézis (1973), Henry (1981),Lions (1965),Pazy (1983), Yosida (1980)):

Theorem 2.1. (Hille-Yosida Theorem) Let $H$ be a Hilbert space and let $A: D(A) \longrightarrow H$ be a linear unbounded operator, with domain $D(A) \subset H$. Assume the following :

(i) $D(A)$ is dense in $H$ and $A$ is closed,

(ii) $A$ is $\geq 0$, i.e. $(A U, U)_{H} \geq 0, \quad \forall U \in D(A)$,

(iii) $\exists \mu_{0}>0$, such that $A+\mu_{0} I$ is onto.

Then $-A$ is infinitesimal generator of a semigroup of contractions $\{S(t)\}_{t \geq 0}$ in $H$, and for every $U_{0} \in H$ and $F \in L^{1}(0, T ; H)$, there exists a unique solution $U \in \mathcal{C}([0, T] ; H)$ of $(2.16)$,

$$
U(t)=S(t) U_{0}+\int_{0}^{t} S(t-s) F(s) d s .
$$

If furthermore $U_{0} \in D(A)$ and $F^{\prime}=d F / d t \in L^{1}(0, T ; H)$ then $U$ satisfies (2.16) and

$$
U \in \mathcal{C}([0, T] ; H) \cap L^{\infty}(0, T ; D(A)), \quad \frac{d U}{d t} \in L^{\infty}(0, T ; H) .
$$


We now state and prove the main result for the homogeneous boundary conditions in 2D:

Theorem 2.2. Let $H$ be the Hilbert space defined in (2.17) and $A$ be the linear operator defined in (2.25) corresponding to the linearized Primitive Equations with vanishing viscosity and homogeneous modal boundary conditions.

Then the initial value problem (2.16), corresponding to equations (2.5) supplemented with the boundary conditions (2.23) and (2.24) is

well-posed, that is for every initial data $U_{0} \in D(A)$ and forcing $F \in$ $L^{1}(0, T ; H)$, there exists a unique solution $U \in \mathcal{C}([0, T] ; H)$ of $(2.16)$.

\subsubsection{Proof of Theorem 2.2.}

We want to apply Theorem 2.1 to equation (2.16). To this aim we verify the hypotheses $(i),(i i)$ and $($ iii $)$ of the Hille-Yosida theorem (Theorem 2.1); we start with (ii) and (iii), and postpone the proof of (i) to Lemma 2.3 below. We start with the proof of $(i i)$ :

Lemma 2.1. For every $U \in D(A),(A U, U)_{H} \geq 0$.

Proof. For any $U \in H$, let us compute the scalar product $(A U, U)_{H}$ :

$$
\begin{aligned}
(A U, U)_{H}= & \int_{\mathcal{M}} P\left(\bar{U}_{0} u_{x}-f v-\int_{z}^{0} \psi_{x}\left(x, z^{\prime}\right) d z^{\prime}\right) u d \mathcal{M} \\
& +\int_{\mathcal{M}}\left(\bar{U}_{0} v_{x}+f u\right) v d \mathcal{M}+\int_{\mathcal{M}}\left(\bar{U}_{0} \psi_{x}+N^{2} w\right) \frac{\psi}{N^{2}} d \mathcal{M} .
\end{aligned}
$$


Since $u \in H_{u}$, we have, using (2.4):

$$
\begin{aligned}
(A U, U)_{H}= & \int_{\mathcal{M}}\left(\bar{U}_{0} u_{x}-f v-\int_{z}^{0} \psi_{x}\left(x, z^{\prime}\right) d z^{\prime}\right) u d \mathcal{M} \\
& +\int_{\mathcal{M}}\left(\bar{U}_{0} v_{x}+f u\right) v d \mathcal{M} \\
& +\int_{\mathcal{M}}\left(\bar{U}_{0} \psi_{x}+N^{2} w\right) \frac{\psi}{N^{2}} d \mathcal{M} \\
= & \int_{-L_{3}}^{0} \frac{\bar{U}_{0}}{2}\left(u^{2}\left(L_{1}\right)-u^{2}(0)+v^{2}\left(L_{1}\right)-v^{2}(0)\right. \\
& \left.+\frac{1}{N^{2}} \psi^{2}\left(L_{1}\right)-\frac{1}{N^{2}} \psi^{2}(0)\right) d z \\
& -\int_{\mathcal{M}}\left\{u(x, z) \int_{z}^{0} \psi_{x}\left(x, z^{\prime}\right) d z^{\prime}\right. \\
& \left.-\psi(x, z) \int_{z}^{0} u_{x}\left(x, z^{\prime}\right) d z^{\prime}\right\} d x d z .
\end{aligned}
$$

Here $u\left(L_{1}\right), u(0)$ stand for $u\left(L_{1}, z\right), u(0, z)$, etc. Using the expansion (2.6), (2.7) with (1.18), it is easy to check that:

$$
\left\{\begin{aligned}
-\int_{z}^{0} \psi_{x}\left(x, z^{\prime}\right) d z^{\prime} & =\sum_{n \geq 1} \frac{\psi_{n x}(x)}{\lambda_{n}}\left(1-\mathcal{U}_{n}(z)\right) \\
& =\theta(x)-\sum_{n \geq 1} \frac{\psi_{n x}(x)}{\lambda_{n}} \mathcal{U}_{n}(z) \\
\int_{z}^{0} u_{x}\left(x, z^{\prime}\right) d z^{\prime} & =-\sum_{n \geq 1} \frac{u_{n x}(x)}{\lambda_{n}} \mathcal{W}_{n}(z)
\end{aligned}\right.
$$

where $\theta=\theta(x)$ is an $L^{2}$-function depending only on $x$. 
Using again the expansion (2.6), (2.7), and remembering that $u \in$ $H_{u}$, the integral $\int_{\mathcal{M}} u \theta d \mathcal{M}$ vanishes and we find:

$$
\begin{aligned}
(A U, U)_{H}= & \sum_{n \geq 1} \frac{\bar{U}_{0}}{2}\left(u_{n}^{2}\left(L_{1}\right)-u_{n}^{2}(0)+v_{n}^{2}\left(L_{1}\right)-v_{n}^{2}(0)\right. \\
& \left.+\frac{1}{N^{2}} \psi_{n}^{2}\left(L_{1}\right)-\frac{1}{N^{2}} \psi_{n}^{2}(0)\right) \\
& +\frac{\bar{U}_{0}}{2}\left(v_{0}^{2}\left(L_{1}\right)-v_{0}^{2}(0)\right) \\
& -\sum_{n \geq 1} \frac{1}{\lambda_{n}} \int_{0}^{L_{1}}\left(\psi_{n x} u_{n}+\psi_{n} u_{n x}\right) d x
\end{aligned}
$$

Using the boundary conditions (2.23) for the subcritical modes and (2.24) for the supercritical ones, we find:

$$
\begin{aligned}
(A U, U)_{H}= & \sum_{1 \leq n \leq n_{c}} \frac{\bar{U}_{0}}{2}\left(u_{n}^{2}\left(L_{1}\right)-u_{n}^{2}(0)+v_{n}^{2}\left(L_{1}\right)+u_{n}^{2}\left(L_{1}\right)-u_{n}^{2}(0)\right) \\
& +\frac{\bar{U}_{0}}{2} v_{0}^{2}\left(L_{1}\right)+\sum_{1 \leq n \leq n_{c}} \frac{N}{\lambda_{n}}\left(u_{n}^{2}\left(L_{1}\right)+u_{n}^{2}(0)\right) \\
& +\sum_{n>n_{c}} \frac{\bar{U}_{0}}{2}\left(u_{n}^{2}\left(L_{1}\right)+v_{n}^{2}\left(L_{1}\right)+\frac{1}{N^{2}} \psi_{n}^{2}\left(L_{1}\right)\right) \\
& -\sum_{n>n_{c}} \frac{1}{\lambda_{n}} u_{n}\left(L_{1}\right) \psi_{n}\left(L_{1}\right) .
\end{aligned}
$$

For every subcritical mode (when $n \leq n_{c}$ ):

$$
\begin{aligned}
& \bar{U}_{0}\left(u_{n}^{2}\left(L_{1}\right)-u_{n}^{2}(0)+\frac{1}{2} v_{n}^{2}\left(L_{1}\right)\right)+\frac{N}{\lambda_{n}}\left(u_{n}^{2}\left(L_{1}\right)+u_{n}^{2}(0)\right) \\
= & \left(\bar{U}_{0}+\frac{N}{\lambda_{n}}\right) u_{n}^{2}\left(L_{1}\right)+\frac{\bar{U}_{0}}{2} v_{n}^{2}\left(L_{1}\right)+\left(\frac{N}{\lambda_{n}}-\bar{U}_{0}\right) u_{n}^{2}(0) \geq 0 ;
\end{aligned}
$$

the latter quantity is nonnegative, thanks to the definition of $n_{c}$. For every supercritical mode (when $n>n_{c}$ ):

$$
\begin{aligned}
& \frac{\bar{U}_{0}}{2}\left(u_{n}^{2}\left(L_{1}\right)+v_{n}^{2}\left(L_{1}\right)+\frac{1}{N^{2}} \psi_{n}^{2}\left(L_{1}\right)\right)-\frac{1}{\lambda_{n}} u_{n}\left(L_{1}\right) \psi_{n}\left(L_{1}\right) \\
= & \frac{\bar{U}_{0}}{2} v_{n}^{2}\left(L_{1}\right)+\frac{\bar{U}_{0}}{2}\left(u_{n}\left(L_{1}\right)-\frac{N}{\bar{U}_{0} \lambda_{n}} \psi_{n}\left(L_{1}\right)\right)^{2} \\
& +\frac{\bar{U}_{0}}{2 N^{2}}\left(1-\frac{N^{2}}{\bar{U}_{0}^{2} \lambda_{n}^{2}}\right) \psi_{n}^{2}\left(L_{1}\right) \geq 0 .
\end{aligned}
$$


This quantity is also nonnegative, which achieves the proof of Lemma 2.1 .

Due to (1.24), which is assumed throughout, we can choose $\mu_{0}$ such that:

$$
\begin{array}{r}
\mu_{0} \neq f^{2}\left(1-\bar{U}_{0}^{2} \lambda_{n}^{2} / N^{2}\right), \quad \forall n \geq 1 \\
\mu_{0} \neq f^{2} \bar{U}_{0}^{2} \lambda_{n}^{2} / N, \quad \forall n \geq 1 .
\end{array}
$$

With this choice of $\mu_{0}$, we can prove the following lemma:

Lemma 2.2. The operator $A+\mu_{0} I$ is onto from $D(A)$ onto $H$, where $\mu_{o}$ satisfies (2.29) and (2.30).

Proof. For $\mu_{0}$ as indicated, we are given $F=\left(F_{u}, F_{v}, F_{\psi}\right)$ in $H$, and we look for $U=(u, v, \psi)$ in $D(A)$ such that $\left(A+\mu_{0} I\right) U=F$. Writing this equation componentwise, we find:

$$
\left\{\begin{aligned}
\bar{U}_{0} u_{x}(x, z)-f v(x, z)+\mu_{0} u(x, z) & \\
-\int_{z}^{0} \psi_{x}\left(x, z^{\prime}\right) d z^{\prime}+\phi_{s}^{\prime}(x) & =F_{u}(x, z), \\
\bar{U}_{0} v_{x}(x, z)+f u(x, z)+\mu_{0} v(x, z) & =F_{v}(x, z), \\
\bar{U}_{0} \psi_{x}(x, z)+N^{2} w(x, z)+\mu_{0} \psi(x, z) & =F_{\psi}(x, z) .
\end{aligned}\right.
$$

To obtain the modal equations corresponding to (2.31), we multiply the three equations by $\mathcal{U}_{n}, \mathcal{U}_{n}$ and $\mathcal{W}_{n}$ respectively, and integrate on $\left(-L_{3}, 0\right)$.

Of course, since $F=\left(F_{u}, F_{v}, F_{\psi}\right) \in H$, we also have the following modal decompositions:

$$
\left\{\begin{aligned}
F_{u}(x, z) & =\sum_{n \geq 1} \mathcal{U}_{n}(z) F_{u, n}(x) \\
F_{v}(x, z) & =\sum_{n \geq 0} \mathcal{U}_{n}(z) F_{v, n}(x) \\
F_{\psi}(x, z) & =\sum_{n \geq 1} \mathcal{W}_{n}(z) F_{\psi, n}(x)
\end{aligned}\right.
$$

Note that for $F$ as for $U$, since $F_{u} \in H_{u} \subset L^{2}(\mathcal{M}), F_{u, 0}=0$ and the decomposition of $F_{u}$ starts from $n=1$.

For the barotropic mode $n=0$ (constant in the variable $z$ ), we only consider the first two equations, since multiplying the third one by $\mathcal{W}_{0}=0$ would be useless. Integrating the equation for $v$ and reporting in the equation for $u$ (in which $u_{0}=0$, see above), we find $v_{0}$ (formerly 
denoted $v_{0}$ ) and the surface pressure $\phi_{s}$, up to an additive constant $\phi_{s}(0)$ :

$$
\left\{\begin{array}{l}
v_{0}(x)=\frac{1}{\bar{U}_{0}} \int_{0}^{x} F_{v 0}\left(x^{\prime}\right) e^{\left(x^{\prime}-x\right) \mu_{0} / \bar{U}_{0}} d x^{\prime} \\
\phi_{s}(x)=\phi_{s}(0)+\int_{0}^{x}\left(f v_{0}\left(x^{\prime}\right)-\frac{L_{3}^{2}}{\pi} \sum_{n \geq 1} \psi_{n x}\left(x^{\prime}\right)\right) d x^{\prime}
\end{array}\right.
$$

Naturally, the above expression of $\phi_{s}$ depends on the other modes $(n \geq 1)$. We now write the equations, derived from (2.31) mode by mode:

$$
\left\{\begin{aligned}
\bar{U}_{0} u_{n x}-f v_{n}+\mu_{0} u_{n}-\frac{1}{\lambda_{n}} \psi_{n x} & =F_{u, n}, \\
\bar{U}_{0} v_{n x}+f u_{n}+\mu_{0} v_{n} & =F_{v, n}, \\
\bar{U}_{0} \psi_{n x}-\frac{N^{2}}{\lambda_{n}} u_{n x}+\mu_{0} \psi_{n} & =F_{\psi, n} .
\end{aligned}\right.
$$

We recall that the functions $\left(u_{n}, v_{n}, \psi_{n}\right)$ only depend on the $x$ variable. Hence (2.34) is just a linear system of ordinary differential equations for $u_{n}, v_{n}, \psi_{n}$.

As usual, to solve (2.34), we first consider the corresponding homogeneous system. Dropping the subscripts $n$ for the moment, we write:

$$
\left\{\begin{aligned}
\bar{U}_{0} \frac{\partial^{2} u}{\partial x^{2}}-f v-\frac{1}{\lambda} \frac{\partial^{2} \psi}{\partial x^{2}}+\mu_{0} u & =0 \\
\bar{U}_{0} \frac{\partial^{2} v}{\partial x^{2}}+f u & +\mu_{0} v=0 \\
\bar{U}_{0} \frac{\partial^{2} \psi}{\partial x^{2}} & -\frac{N^{2}}{\lambda} \frac{\partial^{2} u}{\partial x^{2}}+\mu_{0} \psi=0
\end{aligned}\right.
$$

The general solution of this linear system is of the form

$$
(u, v, \psi)=\sum_{i=1}^{3}\left(A_{i}, B_{i}, C_{i}\right) e^{R_{i} x}
$$

where the coefficients $R_{i}$ are as follows:

$$
\left\{\begin{array}{l}
R_{1}=-\frac{\mu_{0}}{\bar{U}_{0}}, \\
R_{2}=\frac{-\mu_{0} \bar{U}_{0}+\frac{N}{\lambda}\left(\mu_{0}^{2}-f^{2}\left(\bar{U}_{0}^{2} \lambda^{2} / N^{2}-1\right)\right)^{1 / 2}}{\bar{U}_{0}^{2}-\frac{N^{2}}{\lambda^{2}}}, \\
R_{3}=\frac{-\mu_{0} \bar{U}_{0}-\frac{N}{\lambda}\left(\mu_{0}^{2}-f^{2}\left(\bar{U}_{0}^{2} \lambda^{2} / N^{2}-1\right)\right)^{1 / 2}}{\bar{U}_{0}^{2}-\frac{N^{2}}{\lambda^{2}}} .
\end{array}\right.
$$


The coefficients $\left(A_{i}, B_{i}, C_{i}\right)_{1 \leq i \leq 3}$ satisfy the equations:

$$
\left\{\begin{array}{l}
A_{i}=a_{i} B_{i}, \\
C_{i}=c_{i} B_{i},
\end{array}\right.
$$

with

$$
\left\{\begin{array}{l}
a_{1}=0, \\
c_{1}=-\frac{f \lambda}{R_{1}},
\end{array}\right.
$$

and, for $i=2,3$ :

$$
\left\{\begin{aligned}
a_{i} & =-\frac{\bar{U}_{0} R_{i}+\mu_{0}}{f}, \\
c_{i} & =\frac{N^{2} R_{i}}{\lambda\left(\bar{U}_{0} R_{i}+\mu_{0}\right)} .
\end{aligned}\right.
$$

Now, returning to the nonhomogeneous system (2.34), we look for a solution $\left(u_{n}, v_{n}, \psi_{n}\right)=(u, v, \psi)$ of the form:

$$
Y=(u, v, \psi)^{T}=\sum_{i=1}^{3}\left(a_{i}, 1, c_{i},\right)^{T} B_{i}(x) e^{R_{i} x},
$$

where the $\left(a_{i}, c_{i}\right)$ and $R_{i}$ have been defined above. Equation (2.34) reads then:

$$
M Y^{\prime}+N Y=F
$$

where

$$
\begin{gathered}
M=\left(\begin{array}{ccc}
\bar{U}_{0} & 0 & -\frac{1}{\lambda} \\
0 & \bar{U}_{0} & 0 \\
-\frac{N^{2}}{\lambda} & 0 & \bar{U}_{0}
\end{array}\right) \quad, \quad N=\left(\begin{array}{ccc}
\mu_{0} & -f & 0 \\
f & \mu_{0} & 0 \\
0 & 0 & \mu_{0}
\end{array}\right), \\
F=\left(F_{u}, F_{v}, F_{\psi}\right)^{T} .
\end{gathered}
$$

Thanks to assumption (1.24), $\bar{U}_{0} \neq N / \lambda_{n}$, the matrix $M$ is regular and it can be inverted. Equation (2.42) then implies:

$$
\sum_{i=1}^{3}\left(a_{i}, 1, c_{i}\right)^{T} B_{i}^{\prime}(x) e^{R_{i} x}=M^{-1} F=: \widetilde{F} .
$$

We now write the latter equation component by component. We find:

$$
\Lambda(x) .\left(B_{1}^{\prime}(x), B_{2}^{\prime}(x), B_{3}^{\prime}(x)\right)^{T}=\left(\widetilde{F}_{1}(x), \widetilde{F}_{2}(x), \widetilde{F}_{3}(x)\right)^{T}
$$


with

$$
\Lambda(x)=\left(\begin{array}{ccc}
0 & a_{2} e^{R_{2} x} & a_{3} e^{R_{3} x} \\
e^{R_{1} x} & e^{R_{2} x} & e^{R_{3} x} \\
c_{1} e^{R_{1} x} & c_{2} e^{R_{2} x} & c_{3} e^{R_{3} x}
\end{array}\right)
$$

Let us check that the matrix $\Lambda(x)$ is regular for every $x \in \mathbb{R}$; it is clearly sufficient to do so for $x=0$, for which

$$
\Lambda(0)=\left(\begin{array}{ccc}
0 & a_{2} & a_{3} \\
1 & 1 & 1 \\
c_{1} & c_{2} & c_{3}
\end{array}\right)
$$

We call $\mathbf{L}_{1}, \mathbf{L}_{2}$ and $\mathbf{L}_{3}$ the lines of $\Lambda(0)$. It is clear that $\mathbf{L}_{1}$ and $\mathbf{L}_{2}$ are linearly independent vectors. Then if $\Lambda(0)$ were not regular there would exist $(\alpha, \beta) \in \mathbb{R}^{2}$ such that $\mathbf{L}_{3}=\alpha \mathbf{L}_{1}+\beta \mathbf{L}_{2}$. After some easy computations we would find that necessarily:

$$
a_{3}\left(c_{2}-c_{1}\right)=a_{2}\left(c_{3}-c_{1}\right)
$$

which leads (see (2.39) and (2.40)) to:

$$
\bar{U}_{0}\left(R_{3}-R_{2}\right) f^{2} \lambda^{2} / N^{2}=-\mu_{0} R_{1}\left(R_{3}-R_{2}\right) .
$$

From (2.29) we find that $R_{2} \neq R_{3}$, and thanks to the definition of $R_{1}$ equation (2.49) becomes:

$$
\bar{U}_{0}^{2} f^{2} \lambda^{2} / N^{2}=\mu_{0}^{2}
$$

which contradicts (2.30). Thus the matrix $\Lambda(x)$ is regular for every $x \in \mathbb{R}$.

Back to equation (2.46), and thanks to the latter result, the functions $B_{i}^{\prime}(x)$ are uniquely determined for $i=1,2,3$. It remains to use the modal boundary conditions to determine the constants $B_{i}(0)$ and thus the functions $B_{i}(x)$.

At this point, it is desirable to reintroduce the indices $n$, i.e. to return to the notation $\left(u_{n}, v_{n}, \psi_{n}\right)$, since the boundary conditions depend on the mode considered. For the supercritical modes $\left(n>n_{c}\right)$, the modal boundary condition is that in (2.12). We thus look for the $B_{i}(0)$ satisfying:

$$
\left\{\begin{aligned}
a_{2} B_{2}(0)+a_{3} B_{3}(0) & =0 \\
B_{1}(0)+B_{2}(0)+B_{3}(0) & =0 \\
c_{1} B_{1}(0)+c_{2} B_{2}(0)+c_{3} B_{3}(0) & =0
\end{aligned}\right.
$$

The matrix of this system is again $\Lambda(0)$ which was shown to be regular (see above). We conclude that the constants $B_{i}(0)$ are uniquely 
determined by (2.51) and equal to zero. The functions $B_{i}(x)$ for the supercritical modes $\left(n>n_{c}\right)$ are now fully determined.

If $n \leq n_{c}$, the mode is subcritical and we consider the boundary condition (2.14). We thus want to solve the following system:

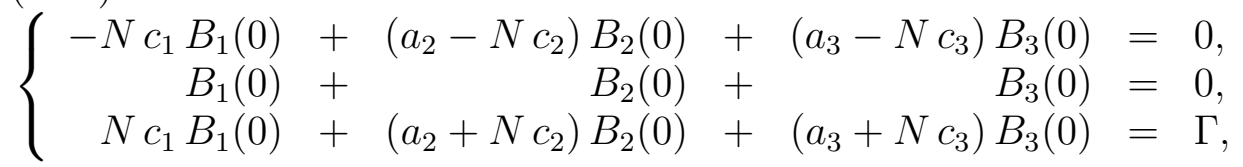

where

$$
\Gamma=-\sum_{i=1}^{3} \int_{0}^{L_{1}}\left(a_{i}+N c_{i}\right) B_{i}^{\prime}(x) d x
$$

The quantity $\Gamma$ depends only on the data and on the $B_{i}^{\prime}$, hence it is known at this stage. After some computations and using hypotheses (2.29) and (2.30), we check that the matrix of the linear system (2.52) is regular (same proof exactly as for $\Lambda(0)$ ). This achieves the determination of the $B_{i}$ in the subcritical case, and the lemma is proved.

Remark 2.3. The case when there exists $n \geq 1$ such that $\bar{U}_{0}=N / \lambda_{n}$ is slightly different and actually simpler since the third equation (2.13) becomes $\partial \eta_{n}(x, t) / \partial t=F_{\eta, n}(t)$, which can be integrated directly. We note that no boundary condition (neither in the subcritical case nor in the supercritical one) would then be required for $\eta_{n}$ so that $(2.23),(2.24)$ would have to be modified. But we do not want to go into the details since this nongeneric situation seldom occurs in numerical simulations.

To conclude there remains to verify the hypothesis $(i)$ of the HilleYosida theorem, that is:

Lemma 2.3. The domain $D(A)$ of $A$ is dense in $H$, and the operator A is closed.

Proof. We first verify that the orthogonal in $H$ of $D(A), D(A)^{\perp}$, is reduced to $\{0\}$.

Let $v$ be an element of $D(A)^{\perp}$. Since $A+\mu_{0} I$ is onto, there exists $u \in D(A)$ such that $\left(A+\mu_{0} I\right) u=v$. Then:

$$
0=(v, u)_{H}=\left(\left(A+\mu_{0} I\right) u, u\right)_{H} \geq \mu_{0}|u|_{H}^{2}
$$


hence $u=v=0$, which implies that $D(A)^{\perp}=\{0\}$, and $D(A)$ is dense in $H$.

To show that $A$ is closed, we consider a sequence $\left(u_{j}, v_{j}, \psi_{j}\right)=U_{j}$ of $D(A)$, such that :

$$
\begin{aligned}
U_{j} & \longrightarrow U \text { in } H, \\
A U_{j}=F_{j} & \longrightarrow F \text { in } H,
\end{aligned}
$$

and we want to verify that $U=(u, v, \psi) \in D(A)$ and $F=A U$, so that the graph of $A$ is closed.

Thanks to (2.54), we know that

$$
\begin{aligned}
& u_{j} \longrightarrow u \text { in } H_{u} \subset L^{2}(\mathcal{M}), \\
& v_{j} \longrightarrow v \text { in } L^{2}(\mathcal{M}) .
\end{aligned}
$$

We also find from (2.25) and (2.55) that

$$
\bar{U}_{0} \frac{\partial^{2} v_{j}}{\partial x^{2}}+f u_{j} \longrightarrow F_{2} \text { in } L^{2}(\mathcal{M})
$$

Hence the sequence $\left(\partial v_{j} / \partial x\right)_{j \in \mathbb{N}}$ is bounded in $L^{2}(\mathcal{M})$, and thanks to $(2.57)$ we obtain that $v_{x} \in L^{2}(\mathcal{M})$.

In view of proving that $\left(u_{x}, \psi_{x}\right) \in L^{2}(\mathcal{M})$, we consider the decomposition in normal modes. Thanks to (2.54), we have for every $n \geq 1$ :

$$
\begin{aligned}
u_{j, n} & \longrightarrow u_{n} \text { in } L^{2}\left(0, L_{1}\right), \\
v_{j, n} & \longrightarrow v_{n} \text { in } L^{2}\left(0, L_{1}\right), \\
\psi_{j, n} & \longrightarrow \psi_{n} \text { in } L^{2}\left(0, L_{1}\right),
\end{aligned}
$$

and the quantities $\sum_{n \geq 1}\left|u_{j n}\right|^{2}, \sum_{n \geq 1}\left|v_{j n}\right|^{2}$ and $\sum_{n \geq 1}\left|\psi_{j n}\right|^{2}$ are bounded uniformly in $j$.

Similarly, we infer from (2.55) that for every $n \geq 1$ the following convergences in $L^{2}\left(0, L_{1}\right)$ :

$$
\begin{aligned}
& \bar{U}_{0} \frac{\partial^{2} u_{j, n}}{\partial x^{2}}-f v_{n}-\frac{1}{\lambda_{n}} \frac{\partial^{2} \psi_{j, n}}{\partial x^{2}}=F_{u, n}^{j} \longrightarrow F_{u, n}, \\
& \bar{U}_{0} \frac{\partial^{2} v_{j, n}}{\partial x^{2}}+f u_{n}=F_{v, n}^{j} \longrightarrow F_{v, n}, \\
& \bar{U}_{0} \frac{\partial^{2} \psi_{j, n}}{\partial x^{2}}-\frac{N^{2}}{\lambda_{n}} \frac{\partial^{2} u_{j, n}}{\partial x^{2}}=F_{\psi, n}^{j} \longrightarrow F_{\psi, n},
\end{aligned}
$$


and the quantities $\sum_{n \geq 1}\left|F_{u, n}^{j}\right|^{2}, \sum_{n \geq 1}\left|F_{v, n}^{j}\right|^{2}$ and $\sum_{n \geq 1}\left|F_{\psi, n}^{j}\right|^{2}$ are bounded uniformly in $j$.

Combining (2.62) and (2.64), we find that:

$$
\frac{\partial^{2} u_{j, n}}{\partial x^{2}}=\frac{1}{\bar{U}_{0}^{2}-N^{2} / \lambda_{n}^{2}}\left(\bar{U}_{0} F_{u, n}^{j}+f \bar{U}_{0} v_{j, n}+\frac{F_{\psi, n}^{j}}{\lambda_{n}}\right) ;
$$

hence the $\left(\partial u_{j, n} / \partial x\right)_{j \geq 1}$ are bounded in $L^{2}\left(0, L_{1}\right)$ and $\left(d u_{n} / d x\right) \in$ $L^{2}\left(0, L_{1}\right)$. Moreover, we find that ${ }^{7}$

$\sum_{n \geq 1}\left|\frac{\partial^{2} u_{j, n}}{\partial x^{2}}\right|^{2} \leq \frac{4}{\min _{n \geq 1}\left|\bar{U}_{0}^{2}-N^{2} / \lambda_{n}^{2}\right|^{2}} \sum_{n \geq 1}\left(\bar{U}_{0}^{2}\left|F_{u, n}^{j}\right|^{2}+f^{2} \bar{U}_{0}^{2}\left|v_{j, n}\right|^{2}+\left|\frac{F_{\psi, n}^{j}}{\lambda_{n}}\right|^{2}\right)$,

so that the latter quantity is bounded uniformly in $j$. This guarantees that $u_{x} \in L^{2}(\mathcal{M})$. Following the same idea, and using either (2.62) or (2.64), we also prove that $\psi_{x} \in L^{2}(\mathcal{M})$.

To insure that $U \in D(A)$, we need to verify that the modal boundary conditions (2.12) and (2.14) are satisfied by $U$. This is clear since the convergence of $\left(u_{j, n}, v_{j, n}, \psi_{j, n}\right)$ to $\left(u_{n}, v_{n}, \psi_{n}\right)$ is in fact in $H^{1}\left(0, L_{1}\right)$, so that the boundary conditions pass to the limit.

Finally, let us show that $A U=F$. Thanks to (2.54), we find that $A U_{j} \rightarrow A U$ in the distribution sense in $\mathcal{M}$, hence $A U=F$ in the sense of distributions on $\mathcal{M}$. We infer from $U \in D(A)$ that $A U \in L^{2}(\mathcal{M})$, and conclude that $A U=F$ in $L^{2}(\mathcal{M})$, which ends the proof of Lemma 2.3 .

\subsubsection{The case of nonhomogeneous boundary conditions.}

In practical simulations, we want to solve the PEs with nonhomogeneous boundary conditions on $U$ at $x=0$ and $x=L_{1}$, that is $U$ given respectively equal to $U^{g, l}$ and $U^{g, r}$. We assume that these boundary values are derived from a solution $\widetilde{U}$ given or computed on a domain $\widetilde{\mathcal{M}}$ larger than $\mathcal{M}^{8}$.

We discussed in Section 2.3.3 above the case when $U^{g, l}=U^{g, r}=0$. The issue is now to determine which components of $U^{g, l}$ and $U^{g, r}$ are

\footnotetext{
${ }^{7}$ Thanks to (1.24), we know that $\min _{n \geq 1}\left|\bar{U}_{0}^{2}-N^{2} / \lambda_{n}^{2}\right|>0$.

${ }^{8}$ Assuming e.g. periodical boundary conditions for $\widetilde{\mathcal{M}}$.
} 
needed to obtain a well-posed problem. In this context all components of $U^{g, l}$ and $U^{g, r}$ are available but we know (or surmise at this point) that they will not be all used, those used depending on the mode that we consider.

Based on the data $U^{g, l}, U^{g, r}$, let us now construct the following function $U^{g}=\left(u^{g}, v^{g}, \psi^{g}\right)$ depending on $z$ and $t$ and defined by:

$$
\left(u^{g}, v^{g}, \psi^{g}\right)(z, t)=\sum_{n \geq 1}\left(u_{n}^{g}(t) \mathcal{U}_{n}(z), v_{n}^{g}(t) \mathcal{U}_{n}(z), \psi_{n}^{g}(t) \mathcal{W}_{n}(z)\right)
$$

where $\left(u_{n}^{g}, v_{n}^{g}, \psi_{n}^{g}\right)$ are found using the boundary values $U^{g, l}$ and $U^{g, r}$ by:

$$
\left\{\begin{aligned}
& u_{n}^{g}(t)-\frac{1}{N} \psi_{n}^{g}(t)=u_{n}^{g, l}(t)-\frac{1}{N} \psi_{n}^{g, l}(t), \\
& v_{n}^{g}(t)=v_{n}^{g, l}(t), \\
& u_{n}^{g}(t)+\frac{1}{N} \psi_{n}^{g}(t)=u_{n}^{g, r}(t)+\frac{1}{N} \psi_{n}^{g, r}(t), \\
&\left\{\begin{aligned}
u_{n}^{g}(t) & =u_{n}^{g, l}(t), \\
v_{n}^{g}(t) & =v_{n}^{g, l}(t), \quad n>n_{c} . \\
\psi_{n}^{g}(t) & =\psi_{n}^{g, l}(t),
\end{aligned}\right.
\end{aligned}\right.
$$

We note that $U^{g}$ is a function of $z$ and $t$ only, and hence it does not depend on the horizontal coordinate $x$. Setting $F^{\#}=F-d U^{g} / d t$ and $U_{0}^{\#}=U_{0}-U_{0}^{g}$ where $U_{0}^{g}=U^{g}(t=0)$, we will look for $U^{\#}$ solution of

$$
\left\{\begin{array}{l}
\frac{d U^{\#}}{d t}+A U^{\#}=F^{\#} \\
U^{\#}(t=0)=U_{0}^{\#}
\end{array}\right.
$$

Like (2.16) this equation corresponds to the case with homogeneous boundary conditions. In order to apply Theorem 2.2 to (2.70), we would need to have

$$
U_{0}^{\#}=U_{0}-U_{t=0}^{g} \in D(A)
$$

and

$$
F^{\#}, \frac{d F^{\#}}{d t} \in L^{1}(O, T ; H) .
$$

We will state in Theorem 2.4 below some assumptions on $U^{g, l}$ and $U^{g, r}$ which guarantee that $U_{0}^{\#}$ and $F^{\#}$ satisfy (2.72) and (2.71). Writting $U=U^{\#}+U^{g}$, we find that $U$ is solution of (2.1), and the boundary conditions of $U$ at $x=0$ and $x=L_{1}$ are those of $U^{g}$, that is for the subcritical modes $\left(1 \leq n \leq n_{c}\right)$ : 


$$
\left\{\begin{array}{l}
\int_{-L_{3}}^{0} u(0, z, t) \mathcal{U}_{n}(z) d z-\frac{1}{N} \int_{-L_{3}}^{0} \psi(0, z, t) \mathcal{W}_{n}(z) d z \\
=\int_{-L_{3}}^{0} u^{g, l}(z, t) \mathcal{U}_{n}(z) d z-\frac{1}{N} \int_{-L_{3}}^{0} \psi^{g, l}(z, t) \mathcal{W}_{n}(z) d z \\
\int_{-L_{3}}^{0} v(0, z, t) \mathcal{U}_{n}(z) d z=\int_{-L_{3}}^{0} v^{g, l}(z, t) \mathcal{U}_{n}(z) d z \\
\int_{-L_{3}}^{0} u\left(L_{1}, z, t\right) \mathcal{U}_{n}(z) d z+\frac{1}{N} \int_{-L_{3}}^{0} \psi\left(L_{1}, z, t\right) \mathcal{W}_{n}(z) d z \\
=\int_{-L_{3}}^{0} u^{g, r}(z, t) \mathcal{U}_{n}(z) d z+\frac{1}{N} \int_{-L_{3}}^{0} \psi^{g, r}(z, t) \mathcal{W}_{n}(z) d z
\end{array}\right.
$$

and for the supercritical ones $\left(n>n_{c}\right)$ :

$$
\left\{\begin{array}{l}
\int_{-L_{3}}^{0} u(0, z, t) \mathcal{U}_{n}(z) d z=\int_{-L_{3}}^{0} u^{g, l}(z, t) \mathcal{U}_{n}(z) d z \\
\int_{-L_{3}}^{0^{0}} v(0, z, t) \mathcal{U}_{n}(z) d z=\int_{-L_{3}}^{0} v^{g, l}(z, t) \mathcal{U}_{n}(z) d z \\
\int_{-L_{3}}^{0} \psi(0, z, t) \mathcal{W}_{n}(z) d z=\int_{-L_{3}}^{0} \psi^{g, l}(z, t) \mathcal{W}_{n}(z) d z
\end{array}\right.
$$

Thus we have established the following result:

Theorem 2.4. Let $H$ be the Hilbert space defined in (2.17) and $A$ be the linear operator defined in (2.25) corresponding to the two-dimensional linearized Primitive Equations with vanishing viscosity. We are given the boundary values $U^{g, l}$ and $U^{g, r}$ which are in $L^{1}\left(0, T ; L^{2}\left(-L_{3}, 0\right)^{3}\right)$, together with their first time derivatives, and $F$ and $F^{\prime}=d F / d t \in$ $L^{1}\left(0, T ; L^{2}(\mathcal{M})^{3}\right)$.

Then the initial value problem corresponding to equations (2.1), supplemented with the boundary conditions (2.73) and (2.74) is well-posed, that is for every initial data $U_{0} \in U_{0}^{g}+D(A)^{9}$, there exists a unique solution $U \in \mathcal{C}([0, T] ; H)$ of (2.1) verifying (2.73) and (2.74), and $U(0)=U_{0}$.

\subsubsection{The barotropic mode.}

We now return to the mode constant in $z$, when $n=0$. This mode does not raise any mathematical difficulty, but it is fundamental in the

\footnotetext{
${ }^{9}$ This means that $U_{0}$ has the same smoothness as a function of $D(A)$ and (2.73),(2.74) are satisfied at $t=0$.
} 
numerical simulations, since it carries much energy.

Integrating $(2.5 \mathrm{a}),(2.5 \mathrm{~b})$, and $(2.5 \mathrm{e})$ on $\left(-L_{3}, 0\right)$ we find:

$$
\begin{aligned}
\frac{\partial u_{0}}{\partial t}+\bar{U}_{0} \frac{\partial u_{0}}{\partial x}-f v_{0}+\frac{\partial \phi_{0}}{\partial x} & =F_{u, 0}, \\
\frac{\partial v_{0}}{\partial t}+\bar{U}_{0} \frac{\partial v_{0}}{\partial x}+f u_{0} & =F_{v, 0}, \\
\frac{\partial u_{0}}{\partial x} & =0 .
\end{aligned}
$$

We propose to supplement this system with the following boundary conditions:

$$
\begin{aligned}
& u_{0}(0, t)=u_{l}(t), \\
& v_{0}(0, t)=v_{l}(t),
\end{aligned}
$$

with $u_{l}, v_{l}$ given (not necessarily zero, as in Section 2.3.4).

Then, since $\partial u_{0} / \partial x=0, u_{0}$ does not depend on $\mathrm{x}$, and it is thus equal to $u_{l}(t)$ everywhere, so that $(2.78)$ means in fact that

$$
u_{0}(x, t)=u_{l}(t), \quad \forall(x, t) \in\left(0, L_{1}\right) \times \mathbb{R}_{+}^{*} .
$$

Introducing (2.80) in (2.76), we find that:

$$
\frac{\partial v_{0}}{\partial t}+\bar{U}_{0} \frac{\partial v_{0}}{\partial x}=F_{v, 0}-f\left(\bar{U}_{0}+u_{l}\right)
$$

When we supplement (2.81) with the boundary condition (2.79), we have a simple well-posed problem and $v_{0}$ is given in terms of the data by integration along the characteristics.

Finally, once both $u_{0}$ and $v_{0}$ are known, equation (2.75) gives $\phi_{0}$, up to an additive constant (as expected):

$$
\begin{aligned}
\phi_{0}(x, t) & =\phi_{0}(0, t)+\int_{0}^{x}\left\{f v_{0}\left(x^{\prime}, t\right)-\frac{\partial u_{0}}{\partial t}\left(x^{\prime}, t\right)\right\} d x^{\prime} \\
& =\phi_{0}(0, t)-x u_{l}^{\prime}(t)+f \int_{0}^{x} v_{0}\left(x^{\prime}, t\right) d x^{\prime}
\end{aligned}
$$

2.4. Numerical Simulations. In this section and the next one, we describe the numerical simulations performed in the 2D linear and nonlinear cases. We start by presenting the numerical scheme. 
2.4.1. Vertical Decomposition. In the vertical direction, we proceed by normal modes decomposition as in (2.6), (2.7). From the numerical point of view, we will need to transform some grid-data into modal coefficients in the $\mathcal{U}_{m}$ or $\mathcal{W}_{m}$ bases of $L^{2}\left(-L_{3}, 0\right)$, and vice versa, using Fourier and inverse Fourier transforms for example.

Given a function $f$ represented by its values $f_{l}$ on a grid $z_{l}=-L_{3}+l \Delta z$, $0 \leq l \leq l_{\max }, \Delta z=L_{3} / l_{\max }$, we want to find its coefficients $f_{m}$ in the modal decomposition (2.6)-(2.7), limited to $0 \leq m \leq M$. To this aim we use the second order central point integration method, with the $z_{l}$ as collocation points. For the functions $u, v$ and $\phi$, we decompose them in the $\mathcal{U}_{m}$ basis of $L^{2}\left(-L_{3}, 0\right)$. For $0 \leq m \leq M$ :

$\left\{u_{m}, v_{m}, \phi_{m}\right\}=\Delta z \sum_{l=0}^{l_{\max }-1} \frac{\mathcal{U}_{m}\left(z_{l}\right) \cdot\{u, v, \phi\}\left(z_{l}\right)+\mathcal{U}_{m}\left(z_{l+1}\right) \cdot\{u, v, \phi\}\left(z_{l+1}\right)}{2}$,

and for $w$ and $\psi, 1 \leq m \leq M$ :

$$
\left\{w_{m}, \psi_{m}\right\}=\Delta z \sum_{l=0}^{l_{\max }-1} \frac{\mathcal{W}_{m}\left(z_{l}\right) \cdot\{w, \psi\}\left(z_{l}\right)+\mathcal{W}_{m}\left(z_{l+1}\right) \cdot\{w, \psi\}\left(z_{l+1}\right)}{2} .
$$

This approach which is that proposed by the physicists is different from the more mathematical approach to spectral and pseudo-spectral methods as in e.g. Bernardi and Maday (1997), Gottlieb and Hesthaven (2001). The advantage of such a choice is that the orthogonality relations (see Section1.2) are satisfied from the numerical point of view. Further studies and comparisons of the two approaches will be needed in the future.

On the contrary, if the function is given by its modal coefficients, the values on the $z$-grid $z_{l}, 0 \leq l \leq l_{\max }$ is simply given by:

$$
\begin{array}{r}
(u, v, \phi)\left(z_{l}\right)=\sum_{m=0}^{M}\left(u_{m}, v_{m}, \phi_{m}\right) \mathcal{U}_{m}\left(z_{l}\right), \\
(w, \psi)\left(z_{l}\right)=\sum_{m=0}^{M}\left(w_{m}, \psi_{m}\right) \mathcal{W}_{m}\left(z_{l}\right) .
\end{array}
$$

In the numerical simulations, we are given some initial data on the physical grid $\left(z_{l}\right)_{0 \leq l \leq l_{\max }}$. We transform them into modal coefficients thanks to formulas $(2.83)$ or $(2.84)$, and if the problem is linear, we 
keep them all along the computations, except for graphic purposes, for which we use the inverse formulas (2.85)-(2.86) to return to the physical space. Naturally, in the nonlinear case, we will need to operate (2.83)(2.86) once at every time step, in order to avoid the computation of a convolution product, that would cost too much in term of CPU time and is not considered an appropriate (stable) numerical procedure. We compute the nonlinear terms of the equations in the physical space $(x, z)$ thanks to Fourier and inverse Fourier transforms.

2.4.2. Finite Differences in Time and Space. Looking at the form of (2.13), we choose to discretize these equations in the horizontal direction with a finite differences method. Naturally, care has to be taken to the sign of the characteristic values, in order to take an upwind (hence stable) spatial discretization of the $x$-derivative. Whereas $\bar{U}_{0}$ and $\bar{U}_{0}+N / \lambda_{m}$ are always positive, the third characteristic value of the $m t h$ mode, in the linear case, is $\bar{U}_{0}-N / \lambda_{m}$ and can either be positive or negative for the actual physical values that we consider.

With this in mind, for every subcritical mode $m \leq n_{c}$, we discretize (2.15) as follows:

$$
\frac{\xi_{m, j}^{n+1}-\xi_{m, j}^{n}}{\Delta t^{n}}+\left(\bar{U}_{0}+\frac{N}{\lambda_{m}}\right) \frac{\xi_{m, j}^{n}-\xi_{m, j-1}^{n}}{\Delta x}-f v_{m, j}^{n}=F_{\xi, m, j}^{n}-B_{\xi, m, j}^{n},
$$

$$
\frac{v_{m, j}^{n+1}-v_{m, j}^{n}}{\Delta t^{n}}+\bar{U}_{0} \frac{v_{m, j}^{n}-v_{m, j-1}^{n}}{\Delta x}+f \frac{\xi_{m, j}^{n}+\eta_{m, j}^{n}}{2}=F_{v, m, j}^{n}-B_{v, m, j}^{n},
$$

$$
\frac{\eta_{m, j}^{n+1}-\eta_{m, j}^{n}}{\Delta t^{n}}+\left(\bar{U}_{0}-\frac{N}{\lambda_{m}}\right) \frac{\eta_{m, j+1}^{n}-\eta_{m, j}^{n}}{\Delta x}-f v_{m, j}^{n}=F_{\eta, m, j}^{n}-B_{\eta, m, j}^{n} .
$$

where the right-hand-side of (2.87) contains the nonlinear terms, computed explicitly thanks to an Adams-Bashforth scheme.

Equations (2.87a) and(2.87b) hold for $1 \leq j \leq J$, whereas (2.87c) is written for $0 \leq j \leq J-1$. There are no equations for $\xi_{m, 0}^{n+1}, v_{m, 0}^{n+1}$ and $\eta_{m, J}^{n+1}$, these quantities being given by the boundary conditions as required in $(2.23),(2.24)$, see also Rousseau, Temam, and Tribbia (2005b). On the contrary, if $m>n_{c}$ (supercritical case), we propose 
for $1 \leq j \leq J$ the discretized equations

(2.88a)

$$
\frac{\xi_{m, j}^{n+1}-\xi_{m, j}^{n}}{\Delta t^{n}}+\left(\bar{U}_{0}+\frac{N}{\lambda_{m}}\right) \frac{\xi_{m, j}^{n}-\xi_{m, j-1}^{n}}{\Delta x}-f v_{m, j}^{n}=F_{\xi, m, j}^{n}-B_{\xi, m, j}^{n},
$$

$$
\frac{v_{m, j}^{n+1}-v_{m, j}^{n}}{\Delta t^{n}}+\bar{U}_{0} \frac{v_{m, j}^{n}-v_{m, j-1}^{n}}{\Delta x}+f \frac{\xi_{m, j}^{n}+\eta_{m, j}^{n}}{2}=F_{v, m, j}^{n}-B_{v, m, j}^{n},
$$

$$
\frac{\eta_{m, j}^{n+1}-\eta_{m, j}^{n}}{\Delta t^{n}}+\left(\bar{U}_{0}-\frac{N}{\lambda_{m}}\right) \frac{\eta_{m, j}^{n}-\eta_{m, j-1}^{n}}{\Delta x}-f v_{m, j}^{n}=F_{\eta, m, j}^{n}-B_{\eta, m, j}^{n} .
$$

Either $\xi_{m, 0}^{n+1}, v_{m, 0}^{n+1}$ and $\eta_{m, 0}^{n+1}$ are given by the boundary conditions defined as in (2.23), (2.24) (see also Rousseau, Temam, and Tribbia (2005b), transparent boundary conditions case), or they satisfy the periodicity conditions (2.95) below (periodical case).

For every function $f(x, z, t), f_{m, j}^{n}$ represents $f_{m}\left(x_{j}, t_{n}\right)$ for $0 \leq j \leq J$, $0 \leq n \leq n_{\max }$, with

$$
\begin{array}{r}
0=x_{0}<x_{1}<\ldots<x_{j}<\ldots<x_{J}=L, \\
0=t_{0}<t_{1}<\ldots<t_{n}<\ldots<t_{n_{\max }}=T \\
\Delta x=x_{j+1}-x_{j}=\frac{L}{J}, \\
\Delta t^{n}=t_{n+1}-t_{n} . n
\end{array}
$$

In the numerical experiments, we choose an homogeneous space discretization $(\Delta x=$ const $=L / J)$. For the sake of simplicity, we choose an explicit time-scheme, with a constant time-step $\Delta t$, which will be restricted by the well-known CFL condition to guarantee stability in the linear case:

$$
\Delta t \leq \frac{\Delta x}{\max _{1 \leq m \leq M}\left(\bar{U}_{0}, \bar{U}_{0}+\frac{N}{\lambda_{m}},\left|\bar{U}_{0}-\frac{N}{\lambda_{m}}\right|\right)}=\frac{\Delta x}{\bar{U}_{0}+\frac{N}{\lambda_{1}}} .
$$

When the equations are nonlinear, the characteristic values depend on time since $\bar{U}_{0}$ has to be replaced by $u+\bar{U}_{0}$, but we assume that the initial data is such that $\left|u_{0}\right|<<\bar{U}_{0}$, which is physically relevant (Temam and Tribbia (2003)). We actually base our computations on the data in the already quoted article Temam and Tribbia (2003), that is the initial data is such that the ratio between the perturbation and the reference flow $\bar{U}_{0} \mathbf{e}_{\mathbf{x}}$ is less than $10 \%$, which is physically relevant. In the case of numerical simulations with periodic boundary conditions, 
we multiply the initial data of Temam and Tribbia (2003) by $\sin (\pi x / L)$ to make it periodic and avoid any boundary layer at $t=0$.

2.5. Numerical Results. We present hereafter two different sets of numerical results. In Section 2.5.1 we resolve the nonlinear PEs in a domain $\Omega_{0}=(0, L) \times\left(-L_{3}, 0\right)$ with periodic boundary conditions in the horizontal $(x)$ direction, and $w=0$ at $z=-L_{3}, 0$. These numerical results will provide the boundary conditions needed for the computations of Section 2.5.2 below. In Section 2.5.3 the transparent properties of the boundary conditions are introduced, and we compare the solutions computed in Section 2.5.1 and those of Section 2.5.2; this allows us to confirm the computational suitability and relevance of the boundary conditions that we have introduced.

The computations are done as follows. We fix $M$ (the chosen number of modes) and compute $\left(u_{m}^{0}, v_{m}^{0}, \psi_{m}^{0}\right)_{0<m \leq M}$ from the given data $u^{0}, v^{0}, \psi^{0}$ thanks to $(2.83)-(2.84)$.

Then, for every mode $m \leq M$, we consider the modal equations (2.15) and their discretization (2.87)-(2.88), and supplement them with the appropriate boundary conditions, either (2.96) for the periodical case or (2.100)-(2.101) for the case of transparent boundary conditions. We recall here that for every $m,\left(\xi_{m}, \eta_{m}\right)=\left(u_{m}-N \psi_{m} / \lambda_{m}, u_{m}+\right.$ $\left.N \psi_{m} / \lambda_{m}\right)$ will be the numerical unknowns to be computed, so that $\left(u_{m}, w_{m}, \psi_{m}, \phi_{m}\right)$ can be obtained with

$$
\begin{array}{r}
u_{m}(x, t)=\frac{\xi_{m}+\eta_{m}}{2}(x, t), \\
w_{m}(x, t)=-\frac{u_{m_{x}}}{\lambda_{m}}(x, t), \\
\psi_{m}(x, t)=\frac{N\left(\eta_{m}-\xi_{m}\right)}{2}(x, t), \\
\phi_{m}(x, t)=-\frac{\psi_{m}}{\lambda_{m}}(x, t) .
\end{array}
$$

As a consequence, we will only consider the quantities $\left(\xi_{m}, v_{m}, \eta_{m}\right)$ in the sequel, the other physical quantities beeing easily computed thanks to $(2.94)$.

2.5.1. Periodic boundary conditions for the large domain $\Omega_{0}$. In the periodical case, we consider the following modal boundary conditions: 


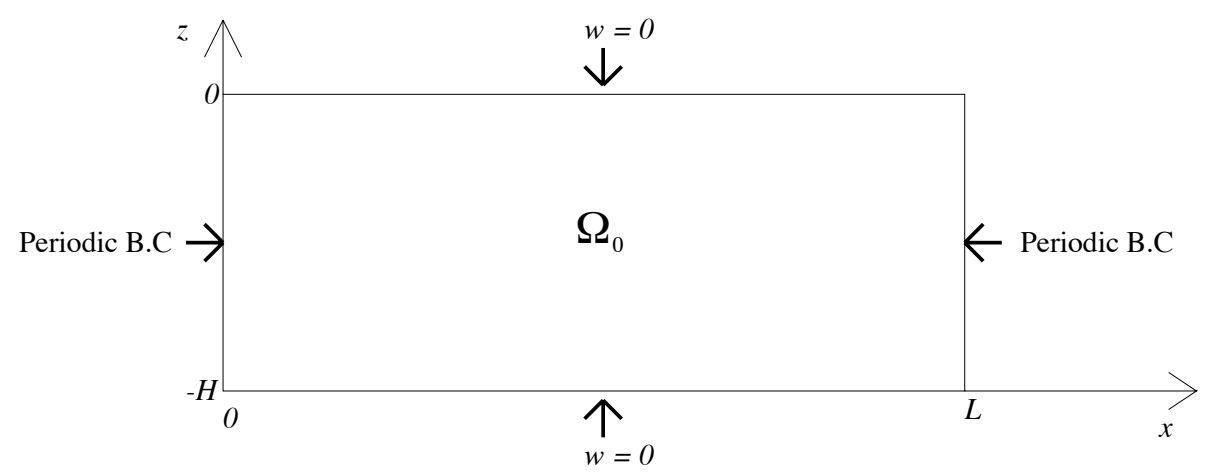

FiguRe 1. Domain $\Omega_{0}$

$$
\begin{aligned}
& \xi_{m}(0, t)=\xi_{m}(L, t), \\
& v_{m}(0, t)=v_{m}(L, t), \\
& \eta_{m}(0, t)=\eta_{m}(L, t) .
\end{aligned}
$$

For each time step $\Delta t^{n}=\Delta t$ satisfying (2.93) we compute the unknown functions $\left(\xi_{m}^{n+1}, v_{m}^{n+1}, \eta_{m}^{n+1}\right)$ thanks to (2.87) and (2.88), with the numerical boundary conditions:

$$
\begin{aligned}
& \xi_{m, 0}^{n+1}=\xi_{m, J}^{n+1}, \\
& v_{m, 0}^{n+1}=v_{m, J}^{n+1}, \\
& \eta_{m, 0}^{n+1}=\eta_{m, J}^{n+1} .
\end{aligned}
$$

The following figures plot $u, v$ and $\psi$ in the domain $\Omega_{0}$ at two different times. Figures 2, 3 and 4 represent the initial data $(t=0)$ for these three quantities, whereas Figures 5,6 and 7represent $u, v$ and $\psi$ at $t=t_{1}>0$. 

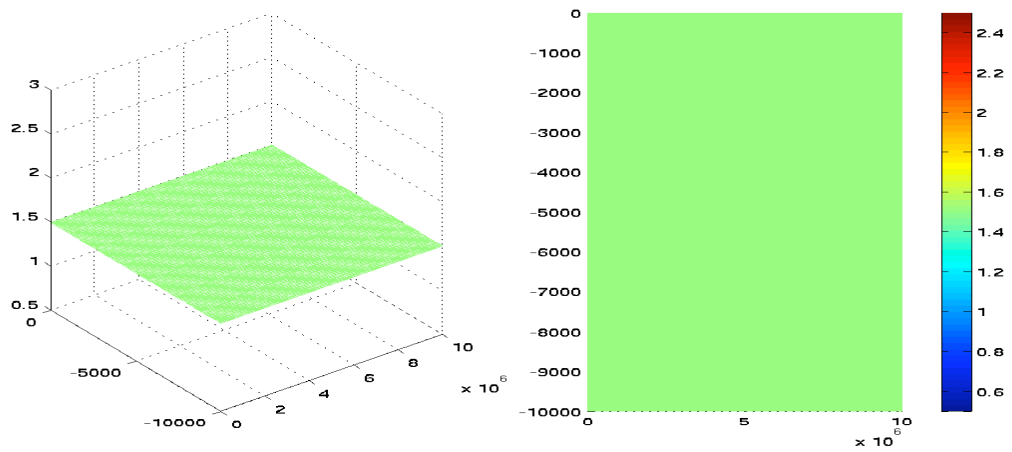

Figure 2. Periodic Boundary Condition. Initial data $u_{0}$.

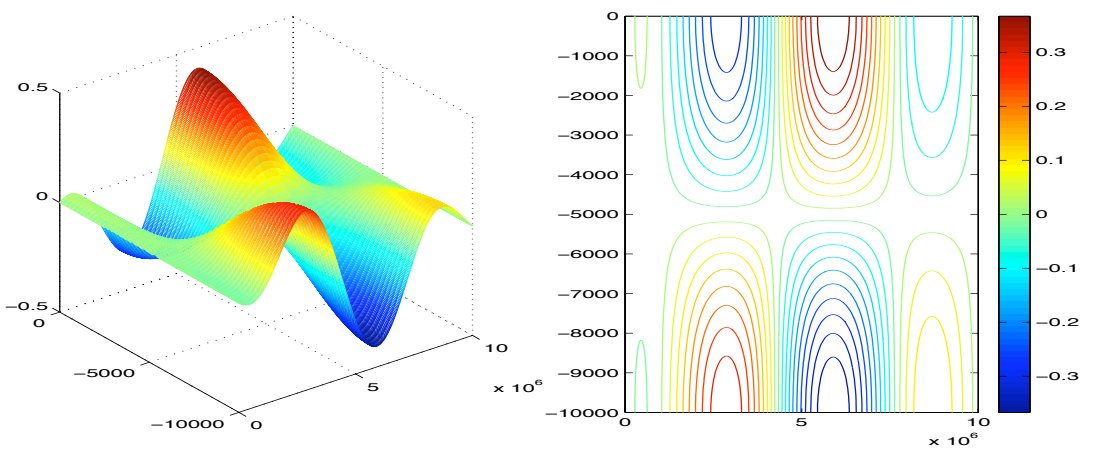

Figure 3. Periodic Boundary Condition. Initial data $v_{0}$.

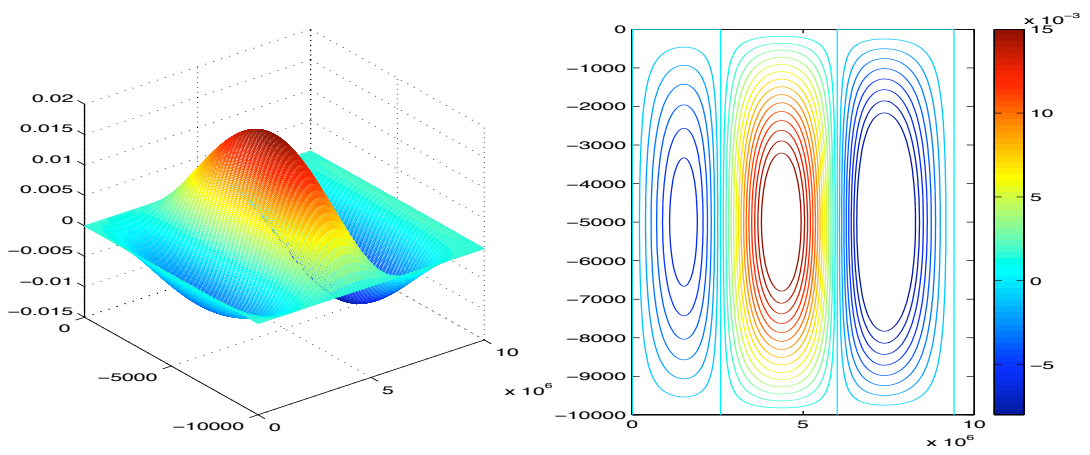

Figure 4. Periodic Boundary Condition. Initial data $\psi_{0}$. 


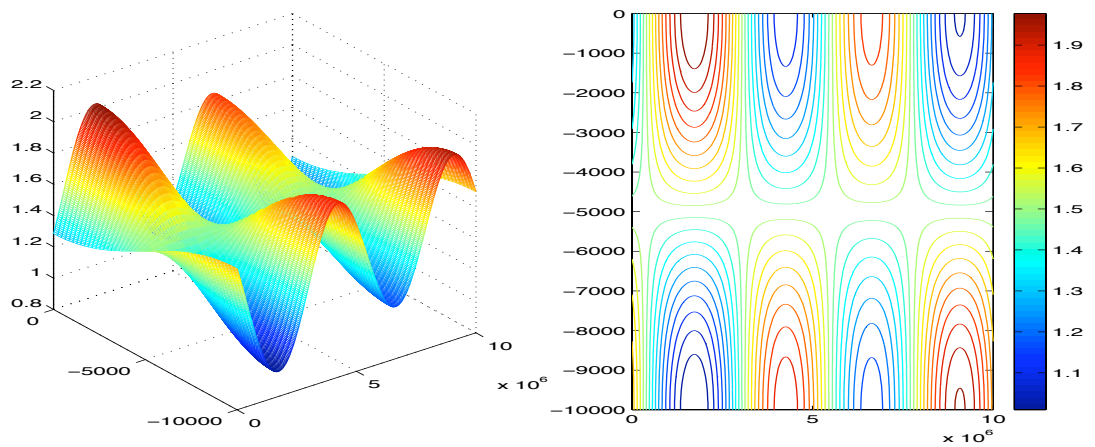

Figure 5. Periodic Boundary Condition. Values of $u$ at $t=t_{1}$.

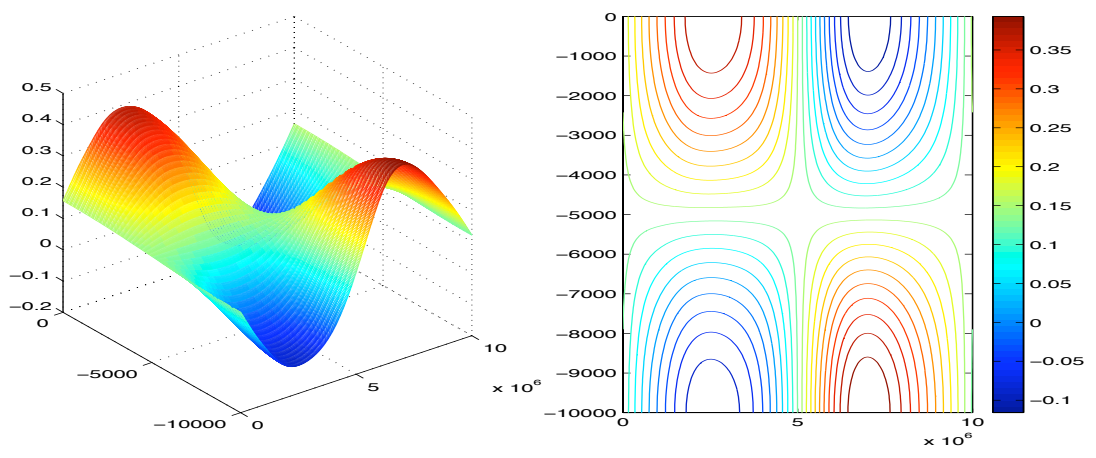

Figure 6. Periodic Boundary Condition. Values of $v$ at $t=t_{1}$.

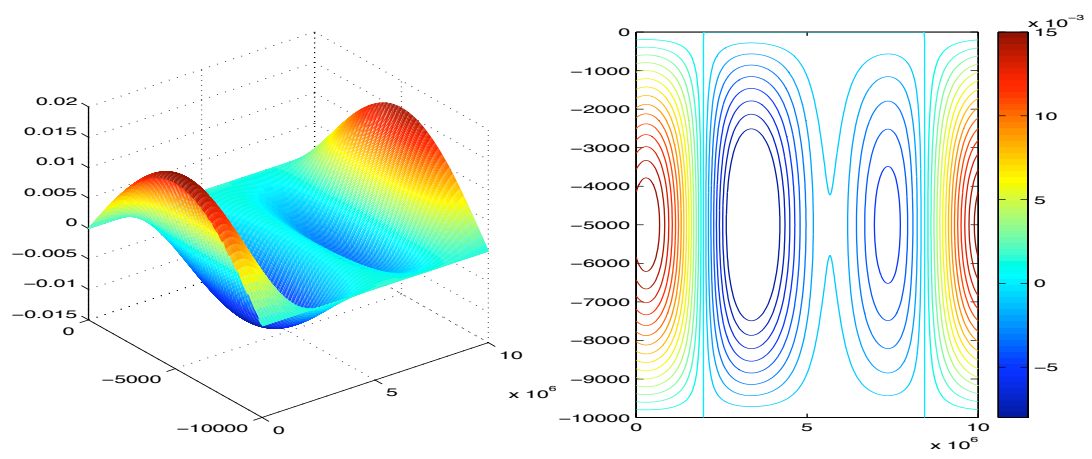

Figure 7. Periodic Boundary Condition. Values of $\psi$ at $t=t_{1}$. 


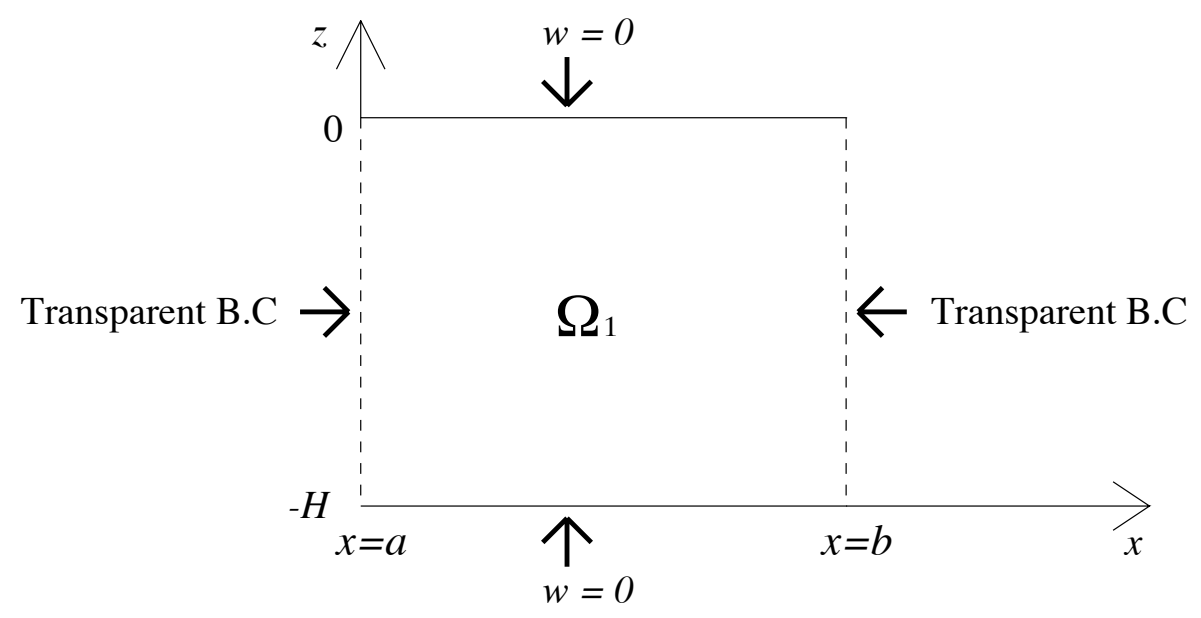

FiguRE 8. Subdomain $\Omega_{1}$

2.5.2. Transparent boundary conditions for the subdomain $\Omega_{1} \subset \Omega_{0}$. We now intend to simulate the PEs in the subdomain $\Omega_{1}=(a, b) \times$ $\left(-L_{3}, 0\right)$, and the boundary conditions at $x=a$ and $b$ are those considered in the linear case, see (2.23) and (2.24); see also Rousseau, Temam, and Tribbia (2005b). In the numerical simulations below we will consider a domain $\Omega_{1}=(a, b) \times\left(-L_{3}, 0\right)$ such that $0<a<b<L$, so that $\Omega_{1}$ is fully imbedded in $\Omega_{0}=(0, L) \times\left(-L_{3}, 0\right)$. The space discretization points are now changed to $x_{j}=a+j(b-a) / J, 0 \leq j \leq J$. At the boundaries $x=a$ and $x=b$, we will consider the nonhomogeneous form of the transparent boundary conditions of (2.23), (2.24). We use the computations of Section 2.5.1 above to provide the righthand-side of the boundary conditions (2.98) and (2.99) below, and afterwards use them for comparison in the whole subdomain $\Omega_{1}$. These boundary conditions, expressed in a general way, are given in (2.23), (2.24). They consist in an infinite set of integral boundary conditions. For example:

$$
\int_{-L_{3}}^{0} v(a, z, t) \mathcal{U}_{m}(z) d z=\int_{-L_{3}}^{0} \widetilde{v}(a, z, t) \mathcal{U}_{m}(z) d z, \quad \forall m \leq M
$$

where $\widetilde{U}=(\widetilde{u}, \widetilde{v}, \widetilde{w}, \widetilde{\psi}, \widetilde{\phi})$ are known functions, computed in the domain $\Omega_{0}$ using the periodic boundary conditions (see Section 2.5.1 above). Hence, for every subcritical mode $\left(m \leq m_{c}\right)$ and every time $t>0$, we 
have:

$$
\begin{aligned}
\xi_{m}(a, t) & =\widetilde{\xi}_{m}(a, t), \\
v_{m}(a, t) & =\widetilde{v}_{m}(a, t), \\
\eta_{m}(b, t) & =\widetilde{\eta}_{m}(b, t),
\end{aligned}
$$

where $\widetilde{\xi}_{m}$ and $\widetilde{\eta}_{m}$ are defined as usual.

For the supercritical modes, we set for every $t>0$ :

$$
\begin{aligned}
\xi_{m}(a, t) & =\widetilde{\xi}_{m}(a, t), \\
v_{m}(a, t) & =\widetilde{v}_{m}(a, t), \\
\eta_{m}(a, t) & =\widetilde{\eta}_{m}(a, t) .
\end{aligned}
$$

To implement these boundary conditions, we discretize equations (2.15) with the finite differences method, taking into account the sign of $\bar{U}_{0}-$ $N / \lambda_{m}$ for the discretization of the first $x$-derivative of $\eta_{m}$ in equation (2.15c) (see equations (2.87) and (2.88) of Section 2.2 above).

For each time step $\Delta t^{n}=\Delta t$ satisfying (2.93) we compute the unknown functions $\left(\xi_{m}^{n+1}, v_{m}^{n+1}, \eta_{m}^{n+1}\right)$ thanks to (2.87) and (2.88), with the numerical boundary conditions:

$$
\begin{aligned}
\xi_{m, 0}^{n+1} & =\widetilde{\xi}_{m}\left(a, t_{n+1}\right), \\
v_{m, 0}^{n+1} & =\widetilde{v}_{m}\left(a, t_{n+1}\right), \\
\eta_{m, J}^{n+1} & =\widetilde{\eta}_{m}\left(b, t_{n+1}\right),
\end{aligned}
$$

if $m$ is subcritical $\left(m \leq m_{c}\right)$. If $m$ is supercritical $\left(m>m_{c}\right)$, we set

$$
\begin{aligned}
\xi_{m, 0}^{n+1} & =\widetilde{\xi}_{m}\left(a, t_{n+1}\right), \\
v_{m, 0}^{n+1} & =\widetilde{v}_{m}\left(a, t_{n+1}\right), \\
\eta_{m, 0}^{n+1} & =\widetilde{\eta}_{m}\left(a, t_{n+1}\right) .
\end{aligned}
$$

The following figures plot $u, v$ and $\psi$ in the domain $\Omega_{1}$ at two different times. Figures 9, 10 and 11 represent the initial data $(t=0)$ for these three quantities, whereas Figures 12, 13 and 14 represent $u, v$ and $\psi$ at $t=t_{1}>0$.

Here, one can see that Figures 12, 13 and 14 respectively match well with Figures 5, 6 and 7 in the domain $\Omega_{1}$. 

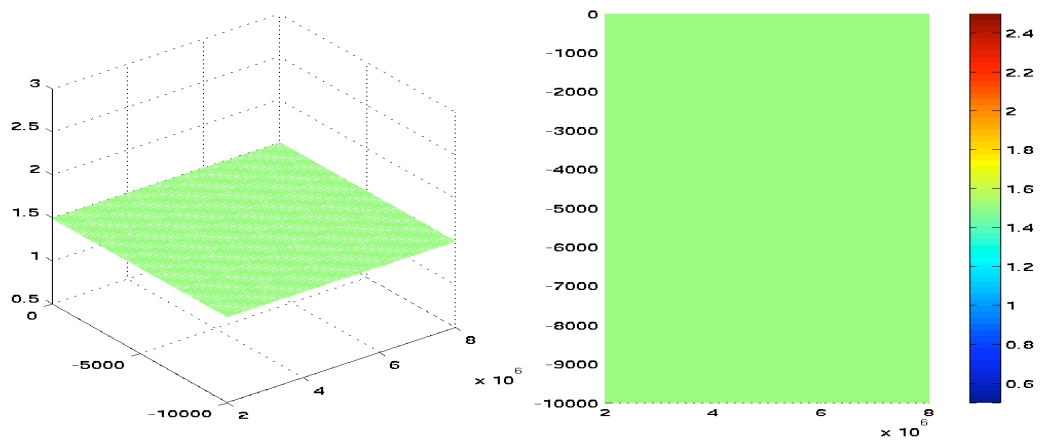

FiguRE 9. Transparent Boundary Condition. Initial data $u_{0}$.
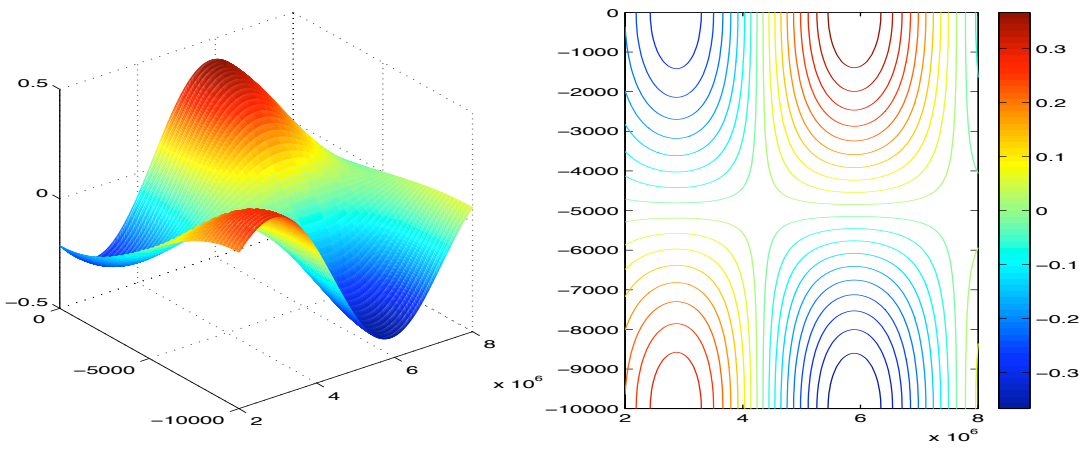

Figure 10. Transparent Boundary Condition. Initial data $v_{0}$.

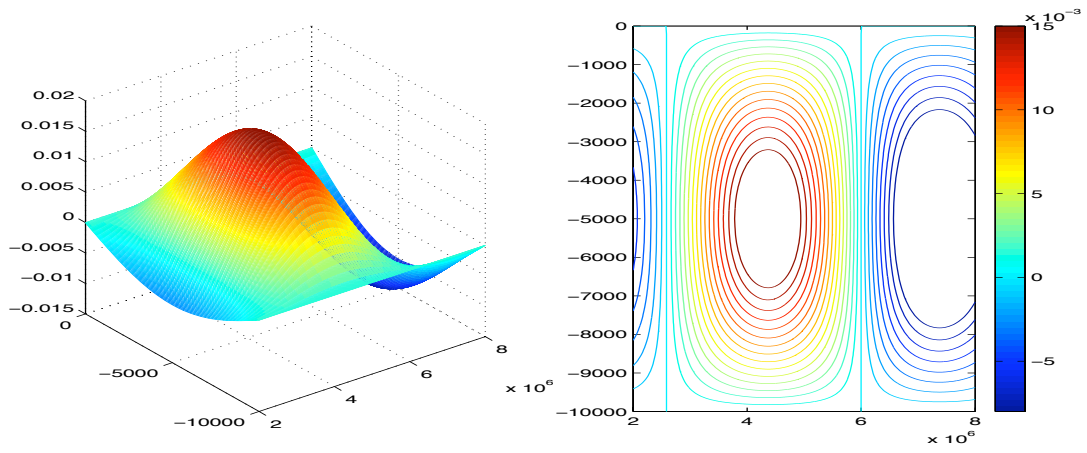

Figure 11. Transparent Boundary Condition. Initial data $\psi_{0}$. 

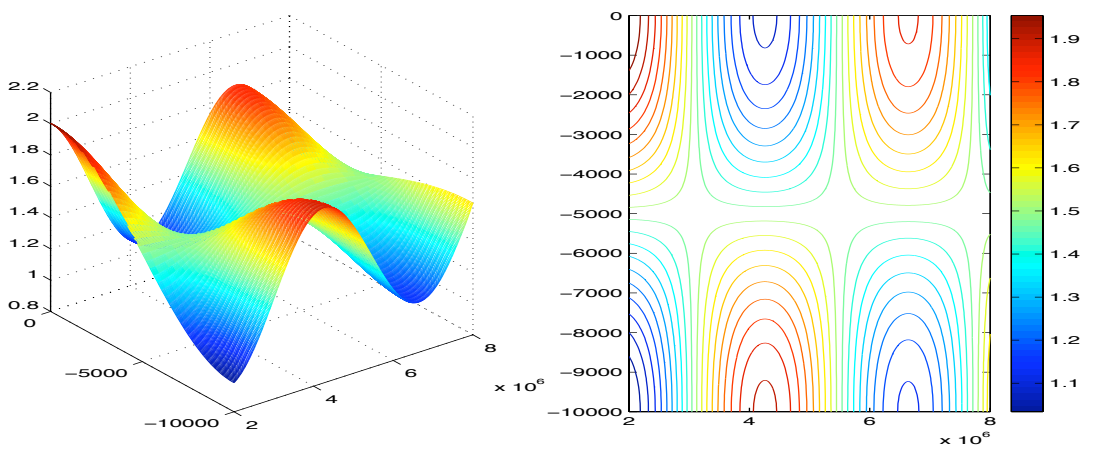

FiguRE 12. Transparent Boundary Condition. Values of $u$ at $t=t_{1}$.

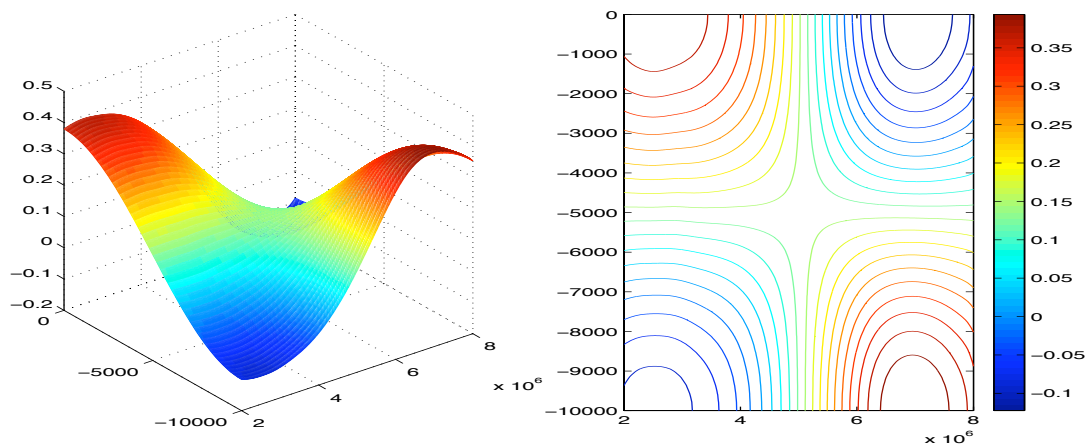

Figure 13. Transparent Boundary Condition. Values of $v$ at $t=t_{1}$.
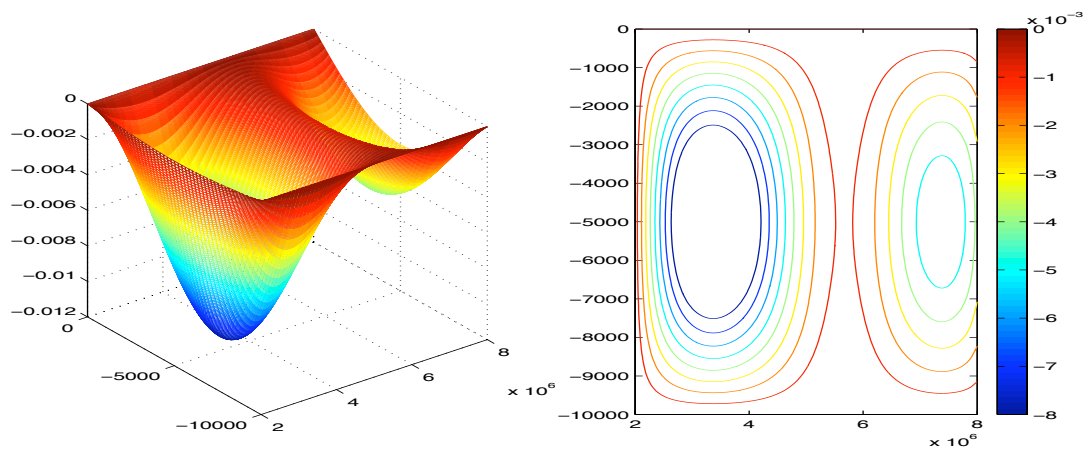

Figure 14. Transparent Boundary Condition. Values of $\psi$ at $t=t_{1}$. 


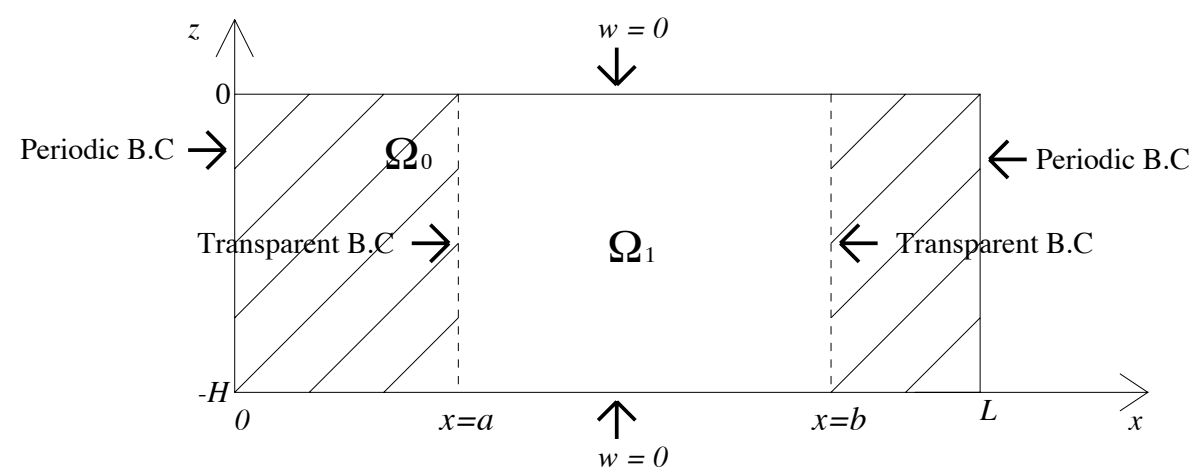

FiguRE 15. Subdomains $\Omega_{0}$ and $\Omega_{1}$

2.5.3. Comparisons. In order to quantitatively confirm what can be observed, we finally choose an interior point $\left(x_{0}, z_{0}\right)=\left(5.8 \times 10^{6},-4.0 \times\right.$ $\left.10^{3}\right) \in \Omega_{1}$, and plot in Figure 16 the values of $(u, v, \psi)\left(x_{0}, z_{0}, t\right)$ computed in $\Omega_{1}$ with transparent boundary conditions, compared to the same quantities computed in $\Omega_{0}$ with periodic boundary conditions. The results are similar if one considers another choice of $\left(x_{0}, z_{0}\right)$; this shows the transparency property of the boundary conditions (2.98)(2.99).

In the left column of Figure 16, we plot $(u, v, \psi)\left(x_{0}, z_{0}, t\right)$, these quantities beeing computed with the two types of boundary conditions. In the right column, we plot the corresponding relative errors $\left|f_{\Omega_{0}}-f_{\Omega_{1}}\right| /\left|f_{\Omega_{0}}\right|$ where $f$ is successively $u, v$ and $\psi$. The reader might think that the relative error reaches some local high values, but this is actually due to the fact that the quantity $u_{\Omega_{0}}$ (or $v_{\Omega_{0}}, \psi_{\Omega_{0}}$ ) vanishes; these local maximum are not meaningful.

\section{Space Dimension 2.5}

3.1. Motivations. We now pursue our study with a simplified 3D case, in which we allow the unknown functions to mildly depend on the $y$ variable. This case, whose motivations are given below, is called the 2.5 dimension case.

The numerical simulations performed in Section 2 were mainly motivated by computational preoccupations and the need to support the idea that the proposed boundary conditions are computationally feasible and lead indeed to well-posedness. In view of performing (in dimension two) computations of physical significance, the last author expressed the wish that the flow were a perturbation of a geostrophic 

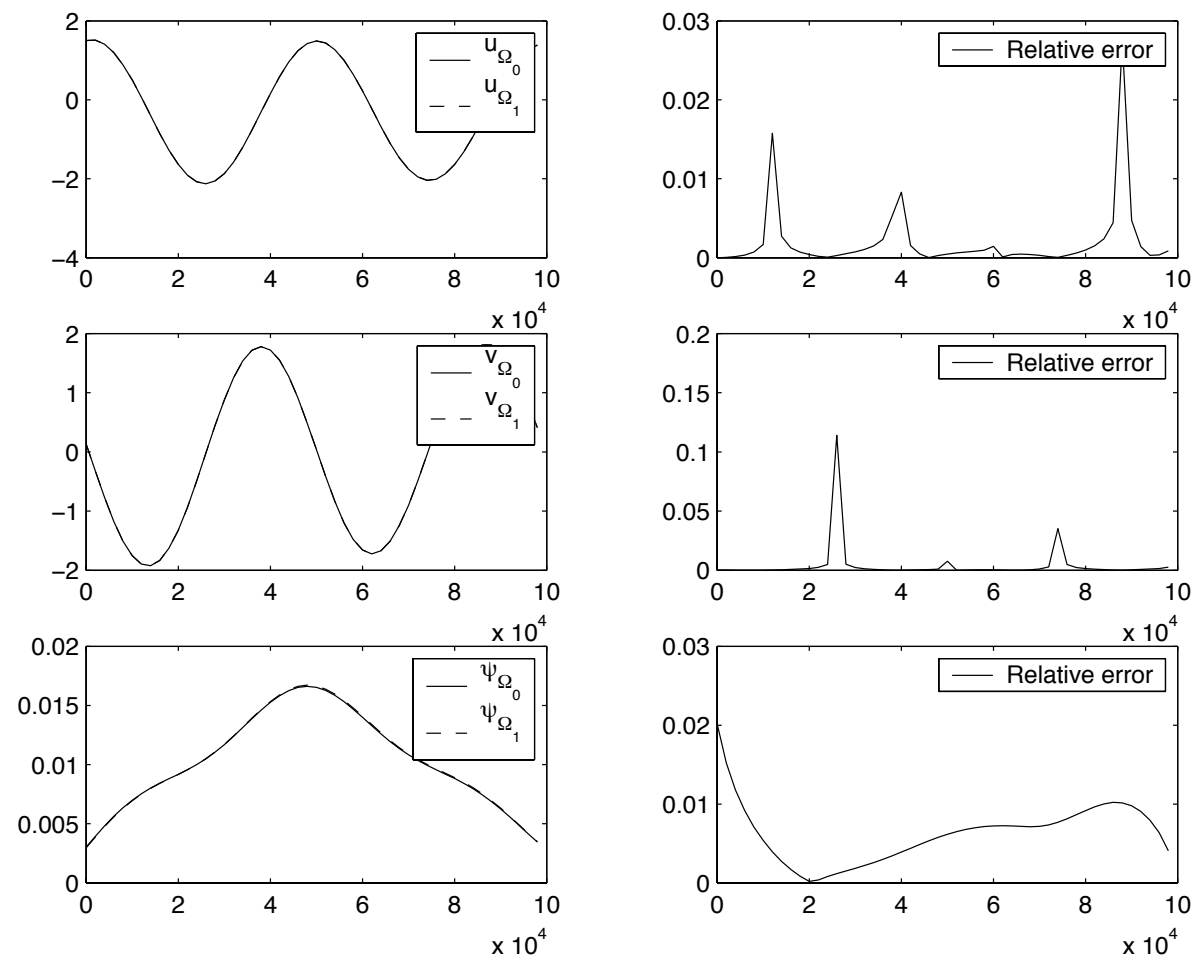

Figure 16. Computations of $(u, v, \psi)\left(x_{0}, z_{0}, t\right)$ with two different types of boundary conditions (left). Relative errors (right).

flow (which is not the case in Section 2). Now, the geostrophic equation

$$
p_{y}=-\rho f u,
$$

implies that there does not exist any geostrophic solution depending only on $x$ and $z^{10}$. In this context it is then necessary, even in dimension two, to introduce some $y$-dependence. A number of natural choices had to be abandoned, in particular the use of a few Fourier modes in $y$ for a Lorentz type model. Indeed this model would produce undesirable Gibbs phenomena when we approximate the periodic extension of the function $\sigma(y)=y$ on $\left[0, L_{2}\right]$, this function being introduced in the model by (3.1). In this way we were led to choose, for the $y$-direction, a three-mode linear finite element model. In this section, we present the full derivation of the model and study the well-posedness of the linearized equations, leaving for further studies the nonlinear case and the numerical studies.

\footnotetext{
${ }^{10} O x$ is the local west-east direction, $O y$ is the local south-north direction, and $O z$ is the ascendant vertical.
} 
This section is organized as follows. The model is derived in Section 3.2. We first derive the Galerkin finite element approximation based on the use of three piecewise linear elements in the direction $y$; we thus arrive at three coupled systems, each one similar to the 2D primitive equations in the variables $x$ and $z$ (and $t$ ). We then perform the normal mode decomposition of these equations in the direction $z$ as in Section 2, the normal modes in $z$ being either sines or cosines (depending on the functions), and these sines and cosines are the eigenfunctions of the two-point boundary value Sturm-Liouville problem (1.16)-(1.17) (Temam and Tribbia (2003)). At this stage, each mode consists of three coupled equations for the functions of the variables $x$ and $t$ (Section 3.2.2). We finally introduce, in Section 3.2.3, the boundary conditions for the latest systems in $x$ and $t$, the boundary conditions depending on the nature of the mode (subcritical or supercritical), the subcritical modes being the mathematically most challenging and physically most relevant ones. In Section 3.3 the objectives are as follows: we first establish, in the absence of the zero mode, the well-posedness of the linearized PEs, all the non-zero modes taken into account. We then pay special attention to the mode zero (barotropic part), and finally consider the case of nonhomogeneous boundary conditions; we classically reduce this case to the homogeneous case by homogenization of the boundary conditions. We consider in Section 3.4 a related model, physically interesting but with fewer degrees of freedom. The well-posedness of this model is addressed in a similar way as in Sections 3.2 and 3.3 for the first model. For this model we only emphasize the parts of the proof and the discussions which are different from the first model. The actual numerical simulations will be performed and discussed in a separate work.

3.2. The 2.5D Primitive Equations. We rewrite the 3D primitive equations for the ocean and the atmosphere without viscosity with the same notations as in (1.1)

$$
\left\{\begin{array}{l}
\widetilde{\mathbf{v}}_{t}+(\widetilde{\mathbf{v}} \cdot \nabla) \widetilde{\mathbf{v}}+\widetilde{w} \mathbf{v}_{z}+f k \times \widetilde{\mathbf{v}}+\frac{1}{\rho_{0}} \nabla \widetilde{p}=F_{\widetilde{\mathbf{v}}}, \\
\widetilde{p}_{z}=-\widetilde{\rho} g, \\
\nabla \cdot \widetilde{\mathbf{v}}+\widetilde{w}_{z}=0, \\
\widetilde{T}_{t}+(\widetilde{\mathbf{v}} \cdot \nabla) \widetilde{T}+\widetilde{w} \widetilde{T}_{z}=Q_{\widetilde{T}}, \\
\widetilde{\rho}=\rho_{0}\left(1-\alpha\left(\widetilde{T}-T_{0}\right)\right) .
\end{array}\right.
$$


Here $\widetilde{\mathbf{v}}=(\widetilde{u}, \widetilde{v})$ is the horizontal velocity, $\widetilde{w}$ the vertical velocity; $\widetilde{\rho}$ is the density, $\widetilde{p}$ the pressure, and $\widetilde{T}$ the temperature; $\nabla$ denotes the horizontal gradient operator; $\widetilde{\mathbf{v}}_{t}=\partial \mathbf{v} / \partial t$, etc. The independent variables are $(x, y, z) \in \mathcal{M}=\left(0, L_{1}\right) \times\left(0, L_{2}\right) \times\left(-L_{3}, 0\right)$, and $t>0$.

As said before, in the physical context the forcing terms $F_{\widetilde{\mathbf{v}}}=$ $\left(F_{\widetilde{u}}, F_{\widetilde{v}}\right)$ and $Q_{\widetilde{T}}$ vanish, but we introduce them here for mathematical generality and, below, to study the case of nonhomogeneous boundary conditions by homogenization of the boundary conditions.

After introducing the basic stratified flow, following the steps of Section 1 , we reach the following system with five equations and five unknowns:

$$
\left\{\begin{array}{l}
u_{t}+\bar{U}_{0} u_{x}-f v+\phi_{x}=0, \\
v_{t}+\bar{U}_{0} v_{x}+f\left(\bar{U}_{0}+u\right)+\phi_{y}=0, \\
\psi_{t}+\bar{U}_{0} \psi_{x}+N^{2} w=0 \\
u_{x}+v_{y}+w_{z}=0 \\
\phi_{z}=\psi
\end{array}\right.
$$

The functions $u, v, \ldots$, are related to $\widetilde{u}, \widetilde{v}$ by $(1.7)$.

3.2.1. Finite Element Expansion in the $y$-Direction. The aim is to find (and numerically study) a 2D version of (3.3), which is physically interesting. For that purpose we want the flow to be close to geostrophic equilibrium, so that $u=u^{g}+u^{\prime}$, or $\widetilde{u}=\bar{u}+u^{g}+u^{\prime}{ }^{11}$, etc., where $u^{g}$, etc. (and as well $\bar{u}+u^{g}$, etc.), are geostrophic, and $u^{\prime}, v^{\prime}$, etc. are small compared to $u^{g}, v^{g}$, etc., which are themselves small compared to $\bar{u}, \bar{v}$, etc.

The geostrophic equation

$$
p_{y}^{g}=-\rho_{0} f u^{g}
$$

prevents us from taking functions $\widetilde{u}=u^{g}+u, \widetilde{p}=p^{g}+p, \ldots$, independent of $y$. Indeed if we consider a space periodic approximation with two or three modes of the Fourier series in $y$, (3.4) will introduce the Fourier series expansion of

$$
h(y)=y, \quad 0<y<L_{2},
$$

and, as is well-known, the discontinuity of (the periodic extension of) $h$ leads to numerical oscillations.

\footnotetext{
${ }^{11}$ The notations $u^{\prime}, v^{\prime}$, etc. are not used in the sequel.
} 

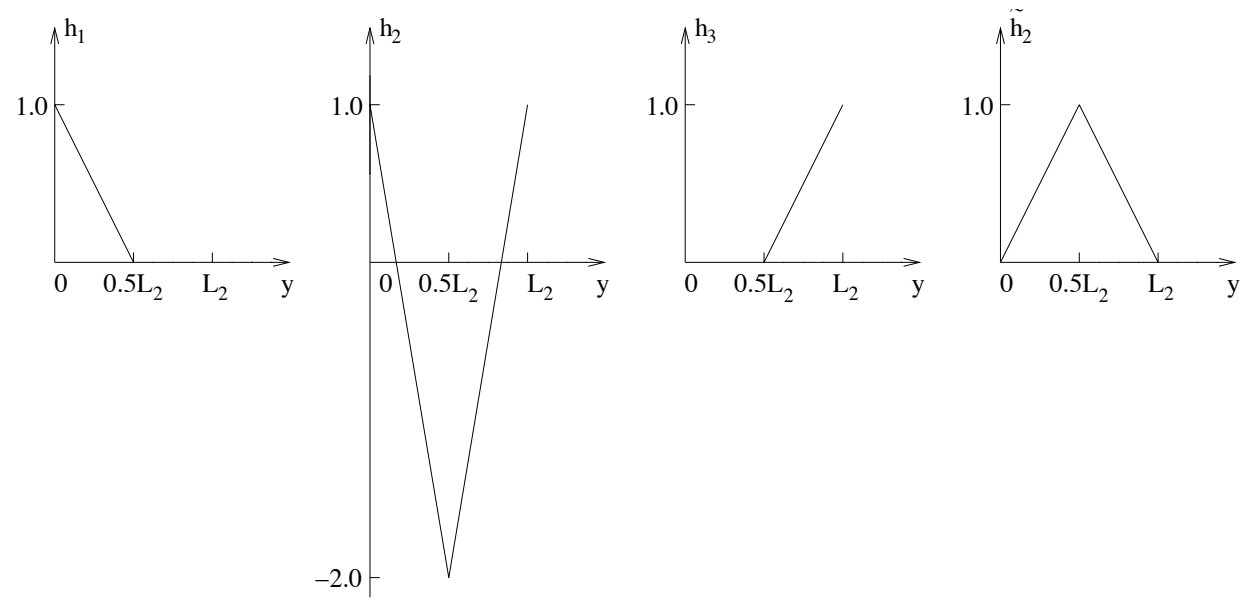

Figure 17 . The hat functions $h_{1}, h_{2}, h_{3}$ and $\widetilde{h}_{2}$.

Hence our 2.5D model will allow linear variations in $y$, and, in view of (3.4) it is then natural to introduce piecewise linear finite elements in the $y$ direction. We introduce one middle point $0.5 L_{2}$ in the middle of the interval $\left(0, L_{2}\right): 0, L_{2}$ play the role of boundaries, and values at $0.5 L_{2}$ play the role of the flow "independent of $y$ ". We introduce three hat functions (finite elements) $h_{1}, h_{2}$, and $h_{3}$ (see Figure 3.2.1) corresponding to the points $0,0.5 L_{2}$, and $L_{2}$. Instead of the usual hat function $\widetilde{h}_{2}$ (see Figure 3.2.1), we use $h_{2}$ such that $h_{1}, h_{2}$, and $h_{3}$ are orthogonal.

We now look for approximate solutions of (3.3) of the form of

$$
\left\{\begin{array}{l}
u \simeq u_{1}(x, z, t) h_{1}(y)+u_{2}(x, z, t) h_{2}(y)+u_{3}(x, z, t) h_{3}(y) \\
v \simeq v_{1}(x, z, t) h_{1}(y)+v_{2}(x, z, t) h_{2}(y)+v_{3}(x, z, t) h_{3}(y) \\
w \simeq w_{1}(x, z, t) h_{1}(y)+w_{2}(x, z, t) h_{2}(y)+w_{3}(x, z, t) h_{3}(y) \\
\phi \simeq \phi_{1}(x, z, t) h_{1}(y)+\phi_{2}(x, z, t) h_{2}(y)+\phi_{3}(x, z, t) h_{3}(y) \\
\psi \simeq \psi_{1}(x, z, t) h_{1}(y)+\psi_{2}(x, z, t) h_{2}(y)+\psi_{3}(x, z, t) h_{3}(y)
\end{array}\right.
$$

and consider the corresponding finite elements (Galerkin) approximation of (3.3). We then introduce the expressions (3.6) for $u, v, w, \phi$ and $\psi$ into the system (3.3), multiply each equation by $h_{1}, h_{2}$ and $h_{3}$ respectively, and integrate over $(0,1)$. Thanks to the orthogonality of 
$h_{1}, h_{2}$ and $h_{3}$, we obtain the following system:

$$
\left\{\begin{array}{l}
\boldsymbol{u}_{t}+\bar{U}_{0} \boldsymbol{u}_{x}+\phi_{x}-f \boldsymbol{v}=0, \\
\boldsymbol{v}_{t}+\bar{U}_{0} \boldsymbol{v}_{x}+f \boldsymbol{u}+\Lambda \boldsymbol{\phi}+\boldsymbol{f}=0, \\
\boldsymbol{\psi}_{t}+\bar{U}_{0} \psi_{x}+N^{2} \boldsymbol{w}=0 \\
\boldsymbol{u}_{x}+\Lambda \boldsymbol{v}+\boldsymbol{w}_{z}=0 \\
\boldsymbol{\psi}=\boldsymbol{\phi}_{z}
\end{array}\right.
$$

Here

$$
\begin{aligned}
\boldsymbol{u} & =\left(u_{1}, u_{2}, u_{3}\right)^{T}, \quad \boldsymbol{v}=\left(v_{1}, v_{2}, v_{3}\right)^{T}, \quad \boldsymbol{\phi}=\left(\phi_{1}, \phi_{2}, \phi_{3}\right)^{T} \\
\boldsymbol{\psi} & =\left(\psi_{1}, \psi_{2}, \psi_{3}\right)^{T}, \quad \boldsymbol{w}=\left(w_{1}, w_{2}, w_{3}\right)^{T},
\end{aligned}
$$

and

$$
\Lambda=\frac{1}{L_{2}}\left(\begin{array}{ccc}
-3 & -9 & 0 \\
\frac{1}{2} & 0 & -\frac{1}{2} \\
0 & 9 & 3
\end{array}\right) \quad \boldsymbol{f}=\frac{1}{L_{2}}\left(\begin{array}{c}
\frac{3}{2} f \bar{U}_{0} \\
-\frac{1}{2} f \bar{U}_{0} \\
\frac{3}{2} f \bar{U}_{0}
\end{array}\right)
$$

Note that the matrix $\Lambda$ which has the physical dimension of $l e n g t h^{-1}$ can be basically seen as the discretized form, in the context of this Galerkin procedure, of the differential operator $\partial / \partial y$.

We denote by $\mathcal{M}^{\prime}=\left(0, L_{1}\right) \times\left(-L_{3}, 0\right)$ the two-dimensional spatial domain for the system (3.7).

3.2.2. The normal mode expansion. As in Section 2 for the $2 \mathrm{D}$ case, we consider a normal mode expansion of the solutions of system (3.7). That is, we look for solutions of this system in the form:

$$
\left\{\begin{array}{l}
(\boldsymbol{u}, \boldsymbol{v}, \boldsymbol{\phi})=\sum_{n \geq 0} \mathcal{U}_{n}(z)\left(\boldsymbol{u}_{n}, \boldsymbol{v}_{n}, \boldsymbol{\phi}_{n}\right)(x, t) \\
(\boldsymbol{w}, \boldsymbol{\psi})=\sum_{n \geq 1} \mathcal{W}_{n}(z)\left(\boldsymbol{w}_{n}, \boldsymbol{\psi}_{n}\right)(x, t)
\end{array}\right.
$$

Here $\boldsymbol{u}_{n}, \boldsymbol{v}_{n}$, etc., are vector functions as $\boldsymbol{u}, \boldsymbol{v}$, etc. , but are independent of $z$. We refer the reader to Section 1 (or Temam and Tribbia (2003)) for the justification of the normal mode expansion. The specifications of the eigenfunctions $\mathcal{U}_{n}$ and $\mathcal{W}_{n}$ given in Section 2 are repeated here for convenience of the reader:

$$
\left\{\begin{array}{l}
\mathcal{U}_{0}=\sqrt{\frac{1}{L_{3}}}, \text { and } \mathcal{U}_{n}=\sqrt{\frac{2}{L_{3}}} \cos \left(\lambda_{n} z\right) \quad \text { for } n \geq 1, \\
\mathcal{W}_{n}=\sqrt{\frac{2}{L_{3}}} \sin \left(\lambda_{n} z\right) \quad \text { for } n \geq 1,
\end{array}\right.
$$


where $\lambda_{n}=n \pi / L_{3}$. We observe that, for $n, m \geq 1$,

$$
\left\{\begin{array}{l}
\int_{-L_{3}}^{0} \mathcal{U}_{n}(z) \mathcal{U}_{m}(z) \mathrm{d} z=\delta_{n, m} \\
\int_{-L_{3}}^{0} \mathcal{W}_{n}(z) \mathcal{W}_{m}(z) \mathrm{d} z=\delta_{n, m} \\
\int_{-L_{3}}^{0} \mathcal{U}_{n}(z) \mathcal{W}_{m}(z) \mathrm{d} z=0 \\
\mathcal{U}_{n}^{\prime}(z)=-\lambda_{n} \mathcal{W}_{n}(z) \\
\mathcal{W}_{n}^{\prime}(z)=\lambda_{n} \mathcal{U}_{n}(z)
\end{array}\right.
$$

We then introduce (3.9) into the system (3.7). For each $n \geq 0$, we multiply each equation by $\mathcal{U}_{n}$ (or $\mathcal{W}_{n}$ for the 3 rd and 5 th equations), and integrate over $\left(-L_{3}, 0\right)$. When $n=0$, we obtain a system for $\boldsymbol{u}_{0}$, $\boldsymbol{v}_{0}$ and $\boldsymbol{\phi}_{0}$ only:

$$
\left\{\begin{array}{l}
\boldsymbol{u}_{0 t}+\bar{U}_{0} \boldsymbol{u}_{0 x}+\phi_{0 x}-f \boldsymbol{v}_{0}=0 \\
\boldsymbol{v}_{0 t}+\bar{U}_{0} \boldsymbol{v}_{0 x}+f \boldsymbol{u}_{0}+\Lambda \phi_{0}+\boldsymbol{f}_{0}=0 \\
\boldsymbol{u}_{0 x}+\Lambda \boldsymbol{v}_{0}=0
\end{array}\right.
$$

Here $\Lambda$ is the same as in (3.8), and

$$
\boldsymbol{f}_{0}=\sqrt{L_{3}} \boldsymbol{f}
$$

When $n \geq 1$, the corresponding system for each mode has the same form:

$$
\left\{\begin{array}{l}
\boldsymbol{u}_{n t}+\bar{U}_{0} \boldsymbol{u}_{n x}+\boldsymbol{\phi}_{n x}-f \boldsymbol{v}_{n}=0 \\
\boldsymbol{v}_{n t}+\bar{U}_{0} \boldsymbol{v}_{n x}+f \boldsymbol{u}_{n}+\Lambda \boldsymbol{\phi}_{n}=0 \\
\boldsymbol{\psi}_{n t}+\bar{U}_{0} \boldsymbol{\psi}_{n x}+N^{2} \boldsymbol{w}_{n}=0 \\
\boldsymbol{u}_{n x}+\Lambda \boldsymbol{v}_{n}+\lambda_{n} \boldsymbol{w}_{n}=0 \\
-\lambda_{n} \boldsymbol{\phi}_{n}=\boldsymbol{\psi}_{n}
\end{array}\right.
$$

From the last two equations we notice that

$$
\boldsymbol{\phi}_{n}=-\frac{1}{\lambda_{n}} \boldsymbol{\psi}_{n}, \quad \boldsymbol{w}_{n}=-\frac{1}{\lambda_{n}}\left(\boldsymbol{u}_{n x}+\Lambda \boldsymbol{v}_{n}\right)
$$

which means that $\boldsymbol{\phi}_{n}$ and $\boldsymbol{w}_{n}$ are determined by the other three unknowns, they are diagnostic variables. Then we can eliminate $\boldsymbol{\phi}_{n}$ and 
$\boldsymbol{w}_{n}$ in (3.14), and obtain a system for $\boldsymbol{u}_{n}, \boldsymbol{v}_{n}$ and $\boldsymbol{\psi}_{n}$, for each $n \geq 1$ :

$$
\left\{\begin{array}{l}
\boldsymbol{u}_{n t}+\bar{U}_{0} \boldsymbol{u}_{n x}-\frac{1}{\lambda_{n}} \boldsymbol{\psi}_{n x}-f \boldsymbol{v}_{n}=0 \\
\boldsymbol{v}_{n t}+\bar{U}_{0} \boldsymbol{v}_{n x}+f \boldsymbol{u}_{n}-\frac{1}{\lambda_{n}} \Lambda \boldsymbol{\psi}_{n}=0 \\
\boldsymbol{\psi}_{n t}-\frac{N^{2}}{\lambda_{n}} \boldsymbol{u}_{n x}+\bar{U}_{0} \boldsymbol{\psi}_{n x}-\frac{N^{2}}{\lambda_{n}} \Lambda \boldsymbol{v}_{n}=0
\end{array}\right.
$$

In Section 3.2.3 we will present the boundary conditions at $x=0$ and $L_{1}$ for the modes $n \geq 1$. These boundary conditions will ensure the well-posedness of the system (3.7), which we are going to establish in Section 3.3. Due to its different, and somehow irregular form, the system (3.12) of the zero mode will be treated separately at the end of Section 3.3.

3.2.3. Boundary Conditions at $x=0, L_{1}$. The analysis by which we determine the boundary conditions for the systems (3.16), and ultimately for (3.7), is similar to that in the 2D case. We will review the spirit of the analysis here for the sake of completeness, and then list the boundary conditions that we propose for the systems (3.16).

The matrix associated with the coefficients of the first order terms with respect to $x$ reads:

$$
\left(\begin{array}{ccc}
\bar{U}_{0} & 0 & -\frac{1}{\lambda_{n}} \\
0 & \bar{U}_{0} & 0 \\
-\frac{N^{2}}{\lambda_{n}} & 0 & \bar{U}_{0}
\end{array}\right) .
$$

There are three eigenvalues to this matrix, namely $\bar{U}_{0}+N / \lambda_{n}, \bar{U}_{0}$ and $\bar{U}_{0}-N / \lambda_{n}$. Because $\bar{U}_{0}$ and $\lambda_{n}$ are positive, each mode has at least two positive eigenvalues. However, as before and depending on $n$, the third eigenvalue $\bar{U}_{0}-1 / \lambda_{n}$ can be either positive or negative for the actual (physical) values of $\bar{U}_{0}$. We say that the corresponding mode is supercritical in the first case, and subcritical in the second case. The supercritical modes require three boundary conditions at $x=0$, while the subcritical modes require two boundary conditions at $x=0$ and one at $x=L_{1}$. This mandates that we impose different boundary conditions according to the type of the modes.

We first note that the sequence $\left\{\lambda_{n}\right\}$ is monotone and $\lambda_{n} \longrightarrow \infty$ as $n \longrightarrow \infty$. Therefore there are only a finite number of subcritical modes, which however are the most challenging and also the most important ones as they carry much energy. 
We continue to denote by $n_{c}$ the number of subcritical modes, which is defined as in (1.23):

$$
\begin{gathered}
\lambda_{n_{c}}=\frac{n_{c} \pi}{L_{3}}<\frac{1}{\bar{U}_{0}}, \\
\lambda_{n_{c}+1}=\frac{\left(n_{c}+1\right) \pi}{L_{3}}>\frac{1}{\bar{U}_{0}} .
\end{gathered}
$$

Remark 3.1. When $\bar{U}_{0}-N / \lambda_{n}=0$ for some $n$, the mode will be neither subcritical, nor supercritical. But this can be easily avoided by modifying as necessary the velocity of the reference flow. For this reason we assume as in (1.24) that $\bar{U}_{0} \neq N / \lambda_{n}$ for all $n$ 's.

For the supercritical modes, i.e. when $n>n_{c}$, we take the natural boundary conditions:

$$
\left\{\begin{array}{l}
\boldsymbol{u}_{n}(0, t)=0 \\
\boldsymbol{v}_{n}(0, t)=0 \\
\boldsymbol{\psi}_{n}(0, t)=0
\end{array}\right.
$$

For the subcritical modes, i.e. when $1 \leq n \leq n_{c}$, we impose the boundary conditions in the following way:

$$
\left\{\begin{array}{l}
\boldsymbol{\xi}_{n}(0, t)=0 \\
\boldsymbol{v}_{n}(0, t)=0 \\
\boldsymbol{\eta}_{n}\left(L_{1}, t\right)=0
\end{array}\right.
$$

Here $\boldsymbol{\xi}_{n}=\boldsymbol{u}_{n}-\boldsymbol{\psi}_{n} / N, \boldsymbol{v}_{n}=\boldsymbol{v}_{n}$, and $\boldsymbol{\eta}_{n}=\boldsymbol{u}_{n}+\boldsymbol{\psi}_{n} / N$ are the three eigenvectors corresponding to $\bar{U}_{0}+N / \lambda_{n}, \bar{U}_{0}$ and $\bar{U}_{0}-N / \lambda_{n}$ respectively.

Remark 3.2. In this subsection the boundary conditions are given for each mode. The boundary conditions for the system (3.7) will come directly from (3.17) and (3.18), and will be presented later on (see (3.22) and (3.23)).

Remark 3.3. For most of this section the boundary conditions will be homogeneous. But at the end we will explain how to handle the nonhomogeneous case. Some technicalities related to the so-called compatibility conditions will appear.

\subsection{Well-Posedness of the Linear System.}


3.3.1. The functional setting. We want to write (3.7) (the zero mode excluded, see Remark 3.4 below) as an initial value problem of the form

$$
\left\{\begin{array}{l}
\frac{\mathrm{d} U}{\mathrm{~d} t}+A U=F, \\
U(0)=U_{0} .
\end{array}\right.
$$

Here $U=U(t)$ stands for $(\boldsymbol{u}(t), \boldsymbol{v}(t), \boldsymbol{\psi}(t))$, and $A$ is an unbounded operator in $H$ with domain $D(A) \subset H$, and $U_{0} \in D(A), F \in H$. The space $H$ is defined as follows:

$$
H=H_{\boldsymbol{u}} \times H_{\boldsymbol{v}} \times H_{\boldsymbol{\psi}}
$$

where

$$
\begin{aligned}
& H_{\boldsymbol{u}}=H_{\boldsymbol{v}}=\left\{\boldsymbol{u} \in \mathbf{L}^{2}\left(\mathcal{M}^{\prime}\right) \mid \int_{-L_{3}}^{0} \boldsymbol{u}(x, z) \mathrm{d} z=\mathbf{0}, \text { for a.e. } x \in\left(0, L_{1}\right)\right\}, \\
& H_{\boldsymbol{\psi}}=\mathbf{L}^{2}\left(\mathcal{M}^{\prime}\right) .
\end{aligned}
$$

In the definitions above $\mathcal{M}^{\prime}$ is the two dimensional domain $\left(0, L_{1}\right) \times$ $\left(-L_{3}, 0\right)$ (see at the end of Section 3.2.1). The convention that $\mathbf{L}^{2}\left(\mathcal{M}^{\prime}\right)=$ $\left(L^{2}\left(\mathcal{M}^{\prime}\right)\right)^{3}$ has been used. Similarly, later in this section, we will use $\mathbf{H}^{1}\left(\mathcal{M}^{\prime}\right), \mathcal{D}\left(\mathcal{M}^{\prime}\right)$, etc., for the corresponding vector function spaces; $H$ is endowed with the following scalar product:

$$
(U, \widetilde{U})_{H}=\int_{\mathcal{M}^{\prime}}\left(\boldsymbol{u} \cdot \widetilde{\boldsymbol{u}}+\boldsymbol{v} \cdot \widetilde{\boldsymbol{v}}+\frac{1}{N^{2}} \boldsymbol{\psi} \cdot \widetilde{\boldsymbol{\psi}}\right) \mathrm{d} \mathcal{M}^{\prime} .
$$

Clearly $H$ is a closed subspace of $\left(\mathbf{L}^{2}\left(\mathcal{M}^{\prime}\right)\right)^{3}$, and the norm of $H$ derived from the scalar product $(\cdot, \cdot)_{H}$ is equivalent to that of $\left(\mathbf{L}^{2}\left(\mathcal{M}^{\prime}\right)\right)^{3}$.

We denote by $P$ the orthogonal projector from $\mathbf{L}^{2}\left(\mathcal{M}^{\prime}\right)$ onto $H_{\boldsymbol{u}}$ (and also onto $H_{\boldsymbol{v}}$, since $H_{\boldsymbol{u}}$ and $H_{\boldsymbol{v}}$ are identical.) Hence, for each $\boldsymbol{g} \in \mathbf{L}^{2}\left(\mathcal{M}^{\prime}\right)$

$$
\left\{\begin{array}{l}
P(\boldsymbol{g})=\boldsymbol{g}-\frac{1}{L_{3}} \int_{-L_{3}}^{0} \underset{\boldsymbol{g}}{\boldsymbol{g}}(x, z) \mathrm{d} z \\
(I-P)(\boldsymbol{g})=\frac{1}{L_{3}} \int_{-L_{3}}^{0} \boldsymbol{g}(x, z) \mathrm{d} z
\end{array}\right.
$$

We can easily check that $P(\boldsymbol{g}) \in H_{\boldsymbol{u}}$, and $(I-P)(\boldsymbol{g}) \in H_{\boldsymbol{u}}^{\perp}$. We can also show that $H_{\boldsymbol{u}}^{\perp}=\mathbf{L}_{x}^{2}\left(0, L_{1}\right)$. Indeed, for each $\boldsymbol{f} \in \mathbf{L}_{x}^{2}\left(0, L_{1}\right), P(\boldsymbol{f})=\mathbf{0}$, and so $\boldsymbol{f} \in H_{\boldsymbol{u}}^{\perp}$. If, on the other hand, $\boldsymbol{f} \in H_{\boldsymbol{u}}^{\perp}$, then $(I-P) \boldsymbol{f}=\boldsymbol{f}$. Hence $\boldsymbol{f}$ is independent of $z$, and $\boldsymbol{f} \in \mathbf{L}_{x}^{2}\left(0, L_{1}\right)$.

The unknown $U$ is subjected to modal boundary conditions, which are listed below. The parallelism between the modal boundary conditions for $U$ and the boundary conditions for each mode (see (3.17) and 
(3.18)) is obvious.

For $n>n_{c}$ (i.e. for the the supercritical modes),

$$
\left\{\begin{array}{l}
\int_{-L_{3}}^{0} \boldsymbol{u}(0, z) \mathcal{U}_{n}(z) \mathrm{d} z=\mathbf{0} \\
\int_{-L_{3}}^{0} \boldsymbol{v}(0, z) \mathcal{U}_{n}(z) \mathrm{d} z=\mathbf{0} \\
\int_{-L_{3}}^{0} \boldsymbol{\psi}(0, z) \mathcal{W}_{n}(z) \mathrm{d} z=\mathbf{0}
\end{array}\right.
$$

For $1 \leq n \leq n_{c}$ (i.e. for the subcritical modes),

$$
\left\{\begin{array}{l}
\int_{-L_{3}}^{0} \boldsymbol{u}(0, z) \mathcal{U}_{n}(z)-\frac{1}{N} \int_{-L_{3}}^{0} \boldsymbol{\psi}(0, z) \mathcal{W}_{n}(z) \mathrm{d} z=\mathbf{0} \\
\int_{-L_{3}}^{0} \boldsymbol{v}(0, z) \mathcal{U}_{n}(z)=\mathbf{0} \\
\int_{-L_{3}}^{0} \boldsymbol{u}\left(L_{1}, z\right) \mathcal{U}_{n}(z)+\frac{1}{N} \int_{-L_{3}}^{0} \boldsymbol{\psi}\left(L_{1}, z\right) \mathcal{W}_{n}(z) \mathrm{d} z=\mathbf{0}
\end{array}\right.
$$

We now define $D(A)$ as follows:

$$
\begin{aligned}
D(A)=\{U \in H & \mid U_{x} \in\left(\mathbf{L}^{2}\left(\mathcal{M}^{\prime}\right)\right)^{3} \\
& \text { and Uverifies the BC's (3.22) and (3.23) }\} .
\end{aligned}
$$

Then for every $U \in D(A), A U$ is defined by

$$
A U=\left(\begin{array}{c}
\bar{U}_{0} \boldsymbol{u}_{x}-f \boldsymbol{v}-P\left[\int_{z}^{0} \boldsymbol{\psi}_{x}\left(x, z^{\prime}\right) \mathrm{d} z^{\prime}\right] \\
\bar{U}_{0} \boldsymbol{v}_{x}+f \boldsymbol{u}-P\left[\int_{z}^{\overline{0}} \Lambda \boldsymbol{\psi}\left(x, z^{\prime}\right) \mathrm{d} z^{\prime}\right] \\
\bar{U}_{0} \boldsymbol{\psi}_{x}+N^{2} \int_{z}^{0}\left(\boldsymbol{u}_{x}+\Lambda \boldsymbol{v}\right) \mathrm{d} z^{\prime}
\end{array}\right) .
$$

Remark 3.4. By the definition of the spaces $H$ and $D(A)$, we include in the system (3.19) all the modes with $n \geq 1$. The zero mode $(n=0)$ is excluded from the system, and will be treated separately.

3.3.2. Main Result. We will prove the well-posedness of the system (3.19) with the help of the Hille-Yosida theorem, Theorem 2.1. The main result of this section concerning (3.19) is as follows;

Theorem 3.5. Let $H, A$ and $D(A)$ be defined as in Section 3.3.1. Then the initial value problem (3.19) is well-posed. That is, for every $t_{1}>0$, and for every $U_{0} \in D(A), F \in L^{1}\left(0, t_{1} ; H\right)$, with $F^{\prime} \in$ 
$L^{1}\left(0, t_{1} ; H\right)$, (3.19) has a unique solution $U$ such that

$$
U \in C\left(\left[0, t_{1}\right] ; H\right) \cap L^{\infty}\left(0, t_{1} ; D(A)\right), \quad \frac{\mathrm{d} U}{\mathrm{~d} t} \in L^{\infty}\left(0, t_{1} ; H\right) .
$$

3.3.3. Proof of Theorem 3.5. We first want to rewrite $A U$ in another form, and we also want to introduce the adjoint $A^{*}$ of $A$ and its domain $D\left(A^{*}\right)$, which are needed in the course of the proof.

We want to express $A U$ in terms of $\boldsymbol{u}_{n}, \boldsymbol{v}_{n}$ and $\boldsymbol{\psi}_{n}$. This form is more convenient for the calculations. To this end, we simply introduce the normal mode expansions (3.9) of $\boldsymbol{u}, \boldsymbol{v}$ and $\boldsymbol{\psi}$ into (3.25). Note that, since $\boldsymbol{u} \in H_{\boldsymbol{u}}$ and $\boldsymbol{v} \in H_{\boldsymbol{v}}, \boldsymbol{u}_{0}$ and $\boldsymbol{v}_{0}$ vanish. After working out the integrations, and grouping the coefficients of the eigenfunctions we obtain

$$
A U=\left(\begin{array}{c}
\sum_{n \geq 1}\left(\bar{U}_{0} \boldsymbol{u}_{n x}-\frac{1}{\lambda_{n}} \boldsymbol{\psi}_{n x}-f \boldsymbol{v}_{n}\right) \mathcal{U}_{n} \\
\sum_{n \geq 1}\left(\bar{U}_{0} \boldsymbol{v}_{n x}+f \boldsymbol{u}_{n}-\frac{1}{\lambda_{n}} \Lambda \boldsymbol{\psi}_{n}\right) \mathcal{U}_{n} \\
\sum_{n \geq 1}\left(-\frac{N^{2}}{\lambda_{n}} \boldsymbol{u}_{n x}+\bar{U}_{0} \boldsymbol{\psi}_{n x}-\frac{N^{2}}{\lambda_{n}} \Lambda \boldsymbol{v}_{n}\right) \mathcal{W}_{n}
\end{array}\right)
$$

We recall (see e.g. Rudin (1991)) that, given an unbounded operator $A$ from $D(A)$ into $H$, the domain of its adjoint consists in the $U$ in $H$ such that $U \longrightarrow(A U, \widetilde{U})$ is a linear functional $K$ on $D(A)$, continuous for the norm of $H$, in which case $A^{*} \widetilde{U}=K$. The determination of $A^{*}$ introduces the following boundary conditions for $\widetilde{U}$ :

For the the supercritical modes, i.e. $n>n_{c}$,

$$
\left\{\begin{array}{l}
\widetilde{\boldsymbol{u}}_{n}(0, t)=0, \\
\widetilde{\boldsymbol{v}}_{n}(0, t)=0, \\
\widetilde{\boldsymbol{\psi}}_{n}(0, t)=0 .
\end{array}\right.
$$

For the the subcritical modes, i.e. $1 \leq n \leq n_{c}$,

$$
\left\{\begin{array}{l}
\widetilde{\boldsymbol{u}}_{n}\left(L_{1}\right)-\frac{1}{N} \widetilde{\boldsymbol{\psi}}_{n}\left(L_{1}\right)=0, \\
\widetilde{\boldsymbol{v}}_{n}\left(L_{1}\right)=0 \\
\widetilde{\boldsymbol{u}}_{n}(0)+\frac{1}{N} \widetilde{\boldsymbol{\psi}}_{n}(0)=0 .
\end{array}\right.
$$


A simple analysis, which we skip ${ }^{12}$, shows that the domain $D\left(A^{*}\right)$ of $A^{*}$ is as follows:

$$
\begin{aligned}
& D\left(A^{*}\right)=\left\{\widetilde{U} \in H \mid \widetilde{U}_{x} \in\left(\mathbf{L}^{2}\left(\mathcal{M}^{\prime}\right)^{3}\right.\right. \\
& \text { and } \widetilde{U} \text { verifies the BC's (3.28) and (3.29) }\} .
\end{aligned}
$$

Furthermore for $\widetilde{U} \in D\left(A^{*}\right)$,

$$
A^{*} \widetilde{U}=\left(\begin{array}{c}
\sum_{n \geq 1}\left(-\bar{U}_{0} \widetilde{\boldsymbol{u}}_{n x}+\frac{1}{\lambda_{n}} \widetilde{\boldsymbol{\psi}}_{n x}+f \widetilde{\boldsymbol{v}}_{n}\right) \mathcal{U}_{n} \\
\sum_{n \geq 1}\left(-\bar{U}_{0} \widetilde{\boldsymbol{v}}_{n x}-f \widetilde{\boldsymbol{u}}_{n}-\frac{1}{\lambda_{n}} \Lambda^{T} \widetilde{\boldsymbol{\psi}}_{n}\right) \mathcal{U}_{n} \\
\sum_{n \geq 1}\left(\frac{N^{2}}{\lambda_{n}} \widetilde{\boldsymbol{u}}_{n x}-\bar{U}_{0} \widetilde{\boldsymbol{\psi}}_{n x}-\frac{N^{2}}{\lambda_{n}} \Lambda^{T} \widetilde{\boldsymbol{v}}_{n}\right) \mathcal{W}_{n}
\end{array}\right) .
$$

The coefficient matrix $\Lambda$ in (3.31) is the same as in (3.8), and $\Lambda^{T}$ is its transpose.

The proof of Theorem 3.5 essentially consists of the verification of the hypotheses of Theorem 2.1. We will do it in the Lemmas 3.1 to 3.5. We will then summarize the proof of Theorem 3.5 at the end of this section.

Lemma 3.1. There exists $\delta>0$ such that $A+\delta I \geq 0$ and $A^{*}+$ $\delta I \geq 0$, that is, $((A+\delta I) U, U)_{H} \geq 0$ for each $U \in D(A)$ and $\left(\left(A^{*}+\right.\right.$ $\delta I) \widetilde{\widetilde{U}}, \widetilde{U})_{H} \geq 0$ for each $\widetilde{U} \in D\left(A^{*}\right)$.

Proof. Let $U \in D(A)$. Then $U$ has the normal mode expansion

$$
U=\left(\sum_{n \geq 1} \boldsymbol{u}_{n} \mathcal{U}_{n}, \sum_{n \geq 1} \boldsymbol{v}_{n} \mathcal{U}_{n}, \sum_{n \geq 1} \boldsymbol{\psi}_{n} \mathcal{W}_{n}\right)
$$

\footnotetext{
${ }^{12}$ See in Section 4 a more involved analysis in dimension 3.
} 
Using the expression (3.27) for $A U$, we compute,

$$
\begin{aligned}
(A U, U)_{H}= & \int_{0}^{L_{1}} \int_{-L_{3}}^{0} A U \cdot U \mathrm{~d} z \mathrm{~d} x \\
= & \int_{0}^{L_{1}}\left[\sum_{n \geq 1}\left(\bar{U}_{0} \boldsymbol{u}_{n x} \cdot \boldsymbol{u}_{n}-\frac{1}{\lambda_{n}} \boldsymbol{\psi}_{n x} \cdot \boldsymbol{u}_{n}-f \boldsymbol{v}_{n} \cdot \boldsymbol{u}_{n}\right)\right. \\
& +\sum_{n \geq 1}\left(\bar{U}_{0} \boldsymbol{v}_{n x} \cdot \boldsymbol{v}_{n}+f \boldsymbol{u}_{n} \cdot \boldsymbol{v}_{n}-\frac{1}{\lambda_{n}} \Lambda \boldsymbol{\psi}_{n} \cdot \boldsymbol{v}_{n}\right) \\
& \left.+\sum_{n \geq 1}\left(-\frac{1}{\lambda_{n}} \boldsymbol{u}_{n x} \cdot \boldsymbol{\psi}_{n}+\frac{\bar{U}_{0}}{N^{2}} \boldsymbol{\psi}_{n x} \cdot \boldsymbol{\psi}_{n}-\frac{1}{\lambda_{n}} \Lambda \boldsymbol{v}_{n} \cdot \boldsymbol{\psi}_{n}\right)\right] \mathrm{d} x \\
= & \sum_{n \geq 1}\left[\frac{\bar{U}_{0}}{2} \boldsymbol{u}_{n}^{2}\left(L_{1}\right)-\frac{\bar{U}_{0}}{2} \boldsymbol{u}_{n}{ }^{2}(0)+\frac{\bar{U}_{0}}{2} \boldsymbol{v}_{n}{ }^{2}\left(L_{1}\right)-\frac{\bar{U}_{0}}{2} \boldsymbol{v}_{n}{ }^{2}(0)\right. \\
& +\frac{\bar{U}_{0}}{2 N^{2}} \boldsymbol{\psi}_{n}{ }^{2}\left(L_{1}\right)-\frac{\bar{U}_{0}}{2 N^{2}} \boldsymbol{\psi}_{n}{ }^{2}(0) \\
& -\frac{1}{\lambda_{n}} \boldsymbol{u}_{n}\left(L_{1}\right) \cdot \boldsymbol{\psi}_{n}\left(L_{1}\right)+\frac{1}{\lambda_{n}} \boldsymbol{u}_{n}(0) \cdot \boldsymbol{\psi}_{n}(0) \\
& \left.-\frac{1}{\lambda_{n}} \int_{0}^{L_{1}}\left(\Lambda \boldsymbol{\psi}_{n} \cdot \boldsymbol{v}_{n}+\Lambda \boldsymbol{v}_{n} \cdot \boldsymbol{\psi}_{n}\right) \mathrm{d} x\right]
\end{aligned}
$$

We now separate the supercritical and subcritical modes, and drop those terms that vanish according to the boundary conditions (3.17), (3.18). There remains:

$$
\begin{aligned}
(A U, U)= & \sum_{n>n_{c}}\left[\frac{\bar{U}_{0}}{2} \boldsymbol{u}_{n}^{2}\left(L_{1}\right)+\frac{\bar{U}_{0}}{2} \boldsymbol{v}_{n}^{2}\left(L_{1}\right)+\frac{\bar{U}_{0}}{2 N^{2}} \boldsymbol{\psi}_{n}^{2}\left(L_{1}\right)\right. \\
& \left.-\frac{1}{\lambda_{n}} \boldsymbol{u}_{n}\left(L_{1}\right) \cdot \boldsymbol{\psi}_{n}\left(L_{1}\right)\right]+ \\
& \sum_{1 \leq n \leq n_{c}}\left[\frac{\bar{U}_{0}}{2} \boldsymbol{u}_{n}^{2}\left(L_{1}\right)-\frac{\bar{U}_{0}}{2} \boldsymbol{u}_{n}^{2}(0)+\frac{\bar{U}_{0}}{2} \boldsymbol{v}_{n}{ }^{2}\left(L_{1}\right)\right. \\
& +\frac{\bar{U}_{0}}{2 N^{2}} \boldsymbol{\psi}_{n}^{2}\left(L_{1}\right)-\frac{\bar{U}_{0}}{2 N^{2}} \boldsymbol{\psi}_{n}^{2}(0) \\
& \left.-\frac{1}{\lambda_{n}} \boldsymbol{u}_{n}\left(L_{1}\right) \cdot \boldsymbol{\psi}_{n}\left(L_{1}\right)+\frac{1}{\lambda_{n}} \boldsymbol{u}_{n}(0) \cdot \boldsymbol{\psi}_{n}(0)\right]+ \\
& \sum_{n \geq 1} \frac{1}{\lambda_{n}} \int_{0}^{L_{1}}\left(-\Lambda \boldsymbol{\psi}_{n} \cdot \boldsymbol{v}_{n}-\Lambda \boldsymbol{v}_{n} \cdot \boldsymbol{\psi}_{n}\right) \mathrm{d} x .
\end{aligned}
$$


We then write

$$
(A U, U)=\sum_{n>n_{c}} \mathrm{I}_{n}+\sum_{1 \leq n \leq n_{c}} \mathrm{II}_{n}+\mathrm{III}
$$

where

$$
\begin{aligned}
\mathrm{I}_{n}= & \frac{\bar{U}_{0}}{2} \boldsymbol{u}_{n}^{2}\left(L_{1}\right)+\frac{\bar{U}_{0}}{2} \boldsymbol{v}_{n}{ }^{2}\left(L_{1}\right)+\frac{\bar{U}_{0}}{2 N^{2}} \boldsymbol{\psi}_{n}{ }^{2}\left(L_{1}\right) \\
& -\frac{1}{\lambda_{n}} \boldsymbol{u}_{n}\left(L_{1}\right) \cdot \boldsymbol{\psi}_{n}\left(L_{1}\right), \\
\mathrm{II}_{n}= & \frac{\bar{U}_{0}}{2} \boldsymbol{u}_{n}^{2}\left(L_{1}\right)-\frac{\bar{U}_{0}}{2} \boldsymbol{u}_{n}{ }^{2}(0)+\frac{\bar{U}_{0}}{2} \boldsymbol{v}_{n}{ }^{2}\left(L_{1}\right)+\frac{\bar{U}_{0}}{2 N^{2}} \boldsymbol{\psi}_{n}{ }^{2}\left(L_{1}\right) \\
& -\frac{\bar{U}_{0}}{2 N^{2}} \boldsymbol{\psi}_{n}{ }^{2}(0)-\frac{1}{\lambda_{n}} \boldsymbol{u}_{n}\left(L_{1}\right) \cdot \boldsymbol{\psi}_{n}\left(L_{1}\right)+\frac{1}{\lambda_{n}} \boldsymbol{u}_{n}(0) \cdot \boldsymbol{\psi}_{n}(0), \\
\mathrm{III}= & \sum_{n \geq 1} \frac{1}{\lambda_{n}} \int_{0}^{L_{1}}\left(-\Lambda \boldsymbol{\psi}_{n} \cdot \boldsymbol{v}_{n}-\Lambda \boldsymbol{v}_{n} \cdot \boldsymbol{\psi}_{n}\right) \mathrm{d} x .
\end{aligned}
$$

We see that $\mathrm{I}_{n}$ is the sum of a quadratic form and the positive term $\bar{U}_{0} \boldsymbol{v}_{n}^{2}\left(L_{1}\right) / 2$. We find the determinant for the quadratic form to be $\lambda_{n}^{-2}-\bar{U}_{0}^{2} / N^{-2}$, which is $<0$, thanks to the fact that $\bar{U}_{0}-N / \lambda_{n}>0$ for each supercritical mode. Hence we have

$$
\mathrm{I}_{n} \geq 0, \quad \text { for each } n>n_{c} .
$$

Using the boundary conditions (3.18) for the subcritical modes we find that

$$
\begin{aligned}
\mathrm{II}_{n} & =\frac{\bar{U}_{0}}{2} \boldsymbol{v}_{n}^{2}\left(L_{1}\right)+\left(\bar{U}_{0}+\frac{N}{\lambda_{n}}\right) \boldsymbol{u}_{n}^{2}\left(L_{1}\right)+\left(\frac{N}{\lambda_{n}}-\bar{U}_{0}\right) \boldsymbol{u}_{n}^{2}(0) \\
& \geq 0, \quad \text { for each } 1 \leq n \leq n_{c} .
\end{aligned}
$$

The last inequality is due to the fact that $\bar{U}_{0}-1 / \lambda_{n}<0$ for each subcritical mode. We also find an upper bound on the absolute value of III:

$$
\begin{aligned}
|\mathrm{III}| & \leq \sum_{n \geq 1} \frac{1}{N \lambda_{n}} \int_{0}^{L_{1}}\left|\left(\Lambda \boldsymbol{\psi}_{n} \cdot \boldsymbol{v}_{n}+\Lambda \boldsymbol{v}_{n} \cdot \boldsymbol{\psi}_{n}\right)\right| \mathrm{d} x \\
& \leq \sum_{n \geq 1} \frac{c_{1}^{\prime}}{\lambda_{n}}\left(\int_{0}^{L_{1}}\left|\frac{\boldsymbol{\psi}_{n}}{N}\right|^{2} \mathrm{~d} x\right)^{\frac{1}{2}}\left(\int_{0}^{L_{1}}\left|\boldsymbol{v}_{n}\right|^{2} \mathrm{~d} x\right)^{\frac{1}{2}} \\
& \leq \frac{c_{1}^{\prime}}{2 \lambda_{1}} \sum_{n \geq 1}\left(\int_{0}^{L_{1}}\left|\frac{\boldsymbol{\psi}_{n}}{N}\right|^{2} \mathrm{~d} x+\int_{0}^{L_{1}}\left|\boldsymbol{v}_{n}\right|^{2} \mathrm{~d} x\right),
\end{aligned}
$$


where $c_{1}^{\prime}$ is the norm of the matrix $\Lambda$. Hence, $\delta \geq c_{1}^{\prime} / 2 \lambda_{n}$ for every $n \geq 1$. Then

$$
|\mathrm{III}| \leq \frac{c_{1}^{\prime}}{2 \lambda_{1}}|U|_{H}^{2}
$$

and, for any $\delta>c_{1}^{\prime} / 2 \lambda_{1}$ the operator $A+\delta I$ is positive.

That $A^{*}+\delta I \geq 0$ (possibly with a different $\delta$ ) can be shown in a similar way. And we can always choose a constant $\delta$ such that the operators $A+\delta I$ and $A^{*}+\delta I$ are both positive. This completes the proof of Lemma 3.1.

Lemma 3.2. $D(A)$ is dense in $H$, and $A$ is a closed operator.

Proof. To show that $D(A)$ is dense in $\mathrm{H}$, consider $U=(\boldsymbol{u}, \boldsymbol{v}, \boldsymbol{\psi}) \in H$. Since $\left(\mathcal{D}\left(\mathcal{M}^{\prime}\right)\right)^{3}$, the set of $\mathcal{C}^{\infty}$ functions with compact support in $\mathcal{M}^{\prime}$, is dense in $\mathbf{L}^{2}\left(\mathcal{M}^{\prime}\right), U$ can be approximated in $\mathbf{L}^{2}\left(\mathcal{M}^{\prime}\right)$ by elements of $\left(\mathcal{D}\left(\mathcal{M}^{\prime}\right)\right)^{3}$, say $\Phi^{j}=\left(\Phi_{\boldsymbol{u}}^{j}, \Phi_{\boldsymbol{v}}^{j}, \Phi_{\boldsymbol{\psi}}^{j}\right), 1 \leq j \leq \infty$. Since $P$ is continuous in $\left(\mathbf{L}^{2}\left(\mathcal{M}^{\prime}\right)\right)^{3},\left(P \Phi_{\boldsymbol{u}}^{j}, P \Phi_{\boldsymbol{v}}^{j}, \Phi_{\boldsymbol{\psi}}^{j}\right)$ also converge to $P U=U$ in $H$ as $j \longrightarrow \infty$. The function $\Phi^{j}$ are compactly supported in $\mathcal{M}^{\prime}$, and, by the definition of $P$, the functions $P \Phi^{j}$ are also compactly supported in $\mathcal{M}^{\prime}$. The necessary boundary conditions are satisfied and it is then clear that $\left(P \Phi_{\boldsymbol{u}}^{j}, P \Phi_{\boldsymbol{v}}^{j}, \Phi_{\boldsymbol{\psi}}^{j}\right)$ belong to $D(A)$.

To show that $A$ is closed, we need to show that for a sequence $\left\{U^{j}\right\}_{j=1}^{\infty}$ in $D(A)$, such that

$$
\begin{gathered}
U^{j} \longrightarrow U \quad \text { in } H, \\
A U^{j} \longrightarrow F \quad \text { in } H,
\end{gathered}
$$

with $U, F \in H$, then $U \in D(A)$ and $F=A U$. For each component of $U^{j}, U$ and $F,(3.32)$ and (3.33) mean

$$
\left\{\begin{array}{cc}
\boldsymbol{u}^{j} \longrightarrow \boldsymbol{u} & \text { in } H_{\boldsymbol{u}} \\
\boldsymbol{v}^{j} \longrightarrow \boldsymbol{v} & \text { in } H_{\boldsymbol{v}} \\
\boldsymbol{\psi}^{j} \longrightarrow \boldsymbol{\psi} & \text { in } H_{\boldsymbol{\psi}}
\end{array}\right.
$$

and

$$
\left\{\begin{array}{l}
\bar{U}_{0} \boldsymbol{u}_{x}^{j}-f \boldsymbol{v}^{j}-P\left[\int_{z}^{0} \boldsymbol{\psi}_{x}^{j}\left(x, z^{\prime}\right) \mathrm{d} z^{\prime}\right] \longrightarrow F_{\boldsymbol{u}} \quad \text { in } H_{\boldsymbol{u}} \\
\bar{U}_{0} \boldsymbol{v}_{x}^{j}+f \boldsymbol{u}^{j}-P\left[\int_{z}^{0} \Lambda \boldsymbol{\psi}^{j}\left(x, z^{\prime}\right) \mathrm{d} z^{\prime}\right] \longrightarrow F_{\boldsymbol{v}} \quad \text { in } H_{\boldsymbol{v}} \\
\bar{U}_{0} \boldsymbol{\psi}_{x}^{j}+N^{2} \int_{z}^{0}\left(\boldsymbol{u}_{x}^{j}+\Lambda \boldsymbol{v}^{j}\right) \mathrm{d} z^{\prime} \longrightarrow F_{\boldsymbol{\psi}} \quad \text { in } H_{\boldsymbol{\psi}} .
\end{array}\right.
$$


With regard to each mode, (3.34) implies that

$$
\left\{\begin{aligned}
\boldsymbol{u}_{n}^{j} \longrightarrow \boldsymbol{u}_{n} & \text { in } \mathbf{L}^{2}\left(0, L_{1}\right), \\
\boldsymbol{v}_{n}^{j} \longrightarrow \boldsymbol{v}_{n} & \text { in } \mathbf{L}^{2}\left(0, L_{1}\right), \\
\boldsymbol{\psi}_{n}^{j} \longrightarrow \boldsymbol{\psi}_{n} & \text { in } \mathbf{L}^{2}\left(0, L_{1}\right)
\end{aligned}\right.
$$

(3.34) also implies that $\sum_{n \geq 1}\left|\boldsymbol{u}_{n}^{j}\right|_{L^{2}\left(0, L_{1}\right)}^{2}, \sum_{n \geq 1}\left|\boldsymbol{v}_{n}^{j}\right|_{L^{2}\left(0, L_{1}\right)}^{2}$, and $\sum_{n \geq 1}\left|\boldsymbol{\psi}_{n}^{j}\right|_{L^{2}\left(0, L_{1}\right)}^{2}$ are uniformly bounded in $j$, by a bound of the $\left|U^{n}\right|_{H}^{2}$. Similarly, (3.35) implies that

$$
\begin{cases}\bar{U}_{0} \boldsymbol{u}_{n x}^{j}-\frac{1}{\lambda_{n}} \boldsymbol{\psi}_{n x}^{j}-f \boldsymbol{v}_{n}^{j} \equiv F_{\boldsymbol{u}, n}^{j} \longrightarrow F_{\boldsymbol{u}, n} & \text { in } \mathbf{L}^{2}\left(0, L_{1}\right), \\ \bar{U}_{0} \boldsymbol{v}_{n x}^{j}+f \boldsymbol{u}_{n}^{j}-\frac{1}{\lambda_{n}} \Lambda \boldsymbol{\psi}_{n}^{j} \equiv F_{\boldsymbol{v}, n}^{j} \longrightarrow F_{\boldsymbol{v}, n} & \text { in } \mathbf{L}^{2}\left(0, L_{1}\right), \\ -\frac{N^{2}}{\lambda_{n}} \boldsymbol{u}_{n x}^{j}+\bar{U}_{0} \boldsymbol{\psi}_{n x}^{j}-\frac{N^{2}}{\lambda_{n}} \Lambda \boldsymbol{v}_{n}^{j} \equiv F_{\boldsymbol{\psi}, n}^{j} \longrightarrow F_{\boldsymbol{\psi}, n} \quad \text { in } \mathbf{L}^{2}\left(0, L_{1}\right),\end{cases}
$$

and that $\sum_{n \geq 1}\left|F_{\boldsymbol{u}, n}^{j}\right|_{L^{2}\left(0, L_{1}\right)}^{2}, \sum_{n \geq 1}\left|F_{\boldsymbol{v}, n}^{j}\right|_{L^{2}\left(0, L_{1}\right)}^{2}$, and $\sum_{n \geq 1}\left|F_{\boldsymbol{\psi}, n}^{j}\right|_{L^{2}\left(0, L_{1}\right)}^{2}$ are uniformly bounded in $j$, by a bound of the $\left|A U^{n}\right|_{H}^{2}$. By the second convergence in (3.37) we have

$$
\boldsymbol{v}_{n x}^{j}=\frac{1}{\bar{U}_{0}}\left(-f \boldsymbol{u}_{n}^{j}+\frac{1}{\lambda_{n}} \Lambda \boldsymbol{\psi}_{n}^{j}+F_{\boldsymbol{v}, n}^{j}\right) .
$$

Each term on the right hand side of (3.38) converges in $\mathbf{L}^{2}\left(0, L_{1}\right)$, and therefore $\boldsymbol{v}_{n x}^{j}$ also converges in $\mathbf{L}^{2}\left(0, L_{1}\right)$. In addition, since on the right-hand side of $(3.38), \sum_{n \geq 1}\left|\boldsymbol{u}_{n}^{j}\right|_{L^{2}\left(0, L_{1}\right)}^{2}, \sum_{n \geq 1}\left|\boldsymbol{\psi}_{n}^{j}\right|_{L^{2}\left(0, L_{1}\right)}^{2}$, and $\sum_{n \geq 1}\left|F_{\boldsymbol{v}, n}^{j}\right|_{L^{2}\left(0, L_{1}\right)}^{2}$ are all uniformly bounded in $j, \sum_{n \geq 1}\left|\boldsymbol{v}_{n x}^{j}\right|_{L^{2}\left(0, L_{1}\right)}^{2}$ is also uniformly bounded in $j$. These two facts imply that $\boldsymbol{v}_{x}^{j}$ converges in $\mathbf{L}^{2}\left(\mathcal{M}^{\prime}\right)$. Combining this result with (3.34), we conclude that $\boldsymbol{v}_{x}$ belongs to $\mathbf{L}^{2}\left(\mathcal{M}^{\prime}\right)$, and

$$
\boldsymbol{v}_{x}^{j} \longrightarrow \boldsymbol{v}_{x} \quad \text { in } \mathbf{L}^{2}\left(\mathcal{M}^{\prime}\right)
$$

By the first and third convergences in (3.37) we obtain

$$
\begin{aligned}
& \boldsymbol{u}_{n x}=\frac{1}{\bar{U}_{0}^{2}-N^{2} / \lambda_{n}^{2}}\left[\left(f \bar{U}_{0}+\frac{N^{2}}{\lambda_{n}^{2}} \Lambda\right) \boldsymbol{v}_{n}+\bar{U}_{0} F_{\boldsymbol{u}, n}+\frac{1}{\lambda_{n}} F_{\boldsymbol{\psi}, n}\right] \\
& \boldsymbol{\psi}_{n x}=\frac{1}{\bar{U}_{0}^{2}-N^{2} / \lambda_{n}^{2}}\left[\frac{N^{2}}{\lambda_{n}}\left(f+\bar{U}_{0} \Lambda\right) \boldsymbol{v}_{n}+\frac{N^{2}}{\lambda_{n}} F_{\boldsymbol{u}, n}+\bar{U}_{0} F_{\boldsymbol{\psi}, n}\right] .
\end{aligned}
$$


Following the similar idea as for $\boldsymbol{v}_{x}$, we can show that $\boldsymbol{u}_{x}$ and $\boldsymbol{\psi}_{x}$ belong to $\mathbf{L}^{2}\left(\mathcal{M}^{\prime}\right)$, and

$$
\begin{aligned}
& \boldsymbol{u}_{x}^{j} \longrightarrow \boldsymbol{u}_{x} \quad \text { in } \mathbf{L}^{2}\left(\mathcal{M}^{\prime}\right), \\
& \boldsymbol{\psi}_{x}^{j} \longrightarrow \boldsymbol{\psi}_{x} \quad \text { in } \mathbf{L}^{2}\left(\mathcal{M}^{\prime}\right) .
\end{aligned}
$$

To finish the proof it remains to check that $U \in D(A)$ and $A U=F$. It is implied in the argument above that for each mode,

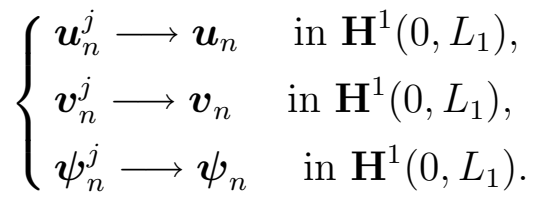

By the Sobolev embedding theorem, the convergences also hold in the space $\boldsymbol{C}\left(\left[0, L_{1}\right]\right)$, and thus the boundary conditions pass to the limit. Hence $U \in D(A)$. We infer from (3.34), (3.39), (3.42), and (3.43) that $A U^{j} \longrightarrow A U$ in $H$. By (3.33), we have $A U=F$. This completes the proof of Lemma 3.2.

Lemma 3.3. $A^{*}$ is a closed operator.

Proof. This is a consequence of Lemma 3.2 and of the following standard lemma, which can be found in Rudin (1991) and other functional analysis books.

Lemma 3.4. If $T$ is a densely defined operator in $H$, then $T^{*}$ is a closed operator.

We now state a well-known result and give a direct proof for the convenience of the reader (see Hille and Phillips (1974) and, for Banach spaces, see Brézis (1970)):

Lemma 3.5. Let $A$ and $A^{*}$ be linear unbounded operators in $H$ with domains $D(A)$ and $D\left(A^{*}\right)$ respectively, and let $A^{*}$ be the adjoint operator of $A$ (as an unbounded operator). It is also assumed that both $D(A)$ and $D\left(A^{*}\right)$ are dense in $H$. If furthermore $A$ and $A^{*}$ are both positive and closed, then $A+\mu_{0} I$ and $A^{*}+\mu_{0} I$ are onto for every $\mu_{0}>0$.

Proof. Consider $\epsilon>0$, which will eventually converge to zero. For each value of $\epsilon$, we construct a bilinear form $b_{\epsilon}$ on $D(A)$ :

$$
b_{\epsilon}(U, \widetilde{U})=\epsilon(A U, A \widetilde{U})_{H}+(A U, \widetilde{U})_{H}+\mu_{0}(U, \widetilde{U})_{H} .
$$

It is easy to check that $b_{\epsilon}$ is bilinear, bounded and coercive on $D(A)$. Then, by the Lax-Milgram theorem, for any given $F \in H$, there exists 
a unique $U_{\epsilon} \in D(A)$, such that

$$
\epsilon\left(A U_{\epsilon}, A \widetilde{U}\right)_{H}+\left(A U_{\epsilon}, \widetilde{U}\right)_{H}+\mu_{0}\left(U_{\epsilon}, \widetilde{U}\right)_{H}=(F, \widetilde{U})_{H}
$$

holds for any $\widetilde{U} \in D(A)$. For each $\epsilon$, we observe that

$$
\widetilde{U} \longrightarrow\left(A U_{\epsilon}, A \widetilde{U}\right)_{H}=\frac{1}{\epsilon}\left(F-\mu_{0} U_{\epsilon}-A U_{\epsilon}, \widetilde{U}\right)_{H}
$$

is a linear functional on $D(A)$ continuous for the norm of $H$. By the definition of the domain $D\left(A^{*}\right)$ of $A^{*}$ (see e.g. Rudin (1991)), this means that

$$
A U_{\epsilon} \in D\left(A^{*}\right)
$$

and

$$
A^{*} A U_{\epsilon}=\frac{1}{\epsilon}\left(F-\mu_{0} U_{\epsilon}-A U_{\epsilon}\right) \text { in } H .
$$

We then write (3.49) as

$$
\epsilon A^{*} A U_{\epsilon}+A U_{\epsilon}+\mu_{0} U_{\epsilon}=F .
$$

Multiplying (3.50) by $U_{\epsilon}$, we obtain

$$
\epsilon\left(A U_{\epsilon}, A U_{\epsilon}\right)_{H}+\left(A U_{\epsilon}, U_{\epsilon}\right)_{H}+\mu_{0}\left(U_{\epsilon}, U_{\epsilon}\right)_{H}=\left(F, U_{\epsilon}\right)_{H} .
$$

Since $\left(A U_{\epsilon}, A U_{\epsilon}\right)_{H} \geq 0$, and $\left(A U_{\epsilon}, U_{\epsilon}\right)_{H} \geq 0$ by the assumption that $A$ is positive, we then have

$$
\left|U_{\epsilon}\right|_{H} \leq c|F|_{H},
$$

where $c$ is a constant independent of $\epsilon$. Therefore there exists a subsequence $\epsilon^{\prime} \longrightarrow 0$ such that

$$
U_{\epsilon^{\prime}} \rightarrow U \text { weakly in } H,
$$

for some $U \in H$. Multiplying (3.50) by $A U_{\epsilon}$, we also obtain

Since $\left(A^{*} A U_{\epsilon}, A U_{\epsilon}\right)_{H} \geq 0$, and $\left(A U_{\epsilon}, U_{\epsilon}\right)_{H} \geq 0$, we have

$$
\left|A U_{\epsilon}\right|_{H} \leq|F|_{H} \text {. }
$$

This implies that there exists a subsequence, still denoted $\epsilon^{\prime}$, such that

$$
A U_{\epsilon^{\prime}} \rightarrow \chi \quad \text { weakly in } H
$$

for some $\chi \in H$. By the assumption that the operator $A$ is closed, and by (3.53) and (3.56), we see that

$$
U \in D(A) \quad \text { and } \quad \chi=A U .
$$

We find from (3.50) that

$$
A^{*}\left(\epsilon A U_{\epsilon}\right)=F-A U_{\epsilon}-\mu_{0} U_{\epsilon} .
$$


Since both $A U_{\epsilon}$ and $U_{\epsilon}$ converge weakly in $H$,

$$
A^{*}\left(\epsilon A U_{\epsilon}\right) \rightarrow \sigma=F-A U-\mu_{0} U \text { weakly in } H .
$$

And since $\left|A U_{\epsilon}\right|_{H}$ is bounded independently of $\epsilon$, we find that $\sigma=0$, that is,

$$
\left(A+\mu_{0} I\right) U=F .
$$

Thus the claim that $A+\mu_{0} I$ is onto for any $\mu_{0}>0$ is proved. That $A^{*}+\mu_{0} I$ is onto for any $\mu_{0}>0$ can be proved in a similar way.

Proof of Theorem 3.5. Let

$$
U=e^{\delta t} U^{b}
$$

where $\delta$ is the positive constant chosen in the proof of Lemma 3.1. Inserting (3.61) into (3.19), we obtain an initial value problem for $U^{b}$ :

$$
\left\{\begin{array}{l}
\frac{\mathrm{d} U^{b}}{\mathrm{~d} t}+(A+\delta) U^{b}=\widetilde{F}, \\
U^{b}(0)=U_{0} .
\end{array}\right.
$$

If we can show that the system (3.62) is well-posed, and $U^{b}$ satisfies (3.26), then, by the relation (3.61), $U$ satisfies (3.26) too, and of course (3.19) is well-posed. We have in fact verified the hypotheses of Theorem 2.1 in Lemmas 3.1 to 3.5 for the operator $A+\delta I$ of Theorem 3.5. Now we readily apply Theorem 2.1 and complete the proof of Theorem 3.5.

3.3.4. Treatment of the Constant Mode $(n=0)$. We now introduce (propose) the boundary conditions for the zero mode, which is important because it contains much energy. What follows is valid whether the boundary conditions are homogeneous or not for the modes $n \geq 1$.

We start with a technical point which has no mathematical relevance, especially for the linearized equations for which the modes are decoupled; it has however a computational and physical importance, in particular in the nonlinear case when all the modes are coupled: the function $\phi_{0}$ will be decomposed in the sum

$$
\phi_{0}=\bar{\phi}_{0}+\phi_{0}^{\prime}
$$

where $\bar{\phi}_{0}$, which is not unique, is one of the constant solutions ${ }^{13}$ of

$$
\Lambda \bar{\phi}_{0}=-\boldsymbol{f}_{0},
$$

\footnotetext{
${ }^{13} \operatorname{Det} \Lambda=0$, and $\Lambda$ is of $\operatorname{rank} 2$.
} 
with $\Lambda$ and $\boldsymbol{f}_{0}$ defined in (3.8) and (3.13) respectively, while $\boldsymbol{\phi}_{0}^{\prime}$ needs to be determined. ${ }^{14}$

Then for $\boldsymbol{u}_{0}, \boldsymbol{v}_{0}$ and $\phi_{0}^{\prime}$ we propose the following boundary conditions:

$$
\left\{\begin{array}{l}
\boldsymbol{u}_{0}(0, t)=\boldsymbol{u}_{l}(t), \\
\boldsymbol{v}_{0}(0, t)=\boldsymbol{v}_{l}(t), \\
\boldsymbol{\phi}_{0}^{\prime}(0, t)=\phi_{l}^{\prime}(t) .
\end{array}\right.
$$

Of course the third equation in (3.65) is the same as $\phi_{0}(0, t)=\phi_{l}(t)=$ $\bar{\phi}_{0}+\phi_{l}^{\prime}(t)$. We can obtain $\boldsymbol{v}_{0 x}(0, t)$ from $(3.12)_{2}$, that is

$$
\boldsymbol{v}_{0 x}(0, t)=-\frac{1}{\bar{U}_{0}}\left(\boldsymbol{v}_{l t}+f \boldsymbol{u}_{l}+\Lambda \phi_{l}^{\prime}\right) .
$$

We then multiply $(3.12)_{1}$ by $\Lambda$,

$$
\left(\Lambda \boldsymbol{u}_{0}\right)_{t}+\bar{U}_{0}\left(\Lambda \boldsymbol{u}_{0}\right)_{x}+\Lambda \phi_{0 x}^{\prime}-f \Lambda \boldsymbol{v}_{0}=0
$$

and differentiate $(3.12)_{2}$ with respect to $x$,

$$
\left(\boldsymbol{v}_{0 x}\right)_{t}+\bar{U}_{0} \boldsymbol{v}_{0 x x}+f \boldsymbol{u}_{0 x}+\Lambda \phi_{0 x}^{\prime}=0
$$

By subtracting (3.68) from (3.67) we obtain, thanks to equation $(3.12)_{3}$,

$$
\left(\Lambda \boldsymbol{u}_{0}-\boldsymbol{v}_{0 x}\right)_{t}+\bar{U}_{0}\left(\Lambda \boldsymbol{u}_{0}-\boldsymbol{v}_{0 x}\right)_{x}=0 .
$$

The value of $\Lambda \boldsymbol{u}_{0}-\boldsymbol{v}_{0 x}$ at $x=0$ is known, and therefore we can solve the equation above for $\Lambda \boldsymbol{u}_{0}-\boldsymbol{v}_{0 x}$. Once we have found $\Lambda \boldsymbol{u}_{0}-\boldsymbol{v}_{0 x}$, say $\Lambda \boldsymbol{u}_{0}-\boldsymbol{v}_{0 x}=k(x, t)$, then with $(3.12)_{3}$ we have

$$
\left\{\begin{array}{l}
\boldsymbol{u}_{0 x}+\Lambda \boldsymbol{v}_{0}=0, \\
\boldsymbol{v}_{0 x}-\Lambda \boldsymbol{u}_{0}=-k(x, t) .
\end{array}\right.
$$

We can solve this linear ODE system with the boundary conditions for $\boldsymbol{u}_{0}$ and $\boldsymbol{v}_{0}$ at $x=0$, which are given.

We now have $\boldsymbol{u}_{0}$ and $\boldsymbol{v}_{0}$; we can solve $(3.12)_{1}$ for $\boldsymbol{\phi}_{0}=\bar{\phi}_{0}+\boldsymbol{\phi}_{0}^{\prime}$, since the boundary condition for $\phi_{0}\left(\right.$ or $\left.\phi_{0}{ }^{\prime}\right)$ is also given at $x=0$.

We leave as an exercise to the reader to find the suitable regularity assumptions for the data $\boldsymbol{u}_{l}, \boldsymbol{v}_{l}$ and $\phi_{l}^{\prime}$.

\footnotetext{
${ }^{14}$ As we observed, $\Lambda$ is essentially the mathematical representation of $\partial / \partial y$ in the finite-element Galerkin procedure, and $\bar{\phi}_{0}$ is the part of the basic geostrophic flow alluded to in (3.1).
} 
3.3.5. The Case of Nonhomogeneous Boundary Conditions. In practical simulations we want to be able to solve the Primitive Equations (3.7) with nonhomogeneous boundary conditions at $x=0$ and $L_{1}$. We write (3.7) in a form similar to (3.19) corresponding to the elimination of $\boldsymbol{w}$ and $\boldsymbol{\phi}$ and the exclusion of the zero mode:

$$
\left\{\begin{array}{l}
\frac{\partial U}{\partial t}+\mathcal{A} U=F \\
U(t=0)=U_{0}
\end{array}\right.
$$

Here $U=(\boldsymbol{u}, \boldsymbol{v}, \boldsymbol{\psi})$ and $F=\left(F_{\boldsymbol{u}}, F_{\boldsymbol{v}}, F_{\boldsymbol{\psi}}\right)$ are like before, and $\mathcal{A}$ is the differential operator represented by the right-hand side of (3.25) or (3.27). The proposed boundary conditions for $U$ at $x=0$ and $L_{1}$ will be derived from given functions $U^{g, l}(z, t)$ and $U^{g . r}(z, t)$. In this subsection we will demonstrate how to derive from $U^{g, l}$ and $U^{g, r}$ the boundary conditions for $U$ so that the initial boundary value problem corresponding to (3.71) is well-posed.

As pointed out in Section 3.2.3, the subcritical and the supercritical modes require different boundary conditions. From the physical and computational points of view, we can assume that all the components of $U^{g, l}$ and $U^{g, r}$ are available. The mathematical issue is then to determine which components are needed for each mode. The normal mode expansions for $U^{g, l}$ and $U^{g, r}$ are given:

$$
\left\{\begin{array}{l}
U^{g, l}(z, t)=\left(\sum_{n \geq 0} \boldsymbol{u}_{n}^{g, l}(t) \mathcal{U}_{n}(z), \sum_{n \geq 0} \boldsymbol{v}_{n}^{g, l}(t) \mathcal{U}_{n}(z), \sum_{n \geq 1} \boldsymbol{\psi}_{n}^{g, l}(t) \mathcal{W}_{n}(z)\right) \\
U^{g, r}(z, t)=\left(\sum_{n \geq 0} \boldsymbol{u}_{n}^{g, r}(t) \mathcal{U}_{n}(z), \sum_{n \geq 0} \boldsymbol{v}_{n}^{g, r}(t) \mathcal{U}_{n}(z), \sum_{n \geq 1} \boldsymbol{\psi}_{n}^{g, r}(t) \mathcal{W}_{n}(z)\right) .
\end{array}\right.
$$

From $U^{g, l}$ and $U^{g, r}$ we construct $U^{g}=U^{g}(z, t)$,

$$
U^{g}(z, t)=\left(\sum_{n \geq 1} \boldsymbol{u}_{n}^{g}(t) \mathcal{U}_{n}(z), \sum_{n \geq 1} \boldsymbol{v}_{n}^{g}(t) \mathcal{U}_{n}(z), \sum_{n \geq 1} \boldsymbol{\psi}_{n}^{g}(t) \mathcal{W}_{n}(z)\right)
$$

where, for each $n \geq 1, \boldsymbol{u}_{n}^{g}, \boldsymbol{v}_{n}^{g}$ and $\boldsymbol{\psi}_{n}^{g}$ are determined by the following equations:

$$
\left\{\begin{array}{l}
\boldsymbol{u}_{n}^{g}(t)-\frac{1}{N} \boldsymbol{\psi}_{n}^{g}(t)=\boldsymbol{u}_{n}^{g, l}(t)-\frac{1}{N} \boldsymbol{\psi}_{n}^{g, l}(t), \\
\boldsymbol{v}_{n}^{g}(t)=\boldsymbol{v}_{n}^{g, l}(t), \\
\boldsymbol{u}_{n}^{g}(t)+\frac{1}{N} \boldsymbol{\psi}_{n}^{g}(t)=\boldsymbol{u}_{n}^{g, r}(t)+\frac{1}{N} \boldsymbol{\psi}_{n}^{g, r}(t),
\end{array} \text { if } 1 \leq n \leq n_{c},\right.
$$


that is

$$
\left\{\begin{array}{l}
\boldsymbol{u}_{n}^{g}(t)=\frac{1}{2}\left(\boldsymbol{u}_{n}^{g, l}(t)+\boldsymbol{u}_{n}^{g, r}(t)\right)+\frac{1}{2 N}\left(\boldsymbol{\psi}_{n}^{g, r}(t)-\boldsymbol{\psi}_{n}^{g, l}(t)\right) \\
\boldsymbol{v}_{n}^{g}(t)=\boldsymbol{v}_{n}^{g, l}(t), \\
\boldsymbol{\psi}_{n}^{g}(t)=\frac{N}{2}\left(\boldsymbol{u}_{n}^{g, r}(t)-\boldsymbol{u}_{n}^{g, l}\right)(t)+\frac{1}{2}\left(\boldsymbol{\psi}_{n}^{g, l}(t)+\boldsymbol{\psi}_{n}^{g, r}(t)\right),
\end{array}\right.
$$

and

$$
\left\{\begin{array}{l}
\boldsymbol{u}_{n}^{g}(t)=\boldsymbol{u}_{n}^{g, l}(t), \\
\boldsymbol{v}_{n}^{g}(t)=\boldsymbol{v}_{n}^{g, l}(t), \quad \text { if } n>n_{c} \\
\boldsymbol{\psi}_{n}^{g}(t)=\boldsymbol{\psi}_{n}^{g, l}(t) .
\end{array}\right.
$$

We observe here that $U^{g}$ is independent of $x$, i.e., $\partial U^{g} / \partial x=0$, and that, for a.e. $t \in(0, T), U^{g} \in H$ provided that $U^{g, l}$ and $U^{g, r}$ are sufficiently smooth: indeed $U^{g} \in \mathbf{L}^{2}\left(\mathcal{M}^{\prime}\right)^{3}$, and the integral conditions appearing in (3.20) are automatically satisfied since the mode 0 is not present here $(n \geq 1)$.

Then we set

$$
U=U^{\#}+U^{g}
$$

We observe that $U^{\#} \in D(A)$ (if $U^{\#}$ is sufficiently smooth). Then setting $F^{\#}=F-\partial U^{g} / \partial t-\mathcal{A} U^{g}$ and $U_{0}^{\#}=U_{0}-\left.U^{g}\right|_{t=0}$, we see that $U^{\#}$ is the solution of the following problem:

$$
\left\{\begin{array}{l}
\frac{\mathrm{d} U^{\#}}{\mathrm{~d} t}+A U^{\#}=F^{\#} \\
U^{\#}(t=0)=U_{0}^{\#}
\end{array}\right.
$$

Like (3.19), (3.78) corresponds to the case with homogeneous boundary conditions. In order to apply Theorem 3.5 to (3.78) we would need to have

$$
U_{0}^{\#}=U_{0}-\left.U^{g}\right|_{t=0} \in D(A)
$$

and

$$
F^{\#}, \frac{\mathrm{d} F^{\#}}{\mathrm{~d} t} \in L^{1}\left(0, T ; \mathbf{L}^{2}(\mathcal{M})^{3}\right)
$$


It is easily seen that (3.79) and (3.80) are satisfied if the following hypotheses are verified (up to (3.85), and see also (3.89)):

$$
\begin{gathered}
U_{0} \in \mathbf{L}^{2}(\mathcal{M})^{3}, \quad \frac{\partial U_{0}}{\partial x} \in \mathbf{L}^{2}(\mathcal{M})^{3} \\
F \in L^{1}\left(0, T ; \mathbf{L}^{2}(\mathcal{M})^{3}\right), \quad \frac{\partial F}{\partial t} \in L^{1}\left(0, T ; \mathbf{L}^{2}(\mathcal{M})^{3}\right), \\
\frac{\partial^{k} U^{g, l}}{\partial t^{k}}, \frac{\partial^{k} U^{g, r}}{\partial t^{k}} \in L^{1}\left(0, T ; \mathbf{L}^{2}\left(-L_{3}, 0\right)^{3}\right) \quad \text { for } k=0,1,2 .
\end{gathered}
$$

In addition we must assume that $U_{0}, U^{g, l}$ and $U^{g, r}$ satisfy the compatibility conditions which will guarantee that the boundary conditions included in (3.79) are satisfied. Denoting the function $U_{0}$ of initial values by $\left(\widetilde{\boldsymbol{u}}_{0}, \widetilde{\boldsymbol{v}}_{0}, \widetilde{\boldsymbol{\psi}}_{0}\right),{ }^{15}$ the compatibility conditions for $U_{0}, U^{g, l}$ and $U^{g, r}$ which guarantee that $U_{0}^{\#} \in D(A)$ are written:

$$
\left\{\begin{array}{l}
\int_{-L_{3}}^{0} \widetilde{\boldsymbol{u}}_{0}(x=0, z) \mathcal{U}_{n}(z)-\frac{1}{N} \int_{-L_{3}}^{0} \widetilde{\boldsymbol{\psi}}_{0}(x=0, z) \mathcal{W}_{n}(z) \mathrm{d} z \\
=\int_{-L_{3}}^{0} \boldsymbol{u}^{g, l}(z, t=0) \mathcal{U}_{n}(z)-\frac{1}{N} \int_{-L_{3}}^{0} \boldsymbol{\psi}^{g, l}(z, t=0) \mathcal{W}_{n}(z) \mathrm{d} z \\
\int_{-L_{3}}^{0} \widetilde{\boldsymbol{v}}_{0}(x=0, z) \mathcal{U}_{n}(z) \mathrm{d} z=\int_{-L_{3}}^{0} \boldsymbol{v}^{g, l}(z, t=0) \mathcal{U}_{n}(z) \mathrm{d} z, \quad \text { for } 1 \leq n \leq n_{c} \\
\int_{-L_{3}}^{0} \widetilde{\boldsymbol{u}}_{0}\left(x=L_{1}, z\right) \mathcal{U}_{n}(z)+\frac{1}{N} \int_{-L_{3}}^{0} \widetilde{\boldsymbol{\psi}}_{0}\left(x=L_{1}, z\right) \mathcal{W}_{n}(z) \mathrm{d} z \\
=\int_{-L_{3}}^{0} \boldsymbol{u}^{g, r}(z, t=0) \mathcal{U}_{n}(z)+\frac{1}{N} \int_{-L_{3}}^{0} \boldsymbol{\psi}^{g, r}(z, t=0) \mathcal{W}_{n}(z) \mathrm{d} z
\end{array}\right.
$$

and

$$
\left\{\begin{array}{l}
\int_{-L_{3}}^{0} \widetilde{\boldsymbol{u}}_{0}(x=0, z) \mathcal{U}_{n}(z) \mathrm{d} z=\int_{-L_{3}}^{0} \boldsymbol{u}^{g, l}(z, t=0) \mathcal{U}_{n}(z) \mathrm{d} z \\
\int_{-L_{3}}^{0} \widetilde{\boldsymbol{v}}_{0}(x=0, z) \mathcal{U}_{n}(z) \mathrm{d} z=\int_{-L_{3}}^{0} \boldsymbol{v}^{g, l}(z, t=0) \mathcal{U}_{n}(z) \mathrm{d} z, \quad \text { for } n>n_{c} \\
\int_{-L_{3}}^{0} \widetilde{\boldsymbol{\psi}}_{0}(x=0, z) \mathcal{W}_{n}(z) \mathrm{d} z=\int_{-L_{3}}^{0} \boldsymbol{\psi}^{g, l}(z, t=0) \mathcal{W}_{n}(z) \mathrm{d} z
\end{array}\right.
$$

\footnotetext{
${ }^{15}$ The tildes here on $\widetilde{\boldsymbol{u}}_{0}, \widetilde{\boldsymbol{v}}_{0}$ and $\widetilde{\boldsymbol{\psi}}_{0}$ are meant to distinguish these initial datas from the zero modes of $U(t)$, which do not appear in fact in this subsection.
} 
It should be noted here that (3.81)-(3.85) are sufficient conditions for (3.79) and (3.80). They have been chosen for their relative simplicity; (3.84) and (3.85) indeed guarantee that the boundary conditions required in (3.79) $\left(U_{0}^{\#} \in D(A)\right)$ are satisfied.

Now we can apply Theorem 3.5 to the system (3.78), and we obtain a unique solution $U^{\#}$ that satisfies the analogue of (3.26). We then recover $U$ via (3.77), and we easily see that

$$
\begin{aligned}
U & \in \mathcal{C}\left([0, T] ; \mathbf{L}^{2}(\mathcal{M})^{3}\right), \\
\frac{\partial U}{\partial x} & \in L^{\infty}\left(0, T ; \mathbf{L}^{2}(\mathcal{M})^{3}\right) .
\end{aligned}
$$

We will also have

$$
\frac{\partial U}{\partial t} \in L^{\infty}\left(0, T ; \mathbf{L}^{2}(\mathcal{M})^{3}\right)
$$

provided we further require that

$$
\frac{\partial U^{g, l}}{\partial t}, \frac{\partial U^{g, r}}{\partial t} \in L^{\infty}\left(0, T ; \mathbf{L}^{2}\left(-L_{3}, 0\right)^{3}\right)
$$

The boundary conditions that $U$ satisfies, expressing the fact that $U^{\#}(t)=U(t)-U^{g}(t)$ belongs to $D(A)$ for a.e. $\mathrm{t}$, are as follows:

For the subcritical modes $1 \leq n \leq n_{c}$ :

$$
\left\{\begin{array}{l}
\int_{-L_{3}}^{0} \boldsymbol{u}(0, z, t) \mathcal{U}_{n}(z)-\frac{1}{N} \int_{-L_{3}}^{0} \boldsymbol{\psi}(0, z, t) \mathcal{W}_{n}(z) \mathrm{d} z \\
\quad=\int_{-L_{3}}^{0} \boldsymbol{u}^{g, l}(z, t) \mathcal{U}_{n}(z)-\frac{1}{N} \int_{-L_{3}}^{0} \boldsymbol{\psi}^{g, l}(z, t) \mathcal{W}_{n}(z) \mathrm{d} z \\
\int_{-L_{3}}^{0} \boldsymbol{v}(0, z, t) \mathcal{U}_{n}(z) \mathrm{d} z=\int_{-L_{3}}^{0} \boldsymbol{v}^{g, l}(z, t) \mathcal{U}_{n}(z) \mathrm{d} z \\
\int_{-L_{3}}^{0} \boldsymbol{u}\left(L_{1}, z, t\right) \mathcal{U}_{n}(z)+\frac{1}{N} \int_{-L_{3}}^{0} \boldsymbol{\psi}\left(L_{1}, z, t\right) \mathcal{W}_{n}(z) \mathrm{d} z \\
=\int_{-L_{3}}^{0} \boldsymbol{u}^{g, r}(z, t) \mathcal{U}_{n}(z)+\frac{1}{N} \int_{-L_{3}}^{0} \boldsymbol{\psi}^{g, r}(z, t) \mathcal{W}_{n}(z) \mathrm{d} z
\end{array}\right.
$$


and for the supercritical mode $n>n_{c}$ :

$$
\left\{\begin{array}{l}
\int_{-L_{3}}^{0} \boldsymbol{u}(0, z, t) \mathcal{U}_{n}(z) \mathrm{d} z=\int_{-L_{3}}^{0} \boldsymbol{u}^{g, l}(z, t) \mathcal{U}_{n}(z) \mathrm{d} z \\
\int_{-L_{3}}^{0} \boldsymbol{v}(0, z, t) \mathcal{U}_{n}(z) \mathrm{d} z=\int_{-L_{3}}^{0} \boldsymbol{v}^{g, l}(z, t) \mathcal{U}_{n}(z) \mathrm{d} z \\
\int_{-L_{3}}^{0} \boldsymbol{\psi}(0, z, t) \mathcal{W}_{n}(z) \mathrm{d} z=\int_{-L_{3}}^{0} \boldsymbol{\psi}^{g, l}(z, t) \mathcal{W}_{n}(z) \mathrm{d} z
\end{array}\right.
$$

We summarize the result concerning the case of nonhomogeneous boundary conditions in a theorem.

Theorem 3.6. Let $H$ be the Hilbert space defined in (3.20), A the linear operator defined in (3.25), $\mathcal{A}$ the corresponding differential operator, and let $D(A)$ be the domain of the operator $A$ in $H$. We assume that the data $U_{0}, F, U^{g, l}$ and $U^{g, r}$ satisfy the regularity conditions (3.81)-(3.83), and in addition $U_{0}, U^{g, l}$ and $U^{g, r}$ satisfy the compatibility conditions (3.84) and (3.85). Then the initial boundary value problem corresponding to (3.71) supplemented with the boundary conditions (3.90) and (3.91) has a unique solution $U$, and $U$ satisfies (3.86) and (3.87); $U$ will also satisfy (3.88) if we furthermore assume (3.89) for $U^{g, l}$ and $U^{g, r}$.

3.4. Special Case of Impenetrable Boundaries. We now consider another interesting model with only one degree of freedom for $v$ (one unknown component for $v$ ). In this case we require that $v$ vanishes at $y=0$ and 1 , which in physics corresponds to impenetrable boundaries at the North and South. To impose this boundary condition, we use a single mode in the $y$-direction for $v$, namely $\widetilde{h}_{2}$ (see Fig. 3.2.1), and the other unknowns $u, w, \phi$ and $\psi$ are decomposed as they were in Section 3.2.1; hence:

$$
\left\{\begin{array}{l}
u \simeq u_{1}(x, z, t) h_{1}(y)+u_{2}(x, z, t) h_{2}(y)+u_{3}(x, z, t) h_{3}(y) \\
v \simeq v_{2}(x, z, t) \widetilde{h}_{2}(y) \\
w \simeq w_{1}(x, z, t) h_{1}(y)+w_{2}(x, z, t) h_{2}(y)+w_{3}(x, z, t) h_{3}(y) \\
\phi \simeq \phi_{1}(x, z, t) h_{1}(y)+\phi_{2}(x, z, t) h_{2}(y)+\phi_{3}(x, z, t) h_{3}(y) \\
\psi \simeq \psi_{1}(x, z, t) h_{1}(y)+\psi_{2}(x, z, t) h_{2}(y)+\psi_{3}(x, z, t) h_{3}(y)
\end{array}\right.
$$

Then we introduce (3.92) into (3.3). We perform the same operations as after $(3.3)$, except that we multiply the equation for $v,(3.3)_{2}$, by $\widetilde{h}_{2}$, integrate over $(0,1)$, and divide it by $\int_{0}^{1} \widetilde{h}_{2}^{2} d y$. Thus we arrive at the 
following approximation of the system (3.3):

$$
\left\{\begin{array}{l}
\boldsymbol{u}_{t}+\bar{U}_{0} \boldsymbol{u}_{x}+\boldsymbol{\phi}_{x}+f v_{2} \boldsymbol{\sigma}_{\mathbf{1}}=0 \\
v_{2 t}+\bar{U}_{0} v_{2 x}+f \boldsymbol{\sigma}_{\mathbf{3}} \cdot \boldsymbol{u}+\boldsymbol{\sigma}_{\mathbf{4}} \cdot \boldsymbol{\phi}+\frac{3}{2} f \bar{U}_{0}=0 \\
\boldsymbol{\psi}_{t}+\bar{U}_{0} \boldsymbol{\psi}_{x}+N^{2} \boldsymbol{w}=0 \\
\boldsymbol{u}_{x}+v_{2} \boldsymbol{\sigma}_{\mathbf{2}}+\boldsymbol{w}_{z}=0 \\
\boldsymbol{\psi}=\boldsymbol{\phi}_{z}
\end{array}\right.
$$

The vector notation, i.e. $\boldsymbol{u}=\left(u_{1}, u_{2}, u_{3}\right), \boldsymbol{w}=\left(w_{1}, w_{2}, w_{3}\right)$ etc., has been used, and

$$
\boldsymbol{\sigma}_{1}=\frac{1}{2}\left(\begin{array}{c}
-1 \\
1 \\
-1
\end{array}\right), \quad \sigma_{2}=3\left(\begin{array}{c}
1 \\
0 \\
-1
\end{array}\right), \quad \sigma_{3}=\frac{1}{4}\left(\begin{array}{c}
1 \\
-6 \\
1
\end{array}\right), \quad \sigma_{4}=\frac{3}{2}\left(\begin{array}{c}
-1 \\
0 \\
1
\end{array}\right) .
$$

The dot in (3.93) represents the dot product in the Euclidean space. We note here that all the equations in (3.93) are vector equations except the second one, which is a scalar equation for the scalar unknown $v_{2}$.

From here on, we proceed essentially as in Sections 3.2.2, 3.2.3 and Section 3.3, and below we only highlight the differences with the previous case.

The normal mode expansion for $\boldsymbol{u}, \boldsymbol{w}, \boldsymbol{\phi}, \boldsymbol{\psi}$ are the same as before, and for $v_{2}$ it reads

$$
v_{2}(x, z, t)=\sum_{n \geq 0} v_{2 n}(x, t) \mathcal{U}_{n}(z) .
$$

The system for the zero mode is

$$
\left\{\begin{array}{l}
\boldsymbol{u}_{0 t}+\bar{U}_{0} \boldsymbol{u}_{0 x}+\phi_{0 x}+f v_{20} \boldsymbol{\sigma}_{\mathbf{1}}=0 \\
v_{20 t}+\bar{U}_{0} v_{20 x}+f \boldsymbol{\sigma}_{\mathbf{3}} \cdot \boldsymbol{u}_{0}+\boldsymbol{\sigma}_{\mathbf{4}} \cdot \boldsymbol{\phi}_{0}+\frac{3}{2} L_{3}^{\frac{1}{2}} f \bar{U}_{0}=0 \\
\boldsymbol{u}_{0 x}+v_{20} \boldsymbol{\sigma}_{\mathbf{2}}=0
\end{array}\right.
$$

For $n \geq 1$ the system is

$$
\left\{\begin{array}{l}
\boldsymbol{u}_{n t}+\bar{U}_{0} \boldsymbol{u}_{n x}+\boldsymbol{\phi}_{n x}+f v_{2 n} \boldsymbol{\sigma}_{\mathbf{1}}=0 \\
v_{2 n t}+\bar{U}_{0} v_{2 n x}+f \boldsymbol{\sigma}_{\mathbf{3}} \cdot \boldsymbol{u}_{n}+\boldsymbol{\sigma}_{\mathbf{4}} \cdot \boldsymbol{\phi}_{n}=0 \\
\boldsymbol{\psi}_{n t}+\bar{U}_{0} \boldsymbol{\psi}_{n x}+N^{2} \boldsymbol{w}_{n}=0 \\
\boldsymbol{u}_{n x}+v_{2 n} \boldsymbol{\sigma}_{\mathbf{2}}+\lambda_{n} \boldsymbol{w}_{n}=0 \\
-\lambda_{n} \boldsymbol{\phi}_{n}=\boldsymbol{\psi}_{n}
\end{array}\right.
$$


Eliminating $\boldsymbol{\phi}_{n}$ and $\boldsymbol{w}_{n}$ from (3.97) we obtain a system for $\boldsymbol{u}_{n}, \boldsymbol{v}_{n}$ and $\boldsymbol{\psi}_{n}(n \geq 1)$, namely:

$$
\left\{\begin{array}{l}
\boldsymbol{u}_{n t}+\bar{U}_{0} \boldsymbol{u}_{n x}-\frac{1}{\lambda_{n}} \boldsymbol{\psi}_{n x}+f v_{2 n} \boldsymbol{\sigma}_{\mathbf{1}}=0, \\
\boldsymbol{v}_{n t}+\bar{U}_{0} \boldsymbol{v}_{n x}+f \boldsymbol{\sigma}_{\mathbf{3}} \boldsymbol{u}_{n}-\frac{1}{\lambda_{n}} \boldsymbol{\sigma}_{\mathbf{4}} \cdot \boldsymbol{\psi}_{n}=0, \\
\boldsymbol{\psi}_{n t}-\frac{N^{2}}{\lambda_{n}} \boldsymbol{u}_{n x}+\bar{U}_{0} \boldsymbol{\psi}_{n x}-\frac{N^{2}}{\lambda_{n}} v_{2 n} \boldsymbol{\sigma}_{\mathbf{2}}=0 .
\end{array}\right.
$$

The coefficient matrix associated with the first order derivative terms (with respect to $x$ ) terms in the first and third equations in (3.98) is

$$
\left(\begin{array}{cc}
\bar{U}_{0} & -\frac{1}{\lambda_{n}} \\
-\frac{N^{2}}{\lambda_{n}} & \bar{U}_{0}
\end{array}\right)
$$

This matrix has two eigenvalues: $U_{0}+N / \lambda_{n}$ and $U_{0}-N / \lambda_{n}$. The first eigenvalue is always positive, while the second one could be positive, which corresponds to supercritical modes, or negative, which corresponds to subcritical modes. As before, we let $n_{c}$ denote the number of subcritical modes, and for each $n \geq 1$ we also introduce the variables $\boldsymbol{\xi}_{n}=\boldsymbol{u}_{n}-\boldsymbol{\psi}_{n} / N, \boldsymbol{\eta}_{n}=\boldsymbol{u}_{n}+\boldsymbol{\psi}_{n} / N$. By an analysis similar to that in Section 3.2.3 we are led to propose the following boundary conditions for the subcritical modes:

$$
\left\{\begin{array}{l}
\boldsymbol{\xi}_{n}(0, t)=0, \\
v_{2 n}(0, t)=0, \\
\boldsymbol{\eta}_{n}\left(L_{1}, t\right)=0,
\end{array} \quad \text { for } 1 \leq n \leq n_{c}\right.
$$

and, for the supercritical modes:

$$
\left\{\begin{array}{l}
\boldsymbol{\xi}_{n}(0, t)=0, \\
v_{2 n}(0, t)=0, \\
\boldsymbol{\eta}_{n}(0, t)=0 .
\end{array} \quad \text { for } n>n_{c} .\right.
$$

Again we want to transform (3.93) (except the zero mode, which needs a separate treatment) into an abstract initial value problem of the form

$$
\left\{\begin{array}{l}
\frac{\mathrm{d} U}{\mathrm{~d} t}+A U=F, \\
U(0)=U_{0} .
\end{array}\right.
$$

For this purpose we introduce the following function spaces:

$$
H=H_{\boldsymbol{u}} \times H_{v_{2}} \times H_{\boldsymbol{\psi}},
$$




$$
\begin{aligned}
H_{\boldsymbol{u}} & =\left\{\boldsymbol{u} \in \mathbf{L}^{2}\left(\mathcal{M}^{\prime}\right) \mid \int_{-L_{3}}^{0} \boldsymbol{u}(x, z) \mathrm{d} z=\mathbf{0}, \text { for a.e. } x \in\left(0, L_{1}\right)\right\} \\
H_{v_{2}} & =\left\{v_{2} \in L^{2}\left(\mathcal{M}^{\prime}\right) \mid \int_{-L_{3}}^{0} v_{2}(x, z) \mathrm{d} z=0, \text { for a.e. } x \in\left(0, L_{1}\right)\right\}, \\
H_{\boldsymbol{\psi}} & =\mathbf{L}^{2}\left(\mathcal{M}^{\prime}\right) .
\end{aligned}
$$

We endow $H$ with the inner product

$$
(U, \widetilde{U})_{H}=\int_{\mathcal{M}^{\prime}}\left(\boldsymbol{u} \cdot \widetilde{\boldsymbol{u}}+v_{2} \widetilde{v_{2}}+\frac{1}{N^{2}} \boldsymbol{\psi} \cdot \widetilde{\boldsymbol{\psi}}\right) \mathrm{d} \mathcal{M}^{\prime} \quad \text { for } U, \widetilde{U} \in H
$$

With this inner product, $H$ is a Hilbert space. We let $P$ denote the orthogonal projector from $\mathbf{L}^{2}\left(\mathcal{M}^{\prime}\right)$ onto $H_{\boldsymbol{u}}$. For convenience we also use $P$ for the orthogonal projector from $L^{2}\left(\mathcal{M}^{\prime}\right)$ onto $H_{v_{2}}$.

The boundary conditions for $\boldsymbol{u}, v_{2}$ and $\boldsymbol{\psi}$ follow those we chose above, mode by mode ( see (3.99), (3.100)); hence:

For the subcritical modes $\left(1 \leq n \leq n_{c}\right)$,

$$
\left\{\begin{array}{l}
\int_{-L_{3}}^{0} \boldsymbol{u}(0, z) \mathcal{U}_{n}(z)-\frac{1}{N} \int_{-L_{3}}^{0} \boldsymbol{\psi}(0, z) \mathcal{W}_{n}(z) \mathrm{d} z=\mathbf{0} \\
\int_{-L_{3}}^{0} v_{2}(0, z) \mathcal{U}_{n}(z)=\mathbf{0} \\
\int_{-L_{3}}^{0} \boldsymbol{u}\left(L_{1}, z\right) \mathcal{U}_{n}(z)+\frac{1}{N} \int_{-L_{3}}^{0} \boldsymbol{\psi}\left(L_{1}, z\right) \mathcal{W}_{n}(z) \mathrm{d} z=\mathbf{0}
\end{array}\right.
$$

and for the supercritical modes $\left(n>n_{c}\right)$,

$$
\left\{\begin{array}{l}
\int_{-L_{3}}^{0} \boldsymbol{u}(0, z) \mathcal{U}_{n}(z) \mathrm{d} z=\mathbf{0} \\
\int_{-L_{3}}^{0} v_{2}(0, z) \mathcal{U}_{n}(z) \mathrm{d} z=\mathbf{0} \\
\int_{-L_{3}}^{0} \boldsymbol{\psi}(0, z) \mathcal{W}_{n}(z) \mathrm{d} z=\mathbf{0}
\end{array}\right.
$$

The domain of the operator $A$ is defined as

$$
\begin{aligned}
& D(A)=\left\{U \in H \mid U_{x} \in \mathbf{L}^{2}\left(\mathcal{M}^{\prime}\right) \times L^{2}\left(\mathcal{M}^{\prime}\right) \times \mathbf{L}^{2}\left(\mathcal{M}^{\prime}\right)\right. \\
&\text { and Uverifies the } \left.B C^{\prime} \text { s (3.104) and (3.105) }\right\} .
\end{aligned}
$$


For each $U \in D(A), A U$ is given by

$$
A U=\left(\begin{array}{l}
\bar{U}_{0} \boldsymbol{u}_{x}+f v_{2} \boldsymbol{\sigma}_{\mathbf{1}}-P\left[\int_{z}^{0} \boldsymbol{\psi}_{x}\left(x, z^{\prime}\right) \mathrm{d} z^{\prime}\right] \\
\bar{U}_{0} v_{2 x}+f \boldsymbol{\sigma}_{\mathbf{3}} \cdot \boldsymbol{u}-P\left[\int_{z}^{0} \boldsymbol{\sigma}_{\mathbf{4}} \cdot \boldsymbol{\psi}\left(x, z^{\prime}\right) \mathrm{d} z^{\prime}\right] \\
\bar{U}_{0} \boldsymbol{\psi}_{x}+N^{2} \int_{z}^{0}\left(\boldsymbol{u}_{x}+v_{2} \boldsymbol{\sigma}_{\mathbf{2}}\right) \mathrm{d} z^{\prime}
\end{array}\right)
$$

In the process of establishing the well-posedness of the initial value problem associated with our new model we need to determine the adjoint operator $A^{*}$ of $A$ (as an unbounded operator in $H$ ), and its domain $D\left(A^{*}\right)$. We now list, without details of the calculations, the definitions of the operator $A^{*}$ and its domain $D\left(A^{*}\right)$. The functions $\widetilde{U}=\left(\widetilde{\boldsymbol{u}}, \widetilde{v}_{2}, \widetilde{\boldsymbol{\psi}}\right)$ in $D\left(A^{*}\right)$ satisfy the following boundary conditions.

For the subcritical modes $\left(1 \leq n \leq n_{c}\right)$ :

$$
\left\{\begin{array}{l}
\int_{-L_{3}}^{0} \widetilde{\boldsymbol{u}}\left(L_{1}, z\right) \mathcal{U}_{n}(z)-\frac{1}{N} \int_{-L_{3}}^{0} \widetilde{\boldsymbol{\psi}}\left(L_{1}, z\right) \mathcal{W}_{n}(z) \mathrm{d} z=\mathbf{0} \\
\int_{-L_{3}}^{0} \widetilde{v}_{2}\left(L_{1}, z\right) \mathcal{U}_{n}(z)=\mathbf{0} \\
\int_{-L_{3}}^{0} \widetilde{\boldsymbol{u}}(0, z) \mathcal{U}_{n}(z)+\frac{1}{N} \int_{-L_{3}}^{0} \widetilde{\boldsymbol{\psi}}(0, z) \mathcal{W}_{n}(z) \mathrm{d} z=\mathbf{0}
\end{array}\right.
$$

and for the supercritical modes $\left(n>n_{c}\right)$ :

$$
\left\{\begin{array}{l}
\int_{-L_{3}}^{0} \widetilde{\boldsymbol{u}}\left(L_{1}, z\right) \mathcal{U}_{n}(z) \mathrm{d} z=\mathbf{0} \\
\int_{-L_{3}}^{0} \widetilde{v}_{2}\left(L_{1}, z\right) \mathcal{U}_{n}(z) \mathrm{d} z=\mathbf{0} \\
\int_{-L_{3}}^{0} \widetilde{\boldsymbol{\psi}}\left(L_{1}, z\right) \mathcal{W}_{n}(z) \mathrm{d} z=\mathbf{0}
\end{array}\right.
$$

The domain $D\left(A^{*}\right)$ is then defined as follows:

$$
\begin{array}{r}
D\left(A^{*}\right)=\left\{\widetilde{U} \in H \mid \widetilde{U}_{x} \in\left(\mathbf{L}^{2}\left(\mathcal{M}^{\prime}\right) \times L^{2}\left(\mathcal{M}^{\prime}\right) \times \mathbf{L}^{2}\left(\mathcal{M}^{\prime}\right),\right.\right. \\
\text { and } \left.\widetilde{U} \text { verifies the } B C^{\prime} s(3.108) \text { and }(3.109)\right\} .
\end{array}
$$


For each $\widetilde{U} \in D\left(A^{*}\right), A^{*} \widetilde{U}$ is given by

$$
A^{*} \widetilde{U}=\left(\begin{array}{c}
\sum_{n \geq 1}\left(-\bar{U}_{0} \widetilde{\boldsymbol{u}}_{n x}+\frac{1}{N \lambda_{n}} \widetilde{\boldsymbol{\psi}}_{n x}+f \widetilde{v}_{2 n} \boldsymbol{\sigma}_{\mathbf{3}}\right) \mathcal{U}_{n} \\
\sum_{n \geq 1}\left(-\bar{U}_{0} \widetilde{v}_{2 n x}+f \widetilde{\boldsymbol{u}}_{n} \cdot \boldsymbol{\sigma}_{\mathbf{1}}-\frac{1}{N \lambda_{n}} \widetilde{\boldsymbol{\psi}}_{n} \cdot \boldsymbol{\sigma}_{\mathbf{2}}\right) \mathcal{U}_{n} \\
\sum_{n \geq 1}\left(\frac{N}{\lambda_{n}} \widetilde{\boldsymbol{u}}_{n x}-\bar{U}_{0} \widetilde{\boldsymbol{\psi}}_{n x}-\frac{N}{\lambda_{n}} \widetilde{v}_{2 n} \boldsymbol{\sigma}_{\mathbf{4}}\right) \mathcal{W}_{n}
\end{array}\right) .
$$

where the $\left(\boldsymbol{u}_{n}, v_{2 n}, \boldsymbol{\psi}_{n}\right)$, for $n \geq 1$, are the normal modes of $\widetilde{U}$.

The following theorem, which is a copy of Theorem 3.5 with minor modifications, gives the well-posedness result about the system (3.101).

Theorem 3.7. Let $H, A$ and $D(A)$ be defined as above. Then the initial value problem (3.101) is well-posed. That is, for every $U_{0} \in$ $D(A)$, and $F \in L^{1}(0, T ; H)$, with $F^{\prime} \in L^{1}(0, T ; H)$, (3.101) has a unique solution $U$ such that

$$
U \in C([0, T] ; H) \cap L^{\infty}(0, T ; D(A)), \quad \frac{\mathrm{d} U}{\mathrm{~d} t} \in L^{\infty}(0, T ; H) .
$$

Theorem 3.7 is also a direct consequence of Theorem 2.1. The verification of the hypotheses of Theorem 2.1 can be done similarly as in Section 3.3.

For the system of the zero mode (3.96) we can also decompose $\phi_{0}$ into two parts:

$$
\phi_{0}=\bar{\phi}_{0}+\phi_{0}^{\prime}
$$

where $\bar{\phi}_{0}$ is one of the stationary solutions of the equation

$$
\boldsymbol{\sigma}_{\mathbf{4}} \cdot \overline{\boldsymbol{\phi}}_{0}=-\frac{3}{2} L_{3}^{\frac{1}{2}} f \bar{U}_{0}
$$

Then we impose the boundary conditions on the left boundary:

$$
\left\{\begin{array}{l}
\boldsymbol{u}_{0}(0, t)=\boldsymbol{u}^{l}(t), \\
v_{20}(0, t)=v_{2}^{l}(t), \\
\boldsymbol{\phi}_{0}^{\prime}(0, t)=\phi^{\prime l}(t) .
\end{array}\right.
$$

With the boundary conditions above, we can treat the zero mode in a way similar to that in Section 3.3.4. First, by combining the first and second equations (now with $\phi_{0}^{\prime}$ ) of (3.96), we find and then solve the resulting equation for $\boldsymbol{\sigma}_{\boldsymbol{4}} \cdot \boldsymbol{u}_{0}-v_{20 x}$. Once $\boldsymbol{\sigma}_{\boldsymbol{4}} \cdot \boldsymbol{u}_{0}-v_{20 x}$ is known, say $\boldsymbol{\sigma}_{\mathbf{4}} \cdot \boldsymbol{u}_{0}-v_{20 x}=K(x, t)$, we can solve for $\boldsymbol{u}_{0}$ and $v_{20}$ from this expression and the third equation of (3.96). Then when $\boldsymbol{u}_{0}$ and $v_{20}$ are known, 
the first equation in (3.96) gives $\phi_{0}^{\prime}$. We leave it as an exercise for the reader to check the details, and to address the case of nonhomogeneous boundary conditions as in Section 3.3.5.

\section{The Full 3D Linear Case}

4.1. Equations and Preliminary Results. In this section we consider the Primitive Equations in space dimension 3. We focus on the linearized equations since the boundary condition difficulty is already fully present in the linear case (see Section 2 above).

This section is organized as follows: we first recall the PEs and their linearized form. We also recall the normal modes expansion of the unknowns and their decomposition into the subcritical and supercritical modes. These two sets of modes necessitate different treatments and, unlike in Sections 2 and 3 above, the study of the supercritical modes is not straightforward. This Section 4.1 also contains (Section 4.1.3) a study of the associated stationary operator $\mathcal{A}$, a trace theorem adapted to this stationary operator which shows that if $U=(u, v, \psi)$ and $\mathcal{A} U$ are square integrable, then the traces of $v$ and $\psi$ are defined on the whole boundary and the trace of $u$ is defined on part of the boundary (Section 4.1.4); finally Section 4.1 finishes with the study of the zero mode -in the modal decomposition (Section 4.1.5). Section 4.2 is devoted to the study of the subcritical modes for which the stationary problem, partly elliptic and partly hyperbolic, possesses a regularity result. Section 4.3 is devoted to the study of the supercritical modes handled in a different manner; the stationary problem is then fully hyperbolic, and it does not produce any regularity. Finally in Section 4.4 we consider the full linearized Primitive Equations containing both the subcritical and the supercritical modes and we prove our main existence and uniqueness results for homogeneous and nonhomogeneous boundary conditions.

Note that the boundary conditions proposed here for the subcritical modes are different than those studied in Sections 2 and 3 in dimensions 2 and 2.5; this change is of no importance in view of the computational objectives (see Section 2). The related open problem is the determination of all the sets of boundary conditions making the nonviscous primitive equation well-posed. The full nonlinear PEs with boundary conditions similar to those proposed here, will be studied in a separate work.

4.1.1. Equations and Normal Modes Expansion. We now recall the Primitive Equations (PEs) and their normal modes expansion. The 
reader is referred to Section 1 for further details. The nonlinear 3D Primitive Equations read:

$$
\left\{\begin{array}{l}
\frac{\partial \widetilde{\mathbf{v}}}{\partial t}+(\widetilde{\mathbf{v}} \cdot \nabla) \widetilde{\mathbf{v}}+\widetilde{w} \frac{\partial \widetilde{\mathbf{v}}}{\partial z}+k \times \widetilde{\mathbf{v}}+\frac{1}{\rho_{0}} \nabla \widetilde{p}=0 \\
\frac{\partial \widetilde{p}}{\partial z}=-\widetilde{\rho} g \\
\nabla \cdot \widetilde{\mathbf{v}}+\frac{\partial \widetilde{w}}{\partial z}=0, \\
\frac{\partial \widetilde{T}}{\partial t}+(\widetilde{\mathbf{v}} \cdot \nabla) \widetilde{T}+\widetilde{w} \frac{\partial \widetilde{T}}{\partial z}=0, \widetilde{\rho}=\widetilde{\rho}(\widetilde{T}) .
\end{array}\right.
$$

The notations are as follows: $\widetilde{\mathbf{u}}=(\widetilde{u}, \widetilde{v}, \widetilde{w})$ is the velocity of the water, $\widetilde{\mathbf{v}}$ the horizontal velocity, $\widetilde{\rho}$ is the density, $\widetilde{p}$ the pressure, $\widetilde{T}$ the temperature; $\widetilde{\rho}=\widetilde{\rho}(\widetilde{T})$ is the equation of state. In agreement with the Boussinesq approximation, the density $\rho$ is constant everywhere, $\widetilde{\rho}=\rho_{0}$, except in the second equation (4.1). The salinity equation is not present in (4.1), but this would raise little additional difficulty to take into account the salinity $S$. As indicated before, the viscosity is not present in the equations (4.1), this is a crucial point in this study. Equations (4.1) correspond to the $\beta$-plane approximation of the PEs near the latitude $\theta=\theta_{0}$, and $f=f_{0}+\beta y, f_{0}=\Omega \sin \theta_{0}$ where $\Omega$ is the angular velocity of the earth, and $\beta=(d f / d y)$ at $\theta=\theta_{0}$, that is $\beta=f_{0} / a$ at midlatitudes, $\left(\theta_{0}=\pi / 4\right) ; k$ is the unit vector along the south to north poles; $g$ is the gravitational constant. The domain occupied by the water is $\mathcal{M}=\left(0, L_{1}\right) \times\left(0, L_{2}\right) \times\left(-L_{3}, 0\right)$ in the $O x y z$ system of coordinates.

We linearize Equations (4.1) around the simple uniform stratified flow $(4.2)$

$$
\bar{u}=\bar{U}_{0}, \bar{v}=0, \bar{T}=\bar{T}(z), \bar{\rho}=\rho_{0}\left(1-\alpha\left(\bar{T}-T_{0}\right)\right)
$$

where $\bar{U}_{0}>0, \rho_{0}>0$ and $T_{0}>0$ are reference average values of the density and the temperature, $\alpha>0$ is a constant and $\bar{T}$ and $\bar{\rho}$ are linear in z. We introduce the Brunt-Väisälä (buoyancy) frequency

$$
N^{2}=-\frac{g}{\rho_{0}} \frac{d \bar{\rho}}{d z}
$$


and we assume that $N$ does not depend on $z$. We write $\phi=p / \rho_{0}$, $\psi=g T / T_{0}$, and we set $\widetilde{u}=\bar{u}+u, \widetilde{v}=\bar{v}+v$, etc. We obtain as in (1.13):

$$
\left\{\begin{array}{l}
u_{t}+\bar{U}_{0} u_{x}-f v+\phi_{x}=0 \\
v_{t}+\bar{U}_{0} v_{x}+f u+\phi_{y}=0 \\
\psi_{t}+\bar{U}_{0} \psi_{x}+N^{2} w=0 \\
u_{x}+v_{y}+w_{z}=0 \\
\phi_{z}=\psi
\end{array}\right.
$$

4.1.2. Normal modes expansion. As indicated in Section 1, the first step of the analysis of (4.3) consists, by separation of variables, in looking for solutions of the form

$$
\left\{\begin{array}{l}
u(x, y, z, t)=\mathcal{U}(z) \hat{u}(x, y, t), v(x, y, z, t)=\mathcal{V}(z) \hat{v}(x, y, t), \\
\psi(x, y, z, t)=\Psi(z) \hat{\psi}(x, y, t), \\
w(x, y, z, t)=\mathcal{W}(z) \hat{w}(x, y, t), \phi(x, y, z, t)=\Phi(z) \hat{\phi}(x, y, t) .
\end{array}\right.
$$

Substituting these expressions in (4.3), we end up with the following systems (see Section 1 above), for $n \geq 1$,

$$
\left\{\begin{array}{l}
\frac{\partial u_{n}}{\partial t}+\bar{U}_{0} \frac{\partial u_{n}}{\partial x}-f v_{n}+\frac{\partial \phi_{n}}{\partial x}=0 \\
\frac{\partial v_{n}}{\partial t}+\bar{U}_{0} \frac{\partial v_{n}}{\partial x}+f u_{n}+\frac{\partial \phi_{n}}{\partial y}=0 \\
\frac{\partial \psi_{n}}{\partial t}+\bar{U}_{0} \frac{\partial \psi_{n}}{\partial x}+N^{2} w_{n}=0 \\
\phi_{n}=-\frac{1}{\lambda_{n}} \psi_{n}, \quad w_{n}=-\frac{1}{\lambda_{n}}\left(\frac{\partial u_{n}}{\partial x}+\frac{\partial v_{n}}{\partial y}\right)
\end{array}\right.
$$


For $n=0, w_{0}=\psi_{0}=0$ and there remains

$$
\left\{\begin{array}{l}
\frac{\partial u_{0}}{\partial t}+\bar{U}_{0} \frac{\partial u_{0}}{\partial x}-f v_{0}+\frac{\partial \phi_{0}}{\partial x}=0 \\
\frac{\partial v_{0}}{\partial t}+\bar{U}_{0} \frac{\partial v_{0}}{\partial x}+f u_{0}+\frac{\partial \phi_{0}}{\partial y}=0 \\
\frac{\partial u_{0}}{\partial x}+\frac{\partial v_{0}}{\partial y}=0
\end{array}\right.
$$

Note that, since the considered problem is linear, there is no coupling between the different modes; see e.g. Section 2 above for the nonlinear case which indroduces these couplings. We will study the zero mode separately (see Section(4.1.5)), and, for $n \geq 1$, we use the last two equations (4.5) and rewrite the first three in the form

$$
\left\{\begin{array}{c}
\frac{\partial u_{n}}{\partial t}+\bar{U}_{0} \frac{\partial u_{n}}{\partial x}-f v_{n}-\frac{1}{\lambda_{n}} \frac{\partial \psi_{n}}{\partial x}=0 \\
\frac{\partial v_{n}}{\partial t}+\bar{U}_{0} \frac{\partial v_{n}}{\partial x}+f u_{n}-\frac{1}{\lambda_{n}} \frac{\partial \psi_{n}}{\partial y}=0 \\
\frac{\partial \psi_{n}}{\partial t}+\bar{U}_{0} \frac{\partial \psi_{n}}{\partial x}-\frac{N^{2}}{\lambda_{n}}\left(\frac{\partial u_{n}}{\partial x}+\frac{\partial v_{n}}{\partial y}\right)=0 .
\end{array}\right.
$$

As indicated before, our aim is to propose boundary conditions for (4.5)-(4.7) which make these equations well-posed and consequently the equations (4.3) also. As we shall see (see also Sections 2 and 3 above), the boundary conditions are different depending on whether

$$
1 \leq n \leq n_{c}, \quad \text { or } \quad n>n_{c},
$$

where $n_{c}, \lambda_{n_{c}}$ are such that

$$
\frac{n_{c} \pi}{L_{3}}=\lambda_{n_{c}}<\frac{N}{\bar{U}_{0}}<\lambda_{n_{c}+1}=\frac{\left(n_{c}+1\right) \pi}{L_{3}} .
$$

We will not study the nongeneric case where $L_{3} N / \pi \bar{U}_{0}$ is an integer.

The modes $0 \leq n \leq n_{c}$ are called subcritical, and the modes $n>$ $n_{c}$ are called supercritical. It is convenient to introduce the sub and supercritical components of the functions defined by:

$$
u^{0}=P_{0} u=\mathcal{U}_{0} u_{0}, u^{I}=P_{I} u=\sum_{n=1}^{n_{c}} \mathcal{U}_{n} u_{n}, u^{I I}=P_{I I} u=\sum_{n>n_{c}}^{\infty} \mathcal{U}_{n} u_{n}
$$


and similarly for all the other functions; of course the zero mode $u^{0}$ is a subcritical mode, but, as we will see, we need to treat it separately. With these notations, the equations (4.3), (4.5), (4.7) are equivalent to the following systems:

$$
\begin{gathered}
\left\{\begin{array}{c}
u_{t}^{0}+\bar{U}_{0} u_{x}^{0}-f v^{0}+\phi_{x}^{0}=0, \\
v_{t}^{0}+\bar{U}_{0} v_{x}^{0}+f u^{0}+\phi_{y}^{0}=0, \\
u_{x}^{0}+v_{y}^{0}=0,
\end{array}\right. \\
\left\{\begin{array}{c}
u_{t}^{I}+\bar{U}_{0} u_{x}^{I}-f v^{I}+\phi_{x}^{I}=0, \\
v_{t}^{I}+\bar{U}_{0} v_{x}^{I}+f u^{I}+\phi_{y}^{I}=0, \\
\psi_{t}^{I}+\bar{U}_{0} \psi_{x}^{I}+N^{2} w^{I}=0,
\end{array}\right. \\
\left\{\begin{array}{l}
u_{t}^{I I}+\bar{U}_{0} u_{x}^{I I}-f v^{I I}+\phi_{x}^{I I}=0, \\
v_{t}^{I I}+\bar{U}_{0} u_{x}^{I I}+f u^{I I}+\phi_{y}^{I I}=0, \\
\psi_{t}^{I I}+\bar{U}_{0} \psi_{x}^{I I}+N^{2} w^{I I}=0,
\end{array}\right.
\end{gathered}
$$

with the additional relations $\phi=\phi(\psi), w=w(u, v)$ :

$$
\left\{\begin{array}{l}
\phi^{I}=-\sum_{n=1}^{n_{c}} \frac{1}{\lambda_{n}} \psi_{n} \mathcal{U}_{n}, \quad w^{I}=-\sum_{n=1}^{n_{c}} \frac{1}{\lambda_{n}}\left(u_{n x}+v_{n y}\right) \mathcal{W}_{n} \\
\phi^{I I}=-\sum_{n>n_{c}} \frac{1}{\lambda_{n}} \psi_{n} \mathcal{U}_{n}, \quad w^{I I}=-\sum_{n>n_{c}} \frac{1}{\lambda_{n}}\left(u_{n x}+v_{n y}\right) \mathcal{W}_{n}
\end{array}\right.
$$

We will also set $U=(u, v, \psi), U^{0}=P_{0} U, U^{I}=P_{I} U, U^{I I}=P_{I I} U$.

Hereafter, our aim will be to study separately the subscritical and supercritical modes, proposing suitable boundary conditions for them, and to combine them and obtain existence, uniqueness and regularity of the solution $U$. In each case we will study one (subcritical/supercritical) mode separately and then combine them for the whole subcritical and supercritical components. We now conclude this section with some remarks concerning the stationary (time independent) equations associated with (4.6), (4.7), and by a trace theorem which will be used repeatedly in the sequel.

4.1.3. The stationary equations associated with (4.6)-(4.7). The (physical) spatial domain under consideration will be $\mathcal{M}=\mathcal{M}^{\prime} \times\left(-L_{3}, 0\right)$, where $\mathcal{M}^{\prime}$ is the interface atmosphere/ocean, $\mathcal{M}^{\prime}=\left(0, L_{1}\right) \times\left(0, L_{2}\right)$.

We introduce, componentwise, the differential operators $\mathcal{A}_{n}=\left(\mathcal{A}_{n 1}\right.$, $\left.\mathcal{A}_{n 2}, \mathcal{A}_{n 3}\right)$ operating on $U_{n}=\left(u_{n}, v_{n}, \psi_{n}\right)$, 


$$
\mathcal{A}_{n} U_{n}=\left\{\begin{array}{l}
\bar{U}_{0} u_{n x}-\frac{1}{\lambda_{n}} \psi_{n x}, \\
\bar{U}_{0} v_{n x}-\frac{1}{\lambda_{n}} \psi_{n y}, \\
\bar{U}_{0} \psi_{n x}-\frac{N^{2}}{\lambda_{n}}\left(u_{n x}+v_{n y}\right),
\end{array}\right.
$$

with $\bar{U}_{0}, N$ and $\lambda_{n}>0$ as above.

Our object here is to study (recall) the nature of the stationary (time independent) equations in $\mathcal{M}^{\prime}$ :

$$
\mathcal{A}_{n} U_{n}=F_{n}=\left(F_{u n}, F_{v n}, F_{\psi n}\right), \quad n \geq 1 .
$$

We momentarily drop the indices $n$ for the sake of simplicity and although this is not of direct use in the sequel, it is useful to look for the characteristics of the differential system $\mathcal{A} U=F$. We write this system in the matrix form

$$
\mathcal{E} U_{x}+\mathcal{G} U_{y}=F
$$

with

$$
\mathcal{E}=\left(\begin{array}{ccc}
\bar{U}_{0} & 0 & -\frac{1}{\lambda} \\
0 & \bar{U}_{0} & 0 \\
-\frac{N^{2}}{\lambda} & 0 & \bar{U}_{0}
\end{array}\right), \quad \mathcal{G}=\left(\begin{array}{ccc}
0 & 0 & 0 \\
0 & 0 & -\frac{1}{\lambda} \\
0 & -\frac{N^{2}}{\lambda} & 0
\end{array}\right)
$$

and the equation of the characteristics (see e.g. John (1982)) is given by

$$
\operatorname{det}(\mathcal{E} d x-\mathcal{G} d y)=0
$$

that is

$$
\operatorname{det}\left|\begin{array}{ccc}
\bar{U}_{0} \mu & 0 & -\frac{\mu}{\lambda} \\
0 & \bar{U}_{0} \mu & \frac{1}{\lambda} \\
-\frac{N^{2} \mu}{\lambda} & \frac{N^{2}}{\lambda} & \bar{U}_{0} \mu
\end{array}\right|=0
$$

with $\mu=d x / d y$. Hence the equation for $\mu$ :

$$
\bar{U}_{0} \mu\left[\left(\bar{U}_{0}^{2}-\frac{N^{2}}{\lambda^{2}}\right) \mu^{2}-\frac{N^{2}}{\lambda^{2}}\right]=0
$$


The (real) solution $\mu_{0}=0$ exists in all cases, producing the characteristics $x=$ constant (parallel to the background flow $\bar{U}_{0} e_{x}$ ). This corresponds to the first equation:

$$
\frac{\partial}{\partial x}\left(\bar{U}_{0} u-\frac{\psi}{\lambda}\right)=F_{u}+f v .
$$

Then in the supercritical case, $\bar{U}_{0}^{2}-N^{2} \lambda^{-2}>0$ and we have two more real characteristics

$$
\frac{d x_{ \pm}}{d y}=\mu_{ \pm}= \pm \frac{N}{\lambda}\left(\bar{U}_{0}^{2}-\frac{N^{2}}{\lambda^{2}}\right)^{-1 / 2}
$$

whereas, in the subcritical case, these two characteristics are imaginary.

For the stationary zero mode, we obtain from (4.6) after dropping the Coriolis term:

$$
\begin{aligned}
& \bar{U}_{0} u_{x}+\phi_{x}=F_{u}, \\
& \bar{U}_{0} v_{x}+\phi_{y}=F_{v}, \\
& u_{x}+v_{y}=0 .
\end{aligned}
$$

By elimination of $\phi$ we find

$$
\bar{U}_{0}\left(u_{x y}-v_{x x}\right)=F_{u, y}-F_{v, x}
$$

and hence we find the fully elliptic equation

$$
v_{x x}+v_{y y}=\frac{1}{\bar{U}_{0}}\left(F_{v, x}-F_{u, y}\right) .
$$

We infer from this remark that the stationary system $\mathcal{A}_{n} U_{n}=F_{n}$ is fully elliptic for the zero mode, partly hyperbolic and partly elliptic for the other subcritical modes (one real characteristic) and fully hyperbolic in the supercritical case (three real characteristics). This remark will underly the studies in Sections 4.2 and 4.3, although, as we said, we do not use it directly.

4.1.4. A trace theorem. We consider the same differential operator $\mathcal{A}=$ $\left(\mathcal{A}_{1}, \mathcal{A}_{2}, \mathcal{A}_{3}\right)$, as in (4.14) operating on $U=(u, v, \psi)$, but the indices $n$ are dropped for the sake of simplicity: 


$$
\mathcal{A} U=\left\{\begin{array}{l}
\bar{U}_{0} u_{x}-\frac{1}{\lambda} \psi_{x}, \\
\bar{U}_{0} v_{x}-\frac{1}{\lambda} \psi_{y}, \\
\bar{U}_{0} \psi_{x}-\frac{N^{2}}{\lambda}\left(u_{x}+v_{y}\right),
\end{array}\right.
$$

with $\bar{U}_{0}, N, \lambda=\lambda_{n}>0$ as above, and we consider the space ${ }^{16}$

$$
\mathcal{X}=\left\{U \in L^{2}\left(\mathcal{M}^{\prime}\right)^{3}, \mathcal{A} U \in L^{2}\left(\mathcal{M}^{\prime}\right)^{3}\right\},
$$

endowed with its natural Hilbert norm $\left(|U|_{L^{2}\left(\Gamma_{i}\right)^{3}}^{2}+|\mathcal{A} U|_{L^{2}\left(\Gamma_{i}\right)^{3}}^{2}\right)^{\frac{1}{2}}$. We have

Theorem 4.1. If $U=(u, v, \psi) \in \mathcal{X}$, the traces of $v$ and $\psi$ are defined on all of $\partial \mathcal{M}^{\prime}$, the trace of $u$ is defined at $x=0$ and $L_{1}$, and they belong to the respective spaces $H_{x}^{-1}\left(0, L_{1}\right)$ and $H_{y}^{-1}\left(0, L_{2}\right)$. Furthermore the trace operators are linear continuous in the corresponding spaces, e.g. $\left.U \in \mathcal{X} \rightarrow u\right|_{x=0}$ is continuous from $\mathcal{X}$ into $H_{y}^{-1}\left(0, L_{2}\right)$.

Proof. Let us write $\mathcal{A} U=F=\left(f_{1}, f_{2}, f_{3}\right)$. Since $U=(u, v, \psi) \in$ $L^{2}\left(\mathcal{M}^{\prime}\right)^{3}=L_{x}^{2}\left(0, L_{1} ; L_{y}^{2}\left(0, L_{2}\right)^{3}\right)$, we see that $U_{y}=\partial U / \partial y$ belongs to $L_{x}^{2}\left(0, L_{1} ; H_{y}^{-1}\left(0, L_{2}\right)^{3}\right)$. From $\mathcal{A}_{2} U=\bar{U}_{0} v_{x}-\lambda^{-1} \psi_{y}=f_{2} \in L^{2}\left(\mathcal{M}^{\prime}\right)$, we conclude that $v_{x} \in L_{x}^{2}\left(0, L_{1} ; H_{y}^{-1}\left(0, L_{2}\right)\right)$, and $v \in \mathcal{C}\left(\left[0, L_{1}\right] ; H_{y}^{-1}\left(0, L_{2}\right)\right)$, so that its traces at $x=0$ and $L_{1}$ are defined and belong to $H_{y}^{-1}\left(0, L_{2}\right)$. We then have

$$
\begin{aligned}
& \bar{U}_{0} u_{x}-\lambda^{-1} \psi_{x}=f_{1} \in L_{x}^{2}\left(0, L_{1} ; L_{y}^{2}\left(0, L_{2}\right)\right), \\
& \bar{U}_{0} \psi_{x}-\left(N^{2} / \lambda\right) u_{x}=f_{2}-\left(N^{2} / \lambda\right) v_{y} \in L_{x}^{2}\left(0, L_{1} ; H_{y}^{-1}\left(0, L_{2}\right)\right),
\end{aligned}
$$

so that both $u_{x}$ and $\psi_{x}$ belong to the last space and

$$
u, \psi \in \mathcal{C}\left(\left[0, L_{1}\right] ; H_{y}^{-1}\left(0, L_{2}\right)\right) .
$$

Their traces are defined as well at $x=0$ and $L_{1}$. Finally we write

$$
\begin{gathered}
\bar{U}_{0} v_{x}-\lambda^{-1} \psi_{y}=f_{2}, \\
\left(\bar{U}_{0}-N^{2} / \lambda^{2} \bar{U}_{0}\right) \psi_{x}-\left(N^{2} / \lambda\right) v_{y}=f_{3}+N^{2} f_{1} / \lambda \bar{U}_{0},
\end{gathered}
$$

from which we conclude that $v_{y}$ and $\psi_{y} \in L_{y}^{2}\left(0, L_{2} ; H_{x}^{-1}\left(0, L_{1}\right)\right)$ and thus $v$ and $\psi \in \mathcal{C}_{y}\left(\left[0, L_{2}\right] ; H_{x}^{-1}\left(0, L_{1}\right)\right)$ and their traces are both defined

\footnotetext{
${ }^{16}$ We will write $\mathcal{A}_{n}, \mathcal{X}_{n}$ when it is necessary to emphasize the dependence on $n$ through $\lambda\left(\lambda=\lambda_{n}\right)$.
} 
at $y=0$ and $L_{2}$. Finally all the mappings above are continuous, and the theorem is proved.

Remark 4.2. Although the values of $\bar{U}_{0}, N, \lambda=\lambda_{n}$ are intended to be those above, Theorem 4.1 extends to operators $\mathcal{A}$ with the same structure and more general constant coefficients, and it will be used in this way at times.

4.1.5. The zero mode. The equations for this mode appear in (4.6) but, for the convenience of the notations, the subscripts are now changed to superscripts. Due to the form of the third equation, we proceed by analogy with the incompressible Navier Stokes equations and we determine first $\mathbf{u}^{0}=\left(u^{0}, v^{0}\right)$ and then $\phi^{0}$ by solving a Neumann problem. The natural function space for $\mathbf{u}^{0}$ is

$$
H^{0}=\left\{\mathbf{u}^{0}=\left(u^{0}, v^{0}\right) \in L^{2}\left(\mathcal{M}^{\prime}\right)^{2}, u_{x}^{0}+v_{y}^{0}=0, \mathbf{u}^{0} \cdot \mathbf{n}=0 \text { on } \partial \mathcal{M}^{\prime}\right\},
$$

where $\mathbf{n}=\left(n_{x}, n_{y}\right)$ is the unit outward normal on $\partial \mathcal{M}^{\prime}$. Recall (see e.g. Temam (2001)) that the trace of $\mathbf{u}^{0} \cdot \mathbf{n}$ on $\partial \mathcal{M}^{\prime}$ makes sense for $\mathbf{u}^{0} \in L^{2}\left(\mathcal{M}^{\prime}\right)^{2}$ with $\operatorname{div} \mathbf{u}^{0}=u_{x}^{0}+v_{y}^{0} \in L^{2}\left(\mathcal{M}^{\prime}\right)$. If the test function $\widetilde{\mathbf{u}}^{0}=\left(\widetilde{u}^{0}, \widetilde{v}^{0}\right) \in H^{0}$ is smooth, we classically see that (4.6) implies that

$$
\frac{d}{d t}\left(\mathbf{u}^{0}, \widetilde{\mathbf{u}}^{0}\right)_{H^{0}}+\bar{U}_{0}\left(\mathbf{u}_{x}^{0}, \widetilde{\mathbf{u}}^{0}\right)+f\left(e_{z} \wedge \mathbf{u}^{0}, \widetilde{\mathbf{u}}^{0}\right)=0,
$$

where $e_{z}=(0,0,1)$. Conversely if there exists $u^{0}$ such that (4.24) is satisfied for all such $\widetilde{\mathbf{u}}^{0}$, then there exists $\phi^{0}$ such that equations (4.6) are satisfied.

We then introduce the linear unbounded operator $A^{0}$ in $H$,

$$
A^{0} \mathbf{u}^{0}=P_{H^{0}}\left(\bar{U}_{0} \frac{\partial \mathbf{u}^{0}}{\partial x}+f e_{z} \wedge \mathbf{u}^{0}\right)
$$

with domain

$$
D\left(A^{0}\right)=\left\{\mathbf{u}^{0} \in H^{0}, \mathbf{u}_{x}^{0} \in L^{2}\left(\mathcal{M}^{\prime}\right)^{2}\right\},
$$

where $P_{H^{0}}$ is the orthogonal projector in $L^{2}\left(\mathcal{M}^{\prime}\right)^{2}$ onto $H^{0}$. Equation (4.24) is then equivalent to the evolution equation

$$
\frac{d \mathbf{u}^{0}}{d t}+A^{0} \mathbf{u}^{0}=0
$$

Using the Hille-Phillips-Yoshida theorem, it is easy to see that equation (4.27) with initial condition $\mathbf{u}^{0}(0)$ given in $H^{0}$ or $D\left(A^{0}\right)$ produces a wellposed initial value problem. For that purpose it is sufficient to show that $-A^{0}$ is the infinitesimal generator of a contraction semi-group in $H^{0}$. Since the operator $\mathbf{u}^{0} \longrightarrow P_{H^{0}}\left(f e_{z} \wedge \mathbf{u}^{0}\right)$ is continuous in $H^{0}$, it 
suffices to show that $\bar{A}^{0} \mathbf{u}^{0}=P_{H^{0}} \bar{U}^{0} \mathbf{u}_{0 x}$ with domain $D\left(\bar{A}^{0}\right)$ is dense in $H^{0}$ and $\bar{A}^{0}$ is closed which is easy; also $\bar{A}^{0} \geq 0$ as

$$
\begin{aligned}
\left(\bar{A}^{0} \mathbf{u}^{0}, \mathbf{u}^{0}\right)_{H^{0}} & =\bar{U}_{0} \int_{\mathcal{M}^{\prime}} \mathbf{u}_{x}^{0} \cdot \mathbf{u}^{0} d \mathcal{M}^{\prime} \\
& =\frac{\bar{U}_{0}}{2} \int_{0}^{L_{2}}\left[\left|\mathbf{u}^{0}\right|^{2}\left(L_{1}, y\right)-\left|\mathbf{u}^{0}\right|^{2}(0, y)\right] d y \\
& =0
\end{aligned}
$$

the integration in $x$ being justified for $\mathbf{u}^{0} \in D\left(A^{0}\right)$. We need also to show that $\bar{A}^{0 *}$ is positive, but this results from the fact that $\bar{A}^{0 *}=-\bar{A}^{0}$, with the same domain. ${ }^{17}$ We refrain from giving all the details of the proof for this partial result and refer the reader to Section 4.4 for the complete analysis.

4.2. Subcritical Modes. We now proceed and study the subcritical modes $1 \leq n \leq n_{c}$.

4.2.1. One subcritical mode $\left(1 \leq n \leq n_{c}\right)$. We temporarily drop the indices $n$ and first want to set and study an initial value problem for (4.7) when the mode is subcritical, that is (see (4.8))

$$
\lambda=\lambda_{n}<\frac{N}{\bar{U}_{0}} \quad\left(0 \leq n \leq n_{c}\right)
$$

There are several possible choices of suitable boundary conditions; see e.g. different ones in Rousseau, Temam, and Tribbia (2005b) for a related situation. Here, for a simple subcritical mode $1 \leq n \leq n_{c}$, we choose the following boundary conditions:

$$
\left\{\begin{array}{l}
\psi=0 \text { at } x=L_{1}, \quad \text { and } y=0, L_{2}, \\
v=0 \text { and } u=\psi / \lambda \bar{U}_{0} \text { at } x=0,
\end{array}\right.
$$

and we introduce the space

$$
D(A)=\left\{U \in L^{2}\left(\mathcal{M}^{\prime}\right)^{3}, \mathcal{A} U \in L^{2}\left(\mathcal{M}^{\prime}\right)^{3}, U \text { satisfies }(4.29)\right\},
$$

and the operator ${ }^{18}$

$$
A U=\mathcal{A} U
$$

In view of Theorem 4.1, the traces appearing in (4.29) are well defined when $U \in L^{2}\left(\mathcal{M}^{\prime}\right)^{3}$ and $\mathcal{A} U \in L^{2}\left(\mathcal{M}^{\prime}\right)^{3}$, so that the definition of $D\left(A_{n}\right)$ in (4.30) makes sense.

\footnotetext{
${ }^{17}$ Note that $A^{0 *}=-A^{0}$ as well, and of course $D\left(A^{0 *}\right)=D\left(A^{0}\right)=D\left(\bar{A}^{0}\right)$.

${ }^{18}$ When needed we will write also $\mathcal{A}_{n}, A_{n}, D\left(A_{n}\right)$ to emphasize the dependence on $n\left(\lambda=\lambda_{n}\right)$.
} 
Remark 4.3. As indicated above the boundary conditions (4.29) are different than those in Section 2 for dimension two (after neglecting the dependence on $y$ ).

We proceed with a regularity result for $U$ in $D(A)$ (see the comments in Section 4.1.3).

Theorem 4.4. If $U=(u, v, \psi) \in D(A)$, then $v$ and $\psi$ belong to $H^{1}\left(\mathcal{M}^{\prime}\right)$ and $u_{x}$ belongs to $L^{2}\left(\mathcal{M}^{\prime}\right)$

Proof. For $U \in D(A)$, we set $A U=F=\left(f_{1}, f_{2}, f_{3}\right)$. Then, in the distribution sense,

$$
\left\{\begin{array}{l}
\bar{U}_{0} u_{x}-\frac{1}{\lambda} \psi_{x}=f_{1}, \\
\bar{U}_{0} v_{x}-\frac{1}{\lambda} \psi_{y}=f_{2}, \\
\bar{U}_{0} \psi_{x}-\frac{N^{2}}{\lambda}\left(u_{x}+v_{y}\right)=f_{3} .
\end{array}\right.
$$

Combining the first and third equations we find

$$
\bar{U}_{0}\left(1-\frac{N^{2}}{\lambda^{2} \bar{U}_{0}^{2}}\right) \psi_{x}-\frac{N^{2}}{\lambda} v_{y}=\frac{N^{2}}{\lambda \bar{U}_{0}} f_{1}+f_{3} .
$$

Combining this equation with the second equation (4.31), we obtain

$$
\begin{aligned}
& \left(\bar{U}_{0}^{2}-\frac{N^{2}}{\lambda^{2}}\right) \psi_{x x}-\frac{N^{2}}{\lambda^{2}} \psi_{y y}= \\
& =\frac{N^{2}}{\lambda} f_{1 x}+\frac{N^{2}}{\lambda} f_{2 y}+\bar{U}_{0} f_{3 x} .
\end{aligned}
$$

Note that this equation is elliptic in the subcritical case; of course a similar elliptic equation can be derived for $v$, but we will not use it. We associate to this equation the boundary condition $\psi=0$ at $y=0, L_{2}$ and $x=L_{1}$ contained in (4.29). Then for the side $x=0$ of $\mathcal{M}^{\prime}$, a suitable boundary condition is given by (4.32) in which $v_{y}=0$ since $v=0$ at $x=0$; hence

$$
-\frac{\partial \psi}{\partial n}=\psi_{x}=\bar{U}_{0}^{-1}\left(1-\frac{N^{2}}{\lambda^{2} \bar{U}_{0}^{2}}\right)^{-1}\left(\frac{N^{2}}{\lambda \bar{U}_{0}} f_{1}+f_{2}\right), \text { at } x=0 .
$$

The right-hand side of (4.34) does not make sense on $x=0$ for $F \in$ $L^{2}\left(\mathcal{M}^{\prime}\right)^{3}$. So we proceed as follows: we approximate $F$ in $L^{2}\left(\mathcal{M}^{\prime}\right)^{3}$ by 
a sequence of smooth functions $F_{m} \in \mathcal{C}^{\infty}\left(\mathcal{M}^{\prime}\right)^{3}$. For each $m$, the righthand side of (4.34) makes sense and we find a unique solution $\psi_{m}$ of (4.32), (4.34) and $\psi_{m}=0$ on the other sides of $\mathcal{M}^{\prime}$. Of course $\psi_{m}$ is $\mathcal{C}^{\infty}$ on $\mathcal{M}^{\prime}$ away from the corners and $\psi_{m} \in H^{1}\left(\mathcal{M}^{\prime}\right)$ (at least), see Grisvard (1985). Then from $\psi_{m}$, we determine the corresponding $v=v_{m}$ up to an additive constant: $v_{m x}$ and $v_{m y}$ are given by (4.32) and the second equation (4.31), and these equations are compatible (i.e. $v_{m x y}=v_{m y x}$ ), because of (4.33). Note that $v_{m}$ belongs to $H^{1}\left(\mathcal{M}^{\prime}\right)$ at least, its trace on the side $x=0$ of $\mathcal{M}^{\prime}$ is defined, $v_{m y}=0$ on this side because of (4.34). Hence $v_{m}=0$ on $x=0$ by choosing properly the constant. Finally $u_{m}$ is determined by the first equation (4.31) and the boundary condition $U_{m}=\psi_{m} / \lambda \bar{U}_{0}$ at $x=0$. In conclusion $U_{m}=\left(u_{m}, v_{m}, \psi_{m}\right)$ that we just constructed belongs to $D(A)$ and satisfies $A U_{m}=F_{m}$.

To pass to the limit $m \rightarrow \infty$, we obtain the suitable a priori estimates as follows: we multiply the second equation (4.31) by $-\left(N^{2} / \lambda\right) \psi_{m y}$, equation (4.32) by $-\bar{U}_{0} \psi_{m x}$, integrate over $\mathcal{M}^{\prime}$ and add these equations. We find

$$
\begin{aligned}
& \bar{U}_{0}^{2}\left(\frac{N^{2}}{\lambda^{2} \bar{U}_{0}^{2}}-1\right) \int_{\Gamma_{i}} \psi_{m x}^{2} d \mathcal{M}^{\prime}+\frac{N^{2}}{\lambda^{2}} \int_{\mathcal{M}^{\prime}} \psi_{m y}^{2} d \mathcal{M}^{\prime} \\
& +\frac{\bar{U}_{0} N^{2}}{\lambda} \int_{\Gamma i}\left(v_{m y} \psi_{m x}-v_{m x} \psi_{m y}\right) d \mathcal{M}^{\prime}= \\
& =-\int_{\mathcal{M}^{\prime}}\left(\frac{N^{2}}{\lambda} f_{m 2} \psi_{m y}+\frac{N^{2}}{\lambda} f_{m 1} \psi_{m x}+\bar{U}_{0} f_{m 2} \psi_{m x}\right) d \mathcal{M}^{\prime} .
\end{aligned}
$$

The integrals involving $v \psi$ cancel each other because it is legitimate to integrate by parts (enough regularity) and, by integration by parts, taking into account the boundary conditions (4.29) for $U_{m}$, we find

$$
\int_{\mathcal{M}^{\prime}} v_{m y} \psi_{m x} d \mathcal{M}^{\prime}=\int_{\Gamma_{i}} v_{m x} \psi_{m y} d \mathcal{M}^{\prime}
$$

Since $N^{2}>\lambda^{2} \bar{U}_{0}^{2}$, we then easily infer from (4.35) that

$$
\left|\nabla \psi_{m}\right|_{L^{2}\left(\mathcal{M}^{\prime}\right)^{2}} \leq c\left|F_{m}\right|_{L^{2}\left(\Gamma_{i}\right)^{2}} \leq \text { const. }
$$

Thanks to the boundary conditions on $\psi_{m}$ we have a Poincaré inequality which guarantees that

$$
\left|\psi_{m}\right|_{L^{2}\left(\Gamma_{i}\right)} \leq \mathrm{const}
$$

and $\psi_{m}$ is bounded in $H^{1}\left(\mathcal{M}^{\prime}\right)$. As for the construction of $v_{m}$, the second equation (4.31), (4.32) and $v_{m}=0$ on $x=0$ then show that $v_{m}$ is bounded in $H^{1}\left(\mathcal{M}^{\prime}\right)$. Finally $\bar{U}_{0} u_{m}-\psi_{m} / \lambda$ and its $x$ derivative are bounded in $L^{2}\left(\mathcal{M}^{\prime}\right)$ so that $u_{m}$ and $u_{m x}$ are bounded in $L^{2}\left(\mathcal{M}^{\prime}\right)$ as well. 
Passing to the limit $m \rightarrow \infty$, we obtain $U_{m} \rightarrow \bar{U}$, with $\bar{U} \in D(A)$ and $A \bar{U}=F, \bar{U}$ satisfying the desired regularity properties. To conclude, we need to show that $\bar{U}=U$, that is $A$ is one-to-one.

We thus consider $U \in D(A)$, such that $A U=0$. Then $U$ satisfies (4.31) with $f_{1}=f_{2}=f_{3}=0$ and the boundary conditions (4.29): $\psi, v$ also satisfy (4.32), (4.33) and (4.34) with $F=0$. The mixed NeumannDirichlet problem of which $\psi$ is solution shows that $\psi=0$; then $v=0$ because of (4.32), the second equation (4.31) and $v=0$ at $x=0$. Finally $u=0$ because of the first equation (4.31) and the boundary condition $\bar{U}_{0} u-\psi \lambda=0$ at $x=0$.

Theorem 4.4 is thus proved.

4.2.2. Positivity of $A$ and $A^{*}$. We endow the space $H=L^{2}\left(\mathcal{M}^{\prime}\right)^{3}$ with the Hilbert scalar product and norm

$$
(U, \widetilde{U})_{H}=\int_{\Gamma_{i}}\left(u \widetilde{u}+v \widetilde{v}+\frac{1}{N^{2}} \psi \widetilde{\psi}\right) d \mathcal{M}^{\prime}, \quad|U|_{H}=\left\{(U, U)_{H}\right\}^{1 / 2} .
$$

Our aim is now to prove that $A$ and its adjoint $A^{*}$ defined below are positive in the sense

$$
\left\{\begin{array}{l}
(A U, U)_{H} \geq 0, \quad \forall U \in D(A) \\
\left(A^{*} U, U\right)_{H} \geq 0, \quad \forall U \in D\left(A^{*}\right) .
\end{array}\right.
$$

These properties are needed to apply the Hille-Phillips-Yoshida theorem (see Section 4.4). The result for $U$ is now easy thanks to Theorem 4.4. Indeed the following easy calculations are now legitimate, $\forall U \in D(A):$

$$
\begin{aligned}
(A U, U)_{H}= & \int_{\Gamma_{i}}\left[\left(\bar{U}_{0} u_{x}-\frac{1}{\lambda} \psi_{x}\right) u+\left(\bar{U}_{0} v_{x}-\frac{1}{\lambda} \psi_{y}\right) v\right. \\
& \left.+\frac{\bar{U}_{0}}{N^{2}} \psi_{x} \psi-\frac{1}{\lambda}\left(u_{x}+v_{y}\right) \psi\right] d \mathcal{M}^{\prime} \\
= & \frac{\bar{U}_{0}}{2} \int_{0}^{L_{2}}\left(u^{2}+v^{2}\right)\left(L_{1}, y\right) d y \\
& -\int_{0}^{L_{2}}\left[\frac{\bar{U}_{0}}{2}\left(u^{2}+\frac{1}{N^{2}} \psi^{2}\right)(0, y)-\frac{1}{\lambda}(u \psi)(0, y)\right] d y \\
\geq & \frac{\bar{U}_{0}}{2} \int_{0}^{L_{2}}\left(\left(\lambda \bar{U}_{0}\right)^{-2}-N^{-2}\right) \psi^{2}(0, y) d y \geq 0 .
\end{aligned}
$$

All the integrations by parts above are easy to justify for functions in $H^{1}\left(\mathcal{M}^{\prime}\right)$. We just want to emphasize those involving $u$. If $u$ and $\widetilde{u}$ 
belong to $L_{y}^{2}\left(0, L_{2} ; H_{x}^{1}\left(0, L_{1}\right)\right)$, then $u, \widetilde{u} \in L_{y}^{2}\left(0, u_{2} ; \mathcal{C}_{x}\left(\left[0, L_{1}\right]\right)\right)$ and for a.e. $y \in\left(0, L_{2}\right)$ :

$$
\int_{0}^{L_{1}}\left(u_{x} \widetilde{u}+u \widetilde{u}_{x}\right)(x, y) d x=(u \widetilde{u})(1, y)-(u \widetilde{u})(0, y)
$$

and, integrating in $y$,

$$
\begin{aligned}
& \int_{\Gamma_{i}}\left(u_{x} \widetilde{u}+u \widetilde{u}_{x}\right) d x d y= \\
& =\int_{0}^{L_{2}}[(u, \widetilde{u})(1, y)-(u \widetilde{u})(0, y)] d y .
\end{aligned}
$$

To prove (4.40), we apply (4.41) with $\widetilde{u}=u, \psi$, and $v$.

We now turn to the definition of the formal adjoint $A^{*}$ of $A$ and its domain $D\left(A^{*}\right)$, in the sense of the adjoint of a linear unbounded operator (see Rudin (1991)). For that purpose we first compute $(A U, \widetilde{U})_{H}$ for $U$ and $\widetilde{U}$ smooth. By integration by parts, using Stokes formula, we find:

$$
\begin{aligned}
(A U, \widetilde{U})_{H}= & \int_{\Gamma_{i}}\left[\left(\bar{U}_{0} u_{x}-\frac{1}{\lambda} \psi_{x}\right) \widetilde{u}+\left(\bar{U}_{0} v_{x}-\frac{1}{\lambda} \psi_{y}\right) \widetilde{v}\right. \\
& \left.+\frac{\bar{U}_{0}}{N^{2}} \psi_{x} \widetilde{\psi}-\frac{1}{\lambda}\left(u_{x}+v_{y}\right) \widetilde{\psi}\right] d \mathcal{M}^{\prime} \\
= & I_{0}+I_{1},
\end{aligned}
$$

where $I_{0}$ stands for the integrals in $\mathcal{M}^{\prime}$ and $I_{1}$ for the integrals on $\partial \mathcal{M}^{\prime}$. For $I_{0}$ we have

$$
I_{0}=\int_{\Gamma_{i}}\left(\mathcal{A}_{1}^{*} \widetilde{U} u+\mathcal{A}_{2}^{*} \widetilde{U} v+N^{-2} \mathcal{A}_{3}^{*} \widetilde{U} \psi\right) d \mathcal{M}^{\prime},
$$

with $\mathcal{A}^{*} \widetilde{U}=\left(\mathcal{A}_{1}^{*} \widetilde{U}, \mathcal{A}_{2}^{*}, \widetilde{U}, \mathcal{A}_{3}^{*} \widetilde{U}\right)$, and

$$
\mathcal{A}^{*} \widetilde{U}=\left\{\begin{array}{l}
-\bar{U}_{0} \widetilde{u}_{x}+\frac{1}{\lambda} \widetilde{\psi}_{x}, \\
-\bar{U}_{0} \widetilde{v}_{x}+\frac{1}{\lambda} \widetilde{\psi}_{y}, \\
-\bar{U}_{0} \widetilde{\psi}_{x}+\frac{N^{2}}{\lambda}\left(\widetilde{u}_{x}+\widetilde{v}_{y}\right) .
\end{array}\right.
$$


For $I_{1}$, taking into account the boundary conditions (4.29), there remains:

$$
\begin{aligned}
I_{1} & =\int_{0}^{L_{2}}\left[\bar{U}_{0}(u \widetilde{u})\left(L_{1}, y\right)+\bar{U}_{0}(v \widetilde{v})\left(L_{1}, y\right)-\frac{1}{\lambda}(u \widetilde{\psi})\left(L_{1}, y\right)\right] d y \\
& +\int_{0}^{L_{2}}\left(-\frac{\bar{U}_{0}}{N^{2}}+\frac{1}{\lambda^{2} \bar{U}_{0}}\right)(\psi \widetilde{\psi})(0, y) d y \\
& -\lambda^{-1} \int_{0}^{L_{1}}\left[(v \widetilde{\psi})\left(x, L_{2}\right)+(v \widetilde{\psi})(x, 0)\right] d x .
\end{aligned}
$$

According to Rudin (1991), $D\left(A^{*}\right)$ consists of the $\widetilde{U}$ in $H$ such that $U \rightarrow(A U, \widetilde{U})_{H}$ is continuous on $D(A)$ for the topology (norm) of $H$. If $U$ is restricted to the class of $\mathcal{C}^{\infty}$ functions with compact support in $\mathcal{M}^{\prime}$ (endowed with the norm of $H$ ), then $I_{1}=0$, and $U \rightarrow I_{0}$ can only be continuous if $\mathcal{A}^{*} \widetilde{U}$ as defined in (4.44) belongs to $L^{2}\left(\mathcal{M}^{\prime}\right)^{3}$. We then observe that Theorem 4.1 applies to $\mathcal{A}^{*}$ as well and to more general constant coefficients operators. Hence if $\widetilde{U} \in D\left(A^{*}\right)$ then $\widetilde{U} \in L^{2}\left(\mathcal{M}^{\prime}\right)^{3}$ with $\mathcal{A}^{*} \widetilde{U} \in L^{2}\left(\mathcal{M}^{\prime}\right)^{3}$, and the traces of $\widetilde{U}$ are defined as in Theorem 4.1. We now restrict $U$ to the class of $\mathcal{C}^{\infty}$ functions on $\overline{\mathcal{M}}^{\prime}$ which belong to $D(A)$. Then the expressions above of $I_{0}$ and $I_{1}$ show that $U \rightarrow(A U, \widetilde{U})_{H}$ can only be continuous in $U$ for the topology (norm) of $H$ if the following boundary conditions are satisfied:

$$
\left\{\begin{array}{l}
\widetilde{\psi}=0 \text { at } y=0, L_{2} \text { and } x=0, \\
\widetilde{v}=0 \text { and } \widetilde{u}=\widetilde{\psi} / \lambda \bar{U}_{0} \text { at } x=L_{1} .
\end{array}\right.
$$

Hence we conclude that ${ }^{19}$

$$
D\left(A^{*}\right)=\left\{\widetilde{U} \in L^{2}\left(\mathcal{M}^{\prime}\right)^{3}, \mathcal{A}^{*} \widetilde{U} \in L^{2}\left(\mathcal{M}^{\prime}\right)^{3} \text {, and } \widetilde{U} \text { satisfies (4.45) }\right\} .
$$

We have shown indeed that $D\left(A^{*}\right)$ is included in the right-hand side of (4.46). Now, with exactly the same reasoning as in Theorem 4.4, we can show that if $\widetilde{U}=(\widetilde{u}, \widetilde{v}, \widetilde{\psi}) \in D\left(A^{*}\right)$, then $\widetilde{v}$ and $\widetilde{\psi}$ belong to $H^{1}\left(\mathcal{M}^{\prime}\right)$ and $\widetilde{u}_{x}$ belongs to $L^{2}\left(\mathcal{M}^{\prime}\right)$.

Thus using again (4.41), we see that for every $U$ in $D(A)$ and $\widetilde{U}$ in $D\left(A^{*}\right)$ (not necessarily $\mathcal{C}^{\infty}$ ), then $(A U, \widetilde{U})_{H}=I_{0}+I_{1}$ as above, with $I_{1}=0$ and $I_{0}$ as in $(4.43)$, so that $U \rightarrow(A U, \widetilde{U})_{H}$ is continuous on $D(A)$ for the norm of $H$. The opposite inclusion is proven and

\footnotetext{
${ }^{19}$ Similarly we write $\mathcal{A}_{n}^{*}, A_{n}^{*}, D\left(A_{n}^{*}\right)$ when the dependence on $n$ needs to be emphasized $\left(\lambda=\lambda_{n}\right)$.
} 
(4.46) is established. This reasoning also shows that, for every $\widetilde{U} \in$ $D\left(A^{*}\right), A^{*} \widetilde{U}=\mathcal{A}^{*} \widetilde{U}, \mathcal{A}^{*}$ as in (4.44).

It is now easy to prove the positivity of $A^{*}$, that is the second statement in (4.39). We proceed as in (4.40), using (4.41):

$$
\begin{aligned}
& \left(A^{*} U, U\right)_{H}=\int_{\Gamma_{i}}\left[\left(-\bar{U}_{0} u_{x}+\frac{1}{\lambda} \psi_{x}\right) u+\right. \\
& \left.\quad\left(-\bar{U}_{0} v_{x}+\frac{1}{\lambda} \psi_{y}\right) v+\left(-\frac{\bar{U}_{0}}{N^{2}} \psi_{x}+\frac{1}{\lambda}\left(u_{x}+v_{y}\right)\right) \psi\right] d \mathcal{M}^{\prime} \\
& =\text { (using the boundary conditions }(4.45)) \\
& =\frac{\bar{U}_{0}}{2} \int_{0}^{L_{2}}\left(u^{2}+v^{2}\right)(0, y) d y \\
& -\int_{0}^{L_{2}}\left[\frac{\bar{U}_{0}}{2}\left(u^{2}+\frac{1}{N^{2}} \psi^{2}\right)\left(L_{1}, y\right)-\frac{1}{\lambda}(u \psi)\left(L_{1}, y\right)\right] d y \\
& \geq \frac{\bar{U}_{0}}{2} \int_{0}^{L_{2}}\left(\left(\lambda \bar{U}_{0}\right)^{-2}-N^{-2}\right) \psi^{2}\left(L_{1}, y\right) d y \geq 0 .
\end{aligned}
$$

Hence the positivity. Note that we cannot just write $\left(A^{*} U, U\right)_{H}=$ $(A U, U)_{H} \geq 0$, because $U$ in $D\left(A^{*}\right)$ may not belong to $D(A)$. In summary we have proven the following theorem:

Theorem 4.5. For every $U \in D\left(A_{n}\right)$, as defined in (4.30), we have $\left(A_{n} U, U\right)_{L^{2}\left(\mathcal{M}^{\prime}\right)^{3}} \geq 0$. Similarly, for every $U \in D\left(A_{n}^{*}\right)$ defined in (4.46), we have $\left(A_{n}^{*} U, U\right)_{L^{2}\left(\mathcal{M}^{\prime}\right)^{3}} \geq 0$.

Remark 4.6. Based on the previous results we can show that, for each $n, 1 \leq n \leq n_{c},-A=-A_{n}$ is the infinitesimal generator of a contraction semi-group. Then by application of the Hille-Yoshida theorem we can solve the initial and boundary value problem associated with equations (4.7) for every such $n$. We refrain from developing this and will instead establish a well-posedness result for all modes together, see Section 4.4.

4.3. Supercritical Modes. We now consider the initial and boundary value problem for one single supercritical mode, that is equations (4.5) or equivalently (4.7) when $n>n_{c}$. We temporarily drop the indices $n$, and write e.g.

$$
\lambda=\lambda_{n}>\frac{N}{\bar{U}_{0}}
$$


4.3.1. The operator $A$ and its adjoint $A^{*}$. Here, for one supercritical mode we choose the following boundary conditions:

$$
\left\{\begin{array}{l}
u, v, \text { and } \psi=0 \text { at } x=0, \\
\text { and } \psi=0 \text { at } y=0 \text { and } L_{2} .
\end{array}\right.
$$

In this case the operator $A=A_{n}$, is defined by $A U=\mathcal{A} U$ as in (4.21), and

$$
D(A)=\left\{U \in H=L^{2}\left(\mathcal{M}^{\prime}\right)^{2}, \mathcal{A} U \in L^{2}\left(\mathcal{M}^{\prime}\right), U \text { satisfies }(4.48)\right\} .
$$

Note that, according to Theorem 4.1, the traces of $u, v, \psi$ appearing in (4.48) and (4.49) are well-defined when $U \in L^{2}\left(\mathcal{M}^{\prime}\right)^{3}$ and $\mathcal{A} U \in$ $L^{2}\left(\mathcal{M}^{\prime}\right)^{3}$.

In view of proving that $-A=-A_{n}$ is the infinitesimal generator of a contraction semigroup, our main task is now to show that

$$
\left\{\begin{array}{l}
(A U, U)_{H} \geq 0, \forall U \in D(A), \text { and } \\
\left(A^{*} U, U\right)_{H} \geq 0, \forall U \in D\left(A^{*}\right)^{20}
\end{array}\right.
$$

where $A^{*}$ is defined below. Our approach for (4.50) is however different from the subcritical case which was based on the regularity result Theorem 4.4. In the supercritical case the equations are hyperbolic and there are no similar regularity results. Instead we are going to prove that $(A U, U)_{H} \geq 0$ when $U$ is sufficiently regular; then we define $A^{*}$ and prove that $\left(A^{*} U, U\right)_{H} \geq 0$ for every $U$, sufficiently regular, in the domain of $A^{*}$; and finally, by passage to the limit, we prove (4.50) for all functions in $D(A)$ and $D\left(A^{*}\right)$ respectively.

Positivity of $A$ 
We prove that $(A U, U)_{H} \geq 0$ when $U$ belongs to $D(A)$ and is sufficiently regular (say in $\left.\mathcal{C}^{2}\left(\overline{\mathcal{M}}^{\prime}\right)^{3}\right)$ :

$$
\begin{aligned}
(A U, U)_{H}= & \int_{\mathcal{M}^{\prime}}\left[\left(\bar{U}_{0} u_{x}-\frac{1}{\lambda} \psi_{x}\right) u+\left(\bar{U}_{0} v_{x}-\frac{1}{\lambda} \psi_{y}\right) v\right. \\
& \left.+\frac{1}{N^{2}}\left(\bar{U}_{0} \psi_{x}-\frac{N^{2}}{\lambda}\left(u_{x}+v_{y}\right)\right) \psi\right] d \mathcal{M}^{\prime} \\
= & (\operatorname{using}(4.48) \text { and }(4.47)) \\
= & \frac{\bar{U}_{0}}{2} \int_{0}^{L_{2}}\left(u^{2}+v^{2}+\frac{1}{N^{2}} \psi^{2}\right)\left(L_{1}, y\right) d y \\
& -\frac{1}{\lambda} \int_{0}^{L_{2}}(u \psi)\left(L_{1}, y\right) d y \\
= & \frac{\bar{U}_{0}}{2} \int_{0}^{L_{2}} v^{2}\left(L_{1}, y\right) d y+ \\
& +\frac{\bar{U}_{0}}{2} \int_{0}^{L_{2}}\left(u^{2}+\frac{1}{N^{2}} \psi^{2}-\frac{2}{\bar{U}_{0}} u \psi\right)\left(L_{1}, y\right) d y \\
\geq & 0 .
\end{aligned}
$$

The adjoint $A^{*}$

Assume that $U \in D(A)$ and $\widetilde{U} \in H$ are smooth functions; then, as in (4.42):

$$
\begin{aligned}
(A U, \widetilde{U})_{H}= & \int_{\mathcal{M}^{\prime}}\left[\left(\bar{U}_{0} u_{x}-\frac{1}{\lambda} \psi_{x}\right) \widetilde{u}+\left(\bar{U}_{0} v_{x}-\frac{1}{\lambda} \psi_{y}\right) \widetilde{v}\right. \\
& \left.+\frac{1}{N^{2}}\left(\bar{U}_{0} \psi_{x}-\frac{N^{2}}{\lambda}\left(u_{x}+v_{y}\right)\right) \widetilde{\psi}\right] d \mathcal{M}^{\prime} \\
= & I_{0}+I_{1},
\end{aligned}
$$

where $I_{0}$ stands for the integrals on $\mathcal{M}^{\prime}$ and $I_{1}$ for the integrals on $\partial \mathcal{M}^{\prime}$. For $I_{0}$, we have

$$
I_{0}=\int_{\mathcal{M}^{\prime}}\left(\mathcal{A}_{1}^{*} \widetilde{U} u+\mathcal{A}_{2}^{*} \widetilde{U} v+N^{-2} \mathcal{A}_{3}^{*} \widetilde{U} \psi\right) d \mathcal{M}^{\prime}
$$


with $\mathcal{A}^{*} \widetilde{U}=\left(\mathcal{A}_{1}^{*} \widetilde{U}, \mathcal{A}_{2}^{*} \widetilde{U}, \mathcal{A}_{3}^{*} \widetilde{U}\right)$ as in (4.44). For $I_{1}$, taking into account the boundary conditions (4.48), there remains:

$$
\begin{aligned}
I_{1} & =\int_{0}^{L_{2}} \bar{U}_{0}\left[(u \widetilde{u})+(v \widetilde{v})+N^{-2}(\psi \widetilde{\psi})\right]\left(L_{1}, y\right) d y \\
& -\int_{0}^{L_{2}} \lambda^{-1}(\psi \widetilde{u}+u \widetilde{\psi})\left(L_{1}, y\right) d y \\
& -\int_{0}^{L_{1}} \lambda^{-1}\left[(v \widetilde{\psi})\left(L_{2}, y\right)-(v \widetilde{\psi})(0, y)\right] d y .
\end{aligned}
$$

According to Rudin $(1991), D\left(A^{*}\right)$ consists of the $\widetilde{U}$ in $H$ such that $U \longrightarrow(A U, \widetilde{U})_{H}$ is continuous on $D(A)$ for the topology (norm) of $H$. If $U$ is restricted to the class of $\mathcal{C}^{\infty}$ functions with compact support in $\mathcal{M}^{\prime}$ (endowed with the norm of $H$ ), then $I_{1}=0$ and $U \longrightarrow I_{0}$ can only be continuous if $\mathcal{A}^{*} \widetilde{U}$ as defined in (4.44) belongs to $L^{2}\left(\mathcal{M}^{\prime}\right)^{3}$. If $\widetilde{U}$ belongs to $H$ and $\mathcal{A}^{*} \widetilde{U}$ belongs to $L^{2}\left(\mathcal{M}^{\prime}\right)^{3}$, then we already observed that Theorem 4.1 applies to $\mathcal{A}^{*}$ as well. Consequently the traces of $\widetilde{U}$ are defined as in Theorem 4.1 and the calculations in (4.52) are now valid for any such $\widetilde{U}$ (and $U$ in $D(A)$ not necessarily smooth). We now restrict $U$ to the class of $\mathcal{C}^{\infty}$ function on $\overline{\mathcal{M}}^{\prime}$ which belong to $D(A)$. Then the expressions above of $I_{0}$ and $I_{1}$ show that $U \longrightarrow(A U, \widetilde{U})_{H}$ can only be continuous in $U$ for the topology (norm) of $H$ if the following boundary conditions are satisfied:

$$
\left\{\begin{array}{l}
\widetilde{u}, \widetilde{v} \text { and } \widetilde{\psi}=0 \text { at } x=L_{1}, \\
\text { and } \widetilde{\psi}=0 \text { at } y=0 \text { and } L_{2} .
\end{array}\right.
$$

Conversely if $\widetilde{U} \in H, \mathcal{A}^{*} \widetilde{U} \in L^{2}\left(\mathcal{M}^{\prime}\right)^{3}$ and the conditions (4.54) are satisfied, then the calculations (4.52) are valid, $I_{1}=0$, and $U \longrightarrow$ $(A U, \widetilde{U})_{H}$ is continuous on $D(A)$ for the norm of $H$. Hence $\widetilde{U} \in D\left(A^{*}\right)$ and we conclude ${ }^{21}$ that

$$
D\left(A^{*}\right)=\left\{\widetilde{U} \in L^{2}\left(\mathcal{M}^{\prime}\right)^{3}, \mathcal{A}^{*} \widetilde{U} \in L^{2}\left(\mathcal{M}^{\prime}\right)^{3} \text {, and } \widetilde{U} \text { satisfies }(4.54)\right\}
$$

and that $A^{*} \widetilde{U}=\mathcal{A}^{*} \widetilde{U}$ for $\widetilde{U}$ in $D\left(A^{*}\right), \mathcal{A}^{*}$ as in (4.44).

Positivity of $A$ and $A^{*}$

The proof of the positivity is not done as in the subcritical case, since the regularity result of Theorem 4.4 is not available in this case. Instead, for $A$, to prove that $(A U, U)_{H} \geq 0$, for $U$ in $D(A)$, we will

\footnotetext{
${ }^{21}$ Remember that $A, A^{*}$ depend on $n$ through $\lambda=\lambda_{n}$; we write $A_{n}, A_{n}^{*}$ when the dependance on $n$ needs to be emphasized.
} 
construct a sequence of smooth functions $U_{n} \in D(A)$ such that, as $n \longrightarrow \infty$,

$$
\begin{aligned}
& U_{n} \longrightarrow U \text { in } H \text { strongly, } \\
& A U_{n} \longrightarrow A U \text { in } H \text { weakly. }
\end{aligned}
$$

Then $\left(A U_{n}, U_{n}\right)_{H} \longrightarrow(A U, U)_{H}$ and since $\left(A U_{n}, U_{n}\right)_{H} \geq 0$ by (4.51), $(A U, U)_{H} \geq 0$ follows. The proof for $A^{*}$ would be similar.

Given $U \in D(A)$, with $F=\left(f_{1}, f_{2}, f_{3}\right)=A U \in H$, we observe that the calculations (4.31)-(4.33) are still valid but now, since $\lambda>N / \widetilde{U}_{0}$ equation (4.33 is hyperbolic. In fact we are now going to treat (4.33) as a second order evolution equation in $x$ (wave equation), in which $x$ is the time-like variable and $y$ is the spatial variable. For such a wave equation we need to prescribe $\psi$ and $\psi_{x}$ at $x=0$, and $\psi$ at $y=0$ and $L_{2}$. These values of $\psi$ are given equal to 0 , and we are missing $\psi_{x}$ which we infer from the first and third equations (4.31) when $U$ is smooth, which we assume for the moment. Indeed since $v=0$ at $x=0, v_{y}=0$ and these equations, restricted to $x=0$, become a system

$$
\begin{aligned}
& \bar{U}_{0} u_{x}-\lambda^{-1} \psi_{x}=f_{1}, \\
& \bar{U}_{0} \psi_{x}-N^{2} \lambda^{-1} u_{x}=f_{3},
\end{aligned}
$$

which allows us to compute $u_{x}$ and $\psi_{x}$ at $x=0$; hence for $\psi_{x}$ :

$$
\psi_{x}(0, y)=\frac{1}{\bar{U}_{0}^{2}-N^{2} \lambda^{-2}}\left(\frac{N^{2}}{\lambda} f_{1}\left(L_{1}, y\right)+\bar{U}_{0} f_{3}\left(L_{1}, y\right)\right), 0<y<L_{2} .
$$

We continue to assume that all functions $\left(f_{1}, f_{2}, f_{3}, u, v, \psi\right)$ are sufficiently regular and we integrate (4.33) from 0 to $x$. Setting

$$
\Psi(x, y)=\int_{0}^{x} \psi\left(x^{\prime}, y\right) d y
$$

we obtain:

$$
\begin{aligned}
& -\left(\bar{U}_{0}^{2}-\frac{N^{2}}{\lambda^{2}}\right)\left(\psi_{x}(x, y)-\psi_{x}(0, y)\right) \\
& -\frac{N^{2}}{\lambda^{2}} \Psi_{y y}(x, y)=\frac{N^{2}}{\lambda}\left(f_{1}(x, y)-f_{1}(0, y)\right) \\
& +\frac{N^{2}}{\lambda} F_{2 y}(x, y)+\bar{U}_{0}\left(f_{3}(x, y)-f_{3}(0, y)\right)
\end{aligned}
$$

where

$$
F_{i}(x, y)=\int_{0}^{x} f_{i}\left(x^{\prime}, y\right) d x^{\prime}
$$


Taking (4.56) into account, there remains

$$
\begin{aligned}
& \left(\bar{U}_{0}^{2}-\frac{N^{2}}{\lambda^{2}}\right) \Psi_{x x}-\frac{N^{2}}{\lambda^{2}} \Psi_{y y}= \\
& =\frac{N^{2}}{\lambda} f_{1}+\frac{N^{2}}{\lambda} F_{2 y}+\bar{U}_{0} f_{3},
\end{aligned}
$$

which we aim to consider for $x>0$, with the "initial" and boundary conditions:

$$
\left\{\begin{array}{l}
\Psi=0 \text { and } \Psi_{x}=\psi=0 \text { at } x=0 \\
\Psi=0 \text { at } y=0 \text { and } L_{2} .
\end{array}\right.
$$

We obtain a priori estimates for $\Psi$ in a standard way by multiplying (4.59) by $\Psi_{x}$, integrating in $y$ and integrating by parts. We find

$$
\begin{aligned}
& \frac{1}{2}\left(\bar{U}_{0}^{2}-\frac{N^{2}}{\lambda^{2}}\right) \frac{d}{d x} \int_{0}^{L_{2}} \Psi_{x}^{2}(x, y) d y+\frac{N^{2}}{2 \lambda^{2}} \frac{d}{d x} \int_{0}^{L_{2}} \Psi_{y}^{2}(x, y) d y \\
& =-\int_{0}^{L_{2}}\left[\left(\frac{N^{2}}{\lambda} f_{1}-\frac{N^{2}}{\lambda} F_{2 y}+\bar{U}_{0} f_{3}\right) \Psi_{x}\right](x, y) d y .
\end{aligned}
$$

We then integrate in $x$ from 0 to $x$ to obtain, using (4.60):

$$
\begin{aligned}
& \frac{1}{2}\left(\bar{U}_{0}^{2}-\frac{N^{2}}{\lambda^{2}}\right) \int_{0}^{L_{2}} \Psi_{x}^{2}(x, y) d y+\frac{N^{2}}{2 \lambda^{2}} \int_{0}^{L_{2}} \Psi_{y}^{2}(x, y) d y \\
& =-\int_{0}^{x} \int_{0}^{L 2}\left[\left(\frac{N^{2}}{\lambda} f_{1}-\frac{N^{2}}{\lambda} F_{2 y}+\bar{U}_{0} f_{3}\right) \Psi_{x}\right]\left(x^{\prime}, y\right) d x^{\prime} d y
\end{aligned}
$$

The term involving $F_{2 y}$ can be integrated by parts, using (4.60); we find, all functions being sufficiently regular:

$$
\begin{aligned}
\frac{N^{2}}{\lambda} & \int_{0}^{x} \int_{0}^{L_{2}}\left(F_{2 y} \Psi_{x}\right)\left(x^{\prime}, y\right) d x^{\prime} d y \\
& =\frac{N^{2}}{\lambda} \int_{0}^{x} \int_{0}^{L_{2}}\left(F_{2} \Psi_{x y}\right)\left(x^{\prime}, y\right) d x^{\prime} d y \\
& =\frac{N^{2}}{\lambda} \int_{0}^{L_{2}}\left(F_{2} \Psi_{y}\right)(x, y) d y-\frac{N^{2}}{\lambda} \int_{0}^{x} \int_{0}^{L_{2}}\left(F_{2 x} \Psi_{y}\right)\left(x^{\prime}, y\right) d x^{\prime} d y \\
& =\frac{N^{2}}{\lambda} \int_{0}^{L_{2}} \Psi_{y}(x, y) \int_{0}^{x} f_{2}\left(x^{\prime}, y\right) d x^{\prime} d y-\frac{N^{2}}{\lambda} \int_{0}^{x} \int_{0}^{L_{2}}\left(f_{2} \Psi_{y}\right)\left(x^{\prime}, y\right) d x^{\prime} d y
\end{aligned}
$$


We insert this expression in (4.61) and integrate (4.61) in $x$ from 0 to $L_{1}$, which leads to:

$$
\begin{aligned}
& \left(\bar{U}_{0}^{2}-\frac{N^{2}}{\lambda^{2}}\right) \int_{\mathcal{M}^{\prime}} \Psi_{x}^{2}(x, y) d x d y+\frac{N^{2}}{\lambda^{2}} \int_{\mathcal{M}^{\prime}} \Psi_{y}^{2}(x, y) d x d y \\
& =\frac{2 N^{2}}{\lambda} \int_{\mathcal{M}^{\prime}} \Psi_{y}(x, y)\left(\int_{0}^{x} f_{2}\left(x^{\prime}, y\right) d x^{\prime}\right) d y d x \\
& -2 \int_{\mathcal{M}^{\prime}}\left[\frac{N^{2}}{\lambda} f_{1} \Psi_{x}+\frac{N^{2}}{\lambda}\left(\int_{0}^{x} f_{2}\left(x^{\prime}, y\right) d x^{\prime}\right) \Psi_{y}+\bar{U}_{0} f_{3} \Psi_{x}\right](x, y) d x d y .
\end{aligned}
$$

Since $\bar{U}_{0}>N / \lambda$, we easily deduce from (4.62) an estimate

$$
\begin{aligned}
& \int_{\mathcal{M}^{\prime}}\left(\Psi_{x}^{2}+\Psi_{y}^{2}\right)(x, y) d x d y \\
& \leq \kappa_{1}\left(\left|f_{1}\right|_{L^{2}\left(\mathcal{M}^{\prime}\right)}^{2}+\left|f_{2}\right|_{L^{2}\left(\mathcal{M}^{\prime}\right)}^{2}+\left|f_{3}\right|_{L^{2}\left(\mathcal{M}^{\prime}\right)}^{2}\right),
\end{aligned}
$$

where $\kappa_{1}$ depends only on the data, namely, $L_{1}, L_{2}, \bar{U}_{0}, N$ and $\lambda$. Alternatively (4.63) can be written as

$$
\int_{\mathcal{M}^{\prime}}\left(\psi^{2}+\Psi_{y}^{2}\right)(x, y) d x d y \leq \kappa_{1}|F|_{L^{2}\left(\mathcal{M}^{\prime}\right)^{3}}^{2}
$$

The calculations above have been made under the assumption that $U \in D(A)$ (and $A U=F$ ) are sufficiently regular. The lemma below extends (4.64) to all $U$ in $D(A)$.

Lemma 4.1. In the supercritical case (i.e. assuming (4.47)), (4.64) is valid for every $U=(u, v, \psi)$ in $D(A)$. There also exists a constant $\kappa_{2}$ depending only on the data such that

$$
|U|_{H} \leq \kappa_{2}|A U|_{H}, \quad \forall U \in D(A) .
$$

Proof. Given $U$ in $D(A)$, then $A U=F=\left(f_{1}, f_{2}, f_{3}\right)$ belongs to $H=$ $L^{2}\left(\mathcal{M}^{\prime}\right)^{3}$ and it can be approximated in $L^{2}\left(\mathcal{M}^{\prime}\right)^{3}$ by a sequence of smooth functions $F_{m}=\left(f_{1 m}, f_{2 m}, f_{3 m}\right)$ which are $\mathcal{C}^{\infty}$ with compact support in $\mathcal{M}^{\prime}$. With these $F_{m}$, we solve equation (4.59) with boundary and initial conditions (4.60) so that we obtain the $\Psi_{m}$ which satisfy (4.64).

As $n \rightarrow \infty$, the $F_{m}$ converge to $F$ in $L^{2}\left(\mathcal{M}^{\prime}\right)^{3}$ and the $\Psi_{m}$ converge to $\bar{\Psi}$ weakly in $H^{1}\left(\mathcal{M}^{\prime}\right)$, where $\bar{\Psi}$ is the (unique) solution of (4.59), (4.60) in $H^{1}\left(\mathcal{M}^{\prime}\right)$. We then define $\bar{\psi}=\partial \bar{\Psi} / \partial x$ which satisfies equation (4.33) in the distributional sense and (4.64) is satisfied by $\bar{\psi}, \bar{\Psi}$ and $F$. By inspection of (4.59), we notice that $\bar{\Psi}_{y y}$ and $F_{2 y}$ belong to $L_{x}^{2}\left(0, L_{1} ; H_{y}^{-1}\left(0, L_{2}\right)\right)$ so that

$$
\bar{\psi}_{x}=\bar{\Psi}_{x x} \in L_{x}^{2}\left(0, L_{1} ; H_{y}^{-1}\left(0, L_{2}\right)\right)
$$


and $\bar{\psi} \in \mathcal{C}_{x}\left(\left[0, L_{1}\right] ; H_{y}^{-1}\left(0, L_{2}\right)\right)$. Hence $\bar{\psi}(0, \cdot)$ is defined and it vanishes according to (4.60).

Now, integrating in $x$ the first and second equations (4.31) and imposing $\bar{u}=\bar{v}=0$ at $x=0$, we define $\bar{u}$ and $\bar{v}$ by setting

$$
\begin{aligned}
& \bar{U}_{0} \bar{u}-\frac{1}{\lambda} \bar{\psi}=\int_{0}^{x} f_{1} d x^{\prime}, \\
& \bar{U}_{0} \bar{v}-\frac{1}{\lambda} \bar{\psi}_{y} d x^{\prime}=\int_{0}^{x} f_{2} d x^{\prime} .
\end{aligned}
$$

We want to show that the third equation (4.31) is satisfied as well: differentiating the first equation (4.66) in $x$ and the second equation (4.66) in $y$ we find:

$$
\bar{U}_{0} \bar{u}_{x}-\frac{1}{\lambda} \bar{\psi}_{x}=f_{1}, \quad \bar{U}_{0} \bar{v}_{y}-\frac{1}{\lambda} \bar{\Psi}_{y y}=F_{2 y},
$$

and then

$$
\begin{aligned}
\bar{U}_{0} \bar{\psi}_{x} & -\frac{N^{2}}{\lambda}\left(\bar{u}_{x}+\bar{v}_{y}\right)= \\
& =\bar{U}_{0} \bar{\psi}_{x}-\frac{N^{2}}{\lambda \bar{U}_{0}}\left(\frac{1}{\lambda} \bar{\psi}_{x}+f_{1}+\frac{1}{\lambda} \bar{\Psi}_{y y}+F_{2 y}\right) \\
& =\left(\bar{U}_{0}-\frac{N^{2}}{\lambda^{2} \bar{U}_{0}}\right) \bar{\psi}_{x}-\frac{N^{2}}{\lambda^{2} \bar{U}_{0}} \bar{\Psi}_{y y}-\frac{N^{2}}{\lambda \bar{U}_{0}}\left(f_{1}+F_{2 y}\right) \\
& =(\text { by }(4.59)) \\
& =f_{3},
\end{aligned}
$$

so that all three equations (4.31) are satisfied by $\bar{U}$. Furthermore $\bar{U}$ satisfies the boundary conditions (4.60) and we conclude that $\bar{U} \in$ $D(A)$ and $A \bar{U}=F$. Since $A U=F$ as well, we will conclude that $\bar{U}=U$ by showing that $A$ is one-to-one.

To show that $A$ is one-to-one, consider $\widetilde{U} \in D(A)$ such that $A \widetilde{U}=0$. Then $\widetilde{\Psi}$ defined by (4.57) satisfies (4.59) and (4.60). At this point we do not know that $\widetilde{\Psi} \in H^{1}\left(\mathcal{M}^{\prime}\right)$, but, at least, we infer from (4.57) that $\widetilde{\Psi} \in L^{2}\left(\mathcal{M}^{\prime}\right)$ since $\widetilde{\psi} \in L^{2}\left(\mathcal{M}^{\prime}\right)$. We then infer from Lions and Magenes (1972) that (4.59) - (4.60) has a unique solution in $L^{2}\left(\mathcal{M}^{\prime}\right)$, so that $\widetilde{\Psi}=0$. From this we conclude that $\widetilde{\psi}=0$ and $\widetilde{u}$ and $\widetilde{v}$ also vanish since they satisfy equations (4.66), because of the boundary conditions at $x=0$. Hence $\widetilde{U}=0$ and $A$ is one-to-one.

Returning to $U$, we conclude at this point that $\psi$ and $\Psi$ satisfy (4.64) which was the first statement in this lemma. 
There remains to prove $(4.65) ;|\psi|_{L^{2}\left(\mathcal{M}^{\prime}\right)} \leq \kappa|A U|_{H}$ follows from (4.64), and the similar results for $u$ and $v$ follow from (4.66) (and $(4.64))$.

The proof of the Lemma is complete.

We can now prove $(4.50) .^{22}$

Theorem 4.7. In the supercritical case (i.e. assuming (4.47)), for every $U \in D\left(A_{n}\right), A_{n}$ defined in (4.49), we have $\left(A_{n} U, U\right)_{L^{2}\left(\mathcal{M}^{\prime}\right)^{3}} \geq 0$.

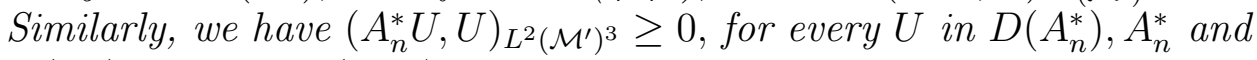
$D\left(A_{n}^{*}\right)$ defined in (4.55).

Proof. We prove the result for $A$, the proof would be similar for $A^{*}$.

Considering $U \in D(A)$, we approximate $A U=F$ by a sequence of smooth functions $F_{m}$ as in Lemma 4.1. To each function $F_{m}$, we associate $U_{m} \in D(A)$ such that $A U_{m}=F_{m}$ : each $U_{m}$ is constructed exactly as we constructed $\bar{U}$ in Lemma 4.1 , and $U_{m}$ is smooth. We easily check that, as $m \rightarrow \infty, U_{m}$ weakly converges in $H$ to $U$, whereas $A U_{m}=F_{m}$ strongly converges in $H$ to $A U=F$. Hence

$$
\left(A U_{m}, U_{m}\right)_{H} \longrightarrow(A U, U)_{H},
$$

and since $\left(A U_{m}, U_{m}\right)_{H} \geq 0$ by (4.51), $U_{m}$ being sufficiently regular, we conclude that $(A U, U)_{H} \geq 0$.

Remark 4.8. As indicated in Remark 4.6, and based on the previous results, we can show for each $n>n_{c}$ that $-A=-A_{n}$ is the infinitesimal generator of a contraction semi-group. Then by application of the Hille-Yoshida theorem we can solve the initial and boundary value problem associated with equations (4.7), for each such $n$. We refrain from developing this and we will study all subcritical and supercritical modes at once (together) in the next section.

\subsection{The initial and boundary value problem for the full sys-}

tem. In this section we aim to combine the results of the previous sections and to investigate the well-posedness for equations (4.3) associated with the suitable initial and boundary conditions. We successively consider the case of homogeneous and nonhomogeneous boundary conditions.

\footnotetext{
${ }^{22}$ We recall that $A$ and $A^{*}$ depend on $n$ as $\lambda=\lambda_{n}$.
} 
4.4.1. The homogeneous boundary condition case. As explained in (4.9) the function $U$ and its respective components are decomposed in the form $U=U^{0}+U^{I}+U^{I I}$. Accordingly the basic function space $H$ will be $L^{2}(\mathcal{M})^{3}$ or

$$
H=H^{0} \times \dot{L}^{2}(\mathcal{M})^{3},
$$

where $H^{0}$ is the same as $H_{0}$ in $(4.23)$, and $\dot{L}^{2}(\mathcal{M})$ consists of the orthogonal, in $L^{2}(\mathcal{M})$, of the space of functions independent of $z$. Like in Section 4.1.5 the elements of $H^{0}$ will be the vectors $\mathbf{u}^{0}=\left(u^{0}, v^{0}\right)$. The elements of $\dot{L}^{2}(\mathcal{M})^{3}$ will be the triplets $U=(u, v, \psi)$; each of these functions possesses an expansion of the form (1.19) from which we can accordingly identify the functions with the product of their components, and the space $L^{2}(\mathcal{M})$ with the product of an infinite sequence of spaces $L^{2}\left(\mathcal{M}^{\prime}\right)$. The space $H$ is a subspace of $L^{2}(\mathcal{M})^{3}$, just remembering that $\psi_{0}=0$, and its natural scalar product and norms are essentially those of $L^{2}(\mathcal{M})^{3}$, more precisely,

$$
\begin{aligned}
(U, \widetilde{U})_{H} & =((u, v, \psi),(\widetilde{u}, \widetilde{v}, \widetilde{\psi}))_{L^{2}(\mathcal{M})^{3}} \\
& =(u, \widetilde{u})_{L^{2}(\mathcal{M})}+(v, \widetilde{v})_{L^{2}(\mathcal{M})}+\frac{1}{N^{2}}(\psi, \widetilde{\psi})_{L^{2}(\mathcal{M})}, \\
|U|_{H} & =\left[(U, U)_{H}\right]^{1 / 2} .
\end{aligned}
$$

Each $U$ can be seen as the sum of its three components

$$
U=U^{0}+U^{I}+U^{I I}
$$

or it can be identified with the infinite sequence of its components $\left\{U_{n}\right\}_{n \geq 0}$, in which case $^{23}$

$$
|U|_{H}^{2}=\left|\mathbf{u}^{0}\right|_{L^{2}\left(\mathcal{M}^{\prime}\right)^{2}}^{2}+\Sigma_{n=1}^{\infty}\left|U_{n}\right|_{L^{2}(\mathcal{M})^{2}}^{2}
$$

The semigroup

We now introduce the operator $A$ and its domain $D(A)$ in $H$. We have $D(A)=D\left(A^{0}\right) \times D\left(A^{I}\right) \times D\left(A^{I I}\right)$, where the space $D\left(A^{0}\right)$ is the same as in (4.26),

$$
D\left(A^{0}\right)=\left\{\mathbf{u}^{0} \in H^{0}, \mathbf{u}_{x}^{0} \in L^{2}\left(\mathcal{M}^{\prime}\right)^{2}\right\} .
$$

Then (compare to $(4.30))$ :

\footnotetext{
${ }^{23}$ Remember that $\psi^{0}=0$ so that $U^{0}=\mathbf{u}^{0}=\left(u^{0}, v^{0}\right)$.
} 


$$
\begin{gathered}
D\left(A^{I}\right)=\left\{U^{I}=\left(U_{1}, \ldots, U_{n_{c}}\right), U_{n} \in L^{2}\left(\mathcal{M}^{\prime}\right)^{3}, \mathcal{A}_{n} U_{n} \in L^{2}\left(\mathcal{M}^{\prime}\right)^{3},\right. \\
\left.n=1, \ldots, n_{c}, U^{I} \text { satisfies },(4.70)\right\}, \\
70) \quad\left\{\begin{array}{l}
\psi^{I}=0 \text { at } x=L_{1}, \text { and } y=0, L_{2}, \\
v^{I}=0 \text { and } u^{I}+\phi^{I} / \bar{U}_{0}=0 \text { at } x=0 .
\end{array}\right.
\end{gathered}
$$

Here we introduced for convenience the function $\phi=\psi^{0}+\phi^{I}+\phi^{I I}=$ $\left\{\phi_{n}\right\}_{n \geq 0}$, with, according to (4.5),

$$
\phi_{n}=-\frac{1}{\lambda_{n}} \psi_{n}, \quad n \geq 1 \text {. }
$$

Finally (compare to $(4.49))$ :

$$
\begin{aligned}
& D\left(A^{I I}\right)=\left\{U^{I I}=\left\{U_{n}\right\}_{n>n_{c}}, U_{n} \in L^{2}\left(\mathcal{M}^{\prime}\right)^{3}, \mathcal{A}_{n} U_{n}\right. \\
& \left.\in L^{2}\left(\mathcal{M}^{\prime}\right)^{3}, n=1, \ldots, n_{c}, U^{I I} \text { satisfies }(4.73)\right\}, \\
& \left\{\begin{array}{l}
u^{I I}=v^{I I}=\psi^{I I}=0 \text { at } x=0, \\
\psi^{I I}=0 \text { at } y=0 \text { and } L_{2} .
\end{array}\right.
\end{aligned}
$$

For $U=\left(\mathbf{u}_{0}, U^{I}, U^{I I}\right)$ in $D(A)$, we set $A U=\left(A^{0} \mathbf{u}^{0}, A^{I} U^{I}, A^{I I} U^{I I}\right)$ where

$$
A^{0} \mathbf{u}^{0}=P_{H^{0}}\left(\bar{U}_{0} \frac{\partial \mathbf{u}^{0}}{\partial x}+f e_{z} \wedge \mathbf{u}^{0}\right)
$$

as in (4.25) and we define $A^{I} U^{I}$ and $A^{I I} U^{I I}$ componentwise by setting

$$
A_{n} U_{n}=\mathcal{A}_{n} U_{n}, \quad \text { for } 1 \leq n,
$$

$\mathcal{A}_{n}$ as in (4.21) with $\lambda=\lambda_{n}$.

We now need to define the adjoint $A^{*}$ of $A$ and prove that $A$ and $A^{*}$ are positive which will follow promptly from the results in the previous sections.

For the adjoint, it is easy to see that

$$
D\left(A^{*}\right)=D\left(A^{0 *}\right) \times D\left(A^{I *}\right) \times D\left(A^{I I * *}\right),
$$

with $D\left(A^{0 *}\right)=D\left(A^{0}\right)$ as shown in Section 4.1.5, $D\left(A^{I *}\right)$ defined in (4.46) and $D\left(A^{I *}\right)$ defined in (4.55). Indeed, according to Rudin 
(1991), $\widetilde{U} \in D\left(A^{*}\right)$ if and only if,

$$
U \rightarrow(A U, \widetilde{U})_{H}=\left(A^{0} \mathbf{u}^{0}, \widetilde{\mathbf{u}}^{0}\right)+\left(A^{I} U^{I}, \widetilde{U}^{I}\right)+\left(A^{I I} U^{I I}, \widetilde{U}^{I I}\right),
$$

is continuous on $D(A)$ for the topology (norm) of $H$. Considering successively $U=\left(\mathbf{u}^{0}, 0,0\right), U=\left(0, U^{I}, 0\right)$, and $U=\left(0,0, U^{I I}\right)$, we obtain that $D(A)$ is included in the space in the right-hand side of (4.74). Conversely any $\widetilde{U}$ in the right-hand side of (4.74) belongs to $D(A)$ and hence (4.74) is proven.

We can prove the following

Theorem 4.9. The operator $-A$ is infinitesimal generator of a semigroup of contractions in $H$.

Proof. According to Yosida (1980) and Hille and Phillips (1974), it suffices to show that

i) $A$ and $A^{*}$ are closed operators, and their domains $D(A)$ and $D\left(A^{*}\right)$ are dense in $H$.

ii) $A$ and $A^{*}$ are positive:

$$
\begin{array}{ll}
(A U, U)_{H} \geq 0, & \forall U \in D(A), \\
\left(A^{*} U, U\right)_{H} \geq 0, & \forall U \in D\left(A^{*}\right) .
\end{array}
$$

For i) we observe, as is well-known, that $D\left(A^{*}\right)$ (resp. $D(A)$ ) dense in $H$ implies that $A$ (resp. $A^{*}$ ) is closed. We proceed componentwise for, say, $D(A): D\left(A^{0}\right)$ defined in (4.68) is dense in $H^{0}$, since the $\mathcal{C}^{\infty}$ functions $\mathbf{u}^{0}=\left(u^{0}, v^{0}\right)$ with compact support in $\mathcal{M}^{\prime}$ and such that $\operatorname{div} \mathbf{u}^{0}=u_{x}^{0}+v_{y}^{0}=0$ are dense in $H^{0}$; see e.g. Temam (2001); and for $D\left(A^{I}\right)$ and $D\left(A^{I I}\right)$ we simply observe that the $\mathcal{C}^{\infty}$ functions with compact support in $\mathcal{M}^{\prime}$ are dense in $L^{2}\left(\mathcal{M}^{\prime}\right)$.

Finally for (4.75) we proceed componentwise and use the results of the previous sections, e.g. for $A$ :

$$
(A U, U)_{H}=\left(A^{0} \mathbf{u}^{0}, \mathbf{u}^{0}\right)_{H^{0}}+\left(A^{I} U^{I}, U^{I}\right)_{H^{I}}+\left(A^{I I} U^{I I}, U^{I I}\right)_{H^{I I}} .
$$

The first term in the right-hand side of (4.76) has been shown to be positive $(=0$ in fact, see (4.28)). The second term is equal to

$$
\sum_{n=1}^{n c}\left(A_{n} U_{n}, U_{n}\right)_{L^{2}\left(\mathcal{M}^{\prime}\right)^{3}},
$$

and each of these terms is positive as shown in (4.39). Finally the third term

$$
\left(A^{I I} U^{I I}, U^{I I}\right)_{H^{I I}}=\sum_{n>n_{c}}\left(A_{n} U_{n}, U_{n}\right)_{L^{2}\left(\Gamma_{i}\right)^{3}},
$$


and each term of the series is positive according to (4.50).

The initial and boundary value problems

We now consider the whole system of three-dimensional linearized Primitive Equations, namely (4.3) and introduce the initial and boundary conditions. We start with the homogeneous boundary conditions and treat subsequently the case of nonhomogeneous boundary conditions.

As implied by the previous sections the boundary conditions will be different for the subcritical and supercritical components of $U=$ $(u, v, \psi)=\left(U^{0}, U^{I}, U^{I I}\right)$. Hence for $U^{0}=\mathbf{u}^{0}\left(\psi^{0}=0\right)$, we set (see $(4.26))$ :

$$
\mathbf{u}^{0} \cdot \mathbf{n}=0 \text { on } \partial \mathcal{M}^{\prime}
$$

For $U^{I}$, according to (4.29), the boundary conditions read

$$
\left\{\begin{array}{l}
\psi^{I}=0 \text { at } x=L_{1}, \text { and } y=0, L_{2}, \\
v^{I}=0 \text { and } u_{n}=\psi_{n} / \lambda_{n} \bar{U}_{0} \text { at } x=0, n=1, \ldots, n_{c} .
\end{array}\right.
$$

For $U^{I I}$ the boundary conditions are inferred from (4.49) and read

$$
\left\{\begin{array}{l}
u^{I I}=v^{I I}=\psi^{I I}=0 \text { at } x=0, \\
\text { and } \psi^{I I}=0 \text { at } y=0 \text { and } L_{2} .
\end{array}\right.
$$

All these boundary conditions are taken into account in the domain $D(A)$ of $A$. Finally if we add the initial conditions

$$
U(0)=(u(0), v(0), \psi(0))=U_{0}=\left(u_{0}, v_{0}, \psi_{0}\right),
$$

then the initial and boundary value problem consisting of equations (4.3), and (4.77) - (4.80) is equivalent to the abstract initial value problem

$$
\begin{gathered}
\frac{d U}{d t}+A U=F, \\
U(0)=U_{0} .
\end{gathered}
$$

Note that $F=\left(F_{u}, F_{v}, F_{w}\right)$ which does not appear in (4.3) is added here for mathematical generality and to study below the case of nonhomogeneous boundary conditions. By Theorem 4.9 this problem is now solved by the Hille-Yoshida theorem and we have 
Theorem 4.10. Let $H, A$ and $D(A)$ be defined as in (4.67) - (4.73). Then the initial value problem (4.81) - (4.82) is well-posed. That is, for every $U_{0} \in D(A)$, and $F \in L^{1}(0, T, H)$, with $F^{\prime}=d F / d t$ in $L^{1}(0, T ; H),(4.81)$ - (4.82) has a unique solution $U$ such that

$$
U \in \mathcal{C}([0, T] ; H) \cap L^{\infty}(0, T ; D(A)), \quad \frac{d U}{d t} \in L^{\infty}(0, T ; H) .
$$

4.4.2. The nonhomogeneous boundary conditions. We now turn to the case of nonhomogeneous boundary conditions for (4.77) - (4.79), that is we want to solve (4.3) with (4.77) - (4.79) in which the boundary conditions are now nonhomogeneous, and with initial condition (4.80). We assume that all boundary data are inferred from a function $U^{g}=$ $\left(\mathbf{u}^{g 0}, U^{g I}, U^{g I I}\right)$ which is defined in $\mathcal{M} \times[0, T]$. We also assume that $U^{g}$ is given by its normal modes expansion:

$$
\begin{array}{r}
U^{g}(x, y, z, t)=\left(\sum_{n \geq 0} u_{n}^{g}(x, y, t) \mathcal{U}_{n}(z), \sum_{n \geq 0} v_{n}^{g}(x, y, t) \mathcal{U}_{n}(z)\right. \\
\left.\sum_{n \geq 1} \psi_{n}^{g}(x, y, t) \mathcal{W}_{n}(z)\right)
\end{array}
$$

We now set

$$
U=U^{\#}+U^{g},
$$

and observe that $U^{\#} \in D(A)$ if $U^{\#}$ is smooth enough (homogeneous boundary conditions). Then $U^{\#}$ will be sought as the solution of the linear evolution equation

$$
\begin{aligned}
& \frac{d U^{\#}}{d t}+A U^{\#}=F^{\#}, \\
& U^{\#}(0)=U_{0}^{\#},
\end{aligned}
$$

where

$$
U_{0}^{\#}=U_{0}-\left.U^{g}\right|_{t=0}
$$

and

$$
F^{\#}=F-\frac{\partial U^{g}}{\partial t}-\mathcal{A} U^{g} .
$$

Here $\mathcal{A} U^{g}$ is defined by its normal mode expansion, where each $\left(\mathcal{A} U^{g}\right)_{n}$ is equal to $\mathcal{A}_{n} U_{n}^{g}, \mathcal{A}_{n}$ as in (4.21).

Theorem 4.10 will be applicable to (4.85) and we will obtain the desired existence and uniqueness result for $U$, provided we assume that $U_{0}^{\#}$ and $F^{\#}$ satisfy the hypotheses of Theorem 4.10 . It is very easy to 
give sufficient (non necessarily optimal) conditions on $U^{g}$ which guarantee that $U_{0}^{\#} \in D(A)$ and $F^{\#}$ and $d F^{\#} / d t$ are in $L^{1}\left(0, T ; \mathbf{L}^{2}(\mathcal{M})^{3}\right)$. We assume e.g. the following

$$
\begin{aligned}
& U_{0}, \frac{\partial U_{0}}{\partial x}, \frac{\partial U_{0}}{\partial y} \in L^{2}(\mathcal{M})^{3}, \text { and } \operatorname{div} \mathbf{u}_{0}^{0}=0 \\
& F, \frac{\partial F}{\partial t} \in L^{1}\left(0, T ; L^{2}(\mathcal{M})^{3}\right) \\
& U^{g}, \frac{\partial U^{g}}{\partial t}, \frac{\partial U^{g}}{\partial x}, \frac{\partial U^{g}}{\partial y}, \frac{\partial^{2} U^{g}}{\partial t^{2}}, \frac{\partial^{2} U^{g}}{\partial x \partial t}, \frac{\partial^{2} U^{g}}{\partial y \partial t} \in \mathcal{C}\left([0, T] ; L^{2}(\mathcal{M})^{3}\right) .
\end{aligned}
$$

In addition we require that $U_{0}$ and $U^{g}$ satisfy certain compatibility conditions, for $t=0$, and $(x, y) \in \partial \mathcal{M}^{\prime}$, conditions which guarantee that $U_{0}^{\#} \in D(A)$. Setting $U_{0}=\left(\widetilde{u}_{0}, \widetilde{v}_{0}, \widetilde{\psi}_{0}\right)=\left(\widetilde{U}_{0}^{0}, \widetilde{U}_{0}^{I}, \widetilde{U}_{0}^{I I}\right),{ }^{24}$ we require

$$
\begin{aligned}
& \widetilde{\mathbf{u}}^{0} \cdot \mathbf{n}=\mathbf{u}^{0 g} \cdot \mathbf{n}, \quad \text { on } \partial \mathcal{M}^{\prime}, \text { at } t=0 \\
& \widetilde{\psi}_{0}^{I}=\widetilde{\psi}^{g I} \text { at } t=0 \text { and } x=L_{1}, \text { or } y=0 \text { or } L_{2}, \\
& \widetilde{v}_{0}^{I}=\widetilde{v}^{g I} \text { and } \widetilde{u}_{o n}=\widetilde{\psi}_{o n} / \lambda_{n} \bar{U}_{0}=\widetilde{u}_{n}^{g}-\widetilde{\psi}_{n}^{g} / \lambda_{n} \bar{U}_{0} \\
& \quad \text { at } x=0 \text { and } t=0, n=1, \ldots, n_{c}, \\
& \widetilde{u}_{0}^{I I}-\widetilde{u}^{g I}=\widetilde{v}_{0}^{I I}-\widetilde{v}^{g I}=\widetilde{\psi}_{0}^{I I}-\widetilde{\psi}^{g I I}, \text { at } x=0 \text { and } t=0, \\
& \widetilde{\psi}_{0}^{I I}-\widetilde{\psi}^{g I I}=0, \text { at } t=0 \text { and } y=0 \text { or } L_{2} .
\end{aligned}
$$

With the regularity hypotheses (4.88) and the compatibility hypotheses (4.89), we obtain $U$ satisfying

$$
\begin{aligned}
& U \in \mathcal{C}\left([0, T] ; L^{2}(\mathcal{M})^{3}\right), \\
& \mathcal{A} U \in L^{\infty}\left(0, T ; L^{2}(\mathcal{M})^{3}\right), \\
& \frac{\partial U}{\partial t} \in L^{\infty}\left(0, T ; L^{2}(\mathcal{M})^{3}\right),
\end{aligned}
$$

\footnotetext{
${ }^{24}$ The tildes here on $u_{0}, v_{0}, \psi_{0}$, etc. are intended to distinguish these initial data from the zero modes of $U(t)$.
} 
and the boundary conditions for $0<t<T$ :

$$
\begin{aligned}
& \mathbf{u}^{0} \cdot \mathbf{n}=\mathbf{u}^{g} \cdot \mathbf{n} \text { on } \partial \mathcal{M}^{\prime},-L_{3}<z<0, \\
& \psi^{I}=\psi^{g I} \text { at } x=L_{1} \text { and } y=0, L_{2}, \\
& v^{I}=v^{g I} \text { at } x=0, \\
& u_{n}^{I}-\psi_{n}^{I} / \lambda_{n} \bar{U}_{0}=u_{n}^{g I}-\psi_{n}^{g I} / \lambda_{n} \bar{U}_{0}, \text { at } x=0, n=1, \ldots, n_{c}, \\
& u^{I I}=u^{g I I}, v^{I I}=v^{g I I}, \psi^{I I}=\psi^{g I I} \text { at } x=0, \\
& \psi^{I I}=\psi^{g I I} \text { at } y=0 \text { and } L_{2} .
\end{aligned}
$$

In summary, we have proven the following theorem:

Theorem 4.11. We assume that $U_{0}, F$ and $U^{g}$ are given satisfying the hypotheses (4.88) and (4.89). Then there exists a unique $U$ solution of the Primitive Equations (4.3), satisfying the regularity properties (4.90), the boundary condition (4.91) and the initial condition (4.82).

\section{Conclusion}

In this article we have analyzed the inviscid linearized primitive equations considering successively the dimensions two, two and half and three. In accordance with the previously known result that these equations cannot be well-posed for any set of local boundary conditions, we have proposed nonlocal boundary conditions and established their suitability.

In space dimension two, numerical simulations have been performed for both the linearized and nonlinear nonviscous primitive equations. The suitability of the boundary conditions that we have proposed is numerically confirmed in the linear case. In the nonlinear case the same boundary conditions have been used and have shown to be also numerically suitable. Furthermore the nonoccurence of numerical blow-up is an indication that these boundary conditions are appropriate in the nonlinear case as well

Future work in this domain will consist on the theoretical side in considering more complicated background flows in the linear case, and the full nonlinear equations. On the computational side the three-dimensional case and more involved equations (richer physics) should be investigated.

Acknowledgments. This work was partially supported by the National Science Foundation under the grant NSF-DMS-0604235, and by the Research Fund of Indiana University. 


\section{REFERENCES}

Bennett, A. F. and Chua, B. S. (1999). Open boundary conditions for Lagrangian geophysical fluid dynamics. J. Comput. Phys., 153(2), 418-436.

Bennett, A. F. and Kloeden, P. E. (1978). Boundary conditions for limited-area forecasts. J. Atmospheric Sci., 35(6), 990-996.

Bernardi, C. and Maday, Y. (1997). Spectral methods. In Handbook of numerical analysis, Vol. V, Handb. Numer. Anal., V, pages 209-485. North-Holland, Amsterdam.

Blayo, E. and Debreu, L. (2005). Revisiting open boundary conditions from the point of view of characteristic variables. Ocean Modelling, 9, 231-252.

Brézis, H. (1970). On some degenerate nonlinear parabolic equations. In Nonlinear Functional Analysis (Proc. Sympos. Pure Math., Vol. XVIII, Part 1, Chicago, Ill., 1968), pages 28-38. Amer. Math. Soc., Providence, R.I.

Brézis, H. (1973). Opérateurs maximaux monotones et semi-groupes de contractions dans les espaces de Hilbert. North-Holland Publishing Co., Amsterdam.

Burq, N. and Gérard, P. (2003). Contrôle optimal des équations aux dérivées partielles. Ecole Polytechnique, Palaiseau, France.

Cao, C. and Titi, E. (2007). Global well-posedness of the threedimensional viscous primitive equations of large scale ocean and atmosphere dynamics. Ann. of Math., 165(2). To appear.

Charney, J. G., Fjörtoft, R., and von Neumann, J. (1950). Numerical integration of the barotropic vorticity equation. Tellus, 2, 237-254.

Dumas, F. and Lazure, P. (2007). An external-internal mode coupling for a 3d hydrodynamical model for applications at regional scale (mars). Advances in Water Resources. . To appear.

Gottlieb, D. and Hesthaven, J. S. (2001). Spectral methods for hyperbolic problems. J. Comput. Appl. Math., 128(1-2), 83-131. Numerical analysis 2000, Vol. VII, Partial differential equations.

Grisvard, P. (1985). Elliptic problems in nonsmooth domains. Monographs and Studies in Mathematics. Pitman, Boston.

Henry, D. (1981). Geometric theory of semilinear parabolic equations, volume 840 of Lecture Notes in Mathematics. Springer-Verlag, Berlin.

Hille, E. and Phillips, R. (1974). Functional analysis and semi-groups. American Mathematical Society, Providence, R. I. Third printing of the revised edition of 1957, American Mathematical Society Colloquium Publications, Vol. XXXI. 
John, F. (1982). Partial Differential Equations. Springer-Verlag, NewYork. Fourth Edition.

Kobelkov, G. (2006). Existence of a solution 'in the large' for the 3D large-scale ocean dynamics equations. C. R. Math. Acad. Sci. Paris, 343(4), 283-286.

Kukavica, I. and Ziane, M. (2007). On the regularity of the primitive equations of the ocean. To appear.

Lions, J. (1965). Problèmes aux limites dans les équations aux dérivées partielles. Les Presses de l'Université de Montréal, Montreal, Que. Reedited in Lions (2003).

Lions, J. (2003). Selected work, Vol 1. EDS Sciences, Paris.

Lions, J., Temam, R., and Wang, S. (1992a). New formulations of the primitive equations of atmosphere and applications. Nonlinearity, 5(2), 237-288.

Lions, J., Temam, R., and Wang, S. (1992b). On the equations of the large-scale ocean. Nonlinearity, 5(5), 1007-1053.

Lions, J.-L. and Magenes, E. (1972). Non-homogeneous boundary value problems and applications. Springer-Verlag, New-York-Heidelberg. Vols. I \& II, Translated from the French by P. Kenneth.

Madec, G., Delecluse, P., Imbard, M., and Lévy, C. (1998). OPA 8.1. Ocean General Circulation Model Reference Manual. Institut Pierre Simon Laplace.

Oliger, J. and Sundström, A. (1978). Theoretical and practical aspects of some initial boundary value problems in fluid dynamics. SIAM J. Appl. Math., 35(3), 419-446.

Pazy, A. (1983). Semigroups of operators in Banach spaces. In Equadiff 82 (Würzburg, 1982), volume 1017 of Lecture Notes in Math., pages 508-524. Springer, Berlin.

Pedlosky, J. (1987). Geophysical fluid dynamics, 2nd edition. Springer. Petcu, M., Temam, R., and Ziane, M. (2008). Mathematical problems for the primitive equations with viscosity. In R. T. P.G. Ciarlet EDs and J. T. G. Eds, editors, Handbook of Numerical Analysis. Special Issue on Some Mathematical Problems in Geophysical Fluid Dynamics, Handb. Numer. Anal. Elsevier, New York.

Rousseau, A., Temam, R., and Tribbia, J. (2004). Boundary layers in an ocean related system. J. Sci. Comput., 21(3), 405-432.

Rousseau, A., Temam, R., and Tribbia, J. (2005a). Boundary conditions for an ocean related system with a small parameter. In Nonlinear PDEs and Related Analysis, volume 371, pages 231-263. Gui-Qiang Chen, George Gasper and Joseph J. Jerome Eds, Contemporary Mathematics, AMS, Providence. 
Rousseau, A., Temam, R., and Tribbia, J. (2005b). Boundary conditions for the 2D linearized PEs of the ocean in the absence of viscosity. Discrete Contin. Dyn. Syst., 13(5), 1257-1276.

Rousseau, A., Temam, R., and Tribbia, J. (2007). Numerical simulations of the inviscid primitive equations in a limited domain. In Analysis and Simulation of Fluid Dynamics, Advances in Mathematical Fluid Mechanics. Caterina Calgaro and Jean-François Coulombel and Thierry Goudon.

Rudin, W. (1991). Functional analysis. International Series in Pure and Applied Mathematics. McGraw-Hill Inc., New York, second edition. Salmon, R. (1998). Lectures on geophysical fluid dynamics. Oxford University Press, New York.

Temam, R. (2001). Navier-Stokes equations. AMS Chelsea Publishing, Providence, RI. Theory and numerical analysis, Reprint of the 1984 edition.

Temam, R. and Tribbia, J. (2003). Open boundary conditions for the primitive and Boussinesq equations. J. Atmospheric Sci., 60(21), 2647-2660.

Temam, R. and Ziane, M. (2004). Some mathematical problems in geophysical fluid dynamics. In S. Friedlander and D. Serre, editors, Handbook of mathematical fluid dynamics. North-Holland.

Warner, T., Peterson, R., and Treadon, R. (1997). A tutorial on lateral boundary conditions as a basic and potentially serious limitation to regional numerical weather prediction. Bull. Amer. Meteor. Soc., 78(11), 2599-2617.

Washington, W. and Parkinson, C. (1986). An introduction to threedimensional climate modelling. Oxford Univ. Press.

Yosida, K. (1980). Functional analysis. Springer-Verlag, Berlin, 6th edition. 